University of Nebraska - Lincoln

DigitalCommons@University of Nebraska - Lincoln

\title{
$5-2012$
}

\section{Regioselective Synthesis of Tetraalkynylarenes by Consecutive Dual Sonogashira Coupling Reactions of the Bis(triflate) of 4,5-Diiodobenzene-1,2-diol}

\author{
Thomas J. Fisher \\ University of Nebraska-Lincoln, TFisher@unlserve.unl.edu \\ Patrick H. Dussault \\ University of Nebraska-Lincoln, pdussault1@unl.edu
}

Follow this and additional works at: https://digitalcommons.unl.edu/chemistrydussault

Part of the Chemistry Commons

Fisher, Thomas J. and Dussault, Patrick H., "Regioselective Synthesis of Tetraalkynylarenes by Consecutive Dual Sonogashira Coupling Reactions of the Bis(triflate) of 4,5-Diiodobenzene-1,2-diol" (2012). Patrick Dussault Publications. 17.

https://digitalcommons.unl.edu/chemistrydussault/17

This Article is brought to you for free and open access by the Published Research - Department of Chemistry at DigitalCommons@University of Nebraska - Lincoln. It has been accepted for inclusion in Patrick Dussault Publications by an authorized administrator of DigitalCommons@University of Nebraska - Lincoln. 


\title{
Regioselective Synthesis of Tetraalkynylarenes by Consecutive Dual Sonogashira Coupling Reactions of the Bis(triflate) of 4,5-Diiodobenzene-1,2-diol
}

\author{
Thomas J. Fisher and Patrick H. Dussault \\ Department of Chemistry and Center for Nanohybrid Functional Materials, \\ University of Nebraska-Lincoln, Lincoln, NE 68588, USA \\ Corresponding author - P. Dussault, pdussault1@unl.edu
}

\begin{abstract}
The regioselective synthesis of nonsymmetric tetraalkynylarenes has been readily achieved through consecutive sets of Sonagashira cross-coupling reactions of the bis(triflate) derivative of 4,5-diiodobenzene-1,2-diol. The initial coupling reactions proceeded with nearly complete selectivity for the reaction at the Ar-I linkages. Subsequent coupling reactions at the Ar-OTf linkages were efficiently conducted. The tetraalkynylarene products are of interest as components of organic molecular materials.
\end{abstract}

Keywords: Cross-coupling, Regioselectivity, Palladium, Alkynes

\section{Introduction}

The palladium-catalyzed cross-coupling reactions of aryl halides or triflates with terminal alkynes is a popular method for the synthesis of arylalkynes. ${ }^{1}$ One class of reaction products, tetraalkynylarenes, is of particular interest in the field of organic materials. ${ }^{2}$ Tetraalkynylbenzenes containing four identical alkyne side-chains can be prepared from nonselective coupling reactions of tetrahaloarenes. ${ }^{3}$ However, regio-defined synthesis of unsymmetrical tetraalkynylarenes also requires control of the sequence of cross-coupling steps. A reported solution to this challenge exploits the greater reactivity of aryl iodides relative to aryl bromides. ${ }^{1,2}$ However, in some cases, the sequential coupling reactions of iodo/bromoarenes have resulted in low yields of tetraalkynylarenes, presumably reflecting the lower reactivity and cross-coupling efficiency of aryl bromides and/or the corresponding requirement for more forcing reaction conditions. ${ }^{4}$ Indeed, in the course of investigations requiring the preparation of unsymmetrical tetraalkynes, we found that attempted two-fold Sonagashira cross-coupling of model 1,2-dibromoarenes failed to give bis(alkyne) products. As a result, we became interested in the possibility of performing consecutive dual Sonogashira coupling reactions with an arene bearing both ortho-diiodide and ortho-bis(triflate) units.

The higher reactivity of aryl iodides relative to aryl triflates as substrates for C-C cross-coupling reactions is well established, ${ }^{5}$ and we were encouraged by a few reports describing the successful use of arene-1,2-diyl bis(triflates) as substrates for Sonogashira coupling reactions. ${ }^{6}$ Herein, we report the synthesis of the bis(triflate) ester of 4,5-diiodobenzene-1,2-diol and the application of this previously unreported electrophile in the regiocontrolled synthesis of tetraalkynylarenes (Scheme 1).

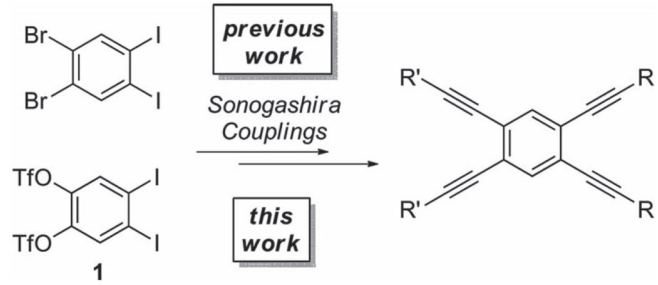

Scheme 1. Synthetic approach to tetraalkynylarenes.

\section{Results and Discussion}

Diiodo bis(triflate) $\mathbf{1}$ was readily prepared in three steps from 1,2-dimethoxybenzene as shown in Scheme 2. Electrophilic iodination of 1,2-dimethoxybenzene proceeded in $86 \%$ yield. ${ }^{7}$ Bis(demethylation), followed by disulfonylation of the resulting catechol proceeded quantitatively to furnish 1. We used this procedure to prepare up to $20 \mathrm{~g}$ of $\mathbf{1}$, which is stable in the dark at room temperature for months.

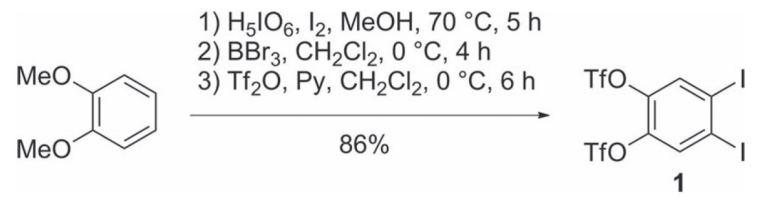

Scheme 2. Synthesis of $\mathbf{1}$.

The selectivity of Sonagashira coupling reactions of $\mathbf{1}$ was investigated by using the benzyl ether of 4-pentynol as a model alkyne (Table 1$)$. $\left[\mathrm{PdCl}_{2}\left(\mathrm{PPh}_{3}\right)_{2}\right]$ proved to be an efficient catalyst at a loading of 6 mol-\% (Entry 1$)$. Good yields 
Table 1. Optimization of the alkyne coupling reaction with 1 . $^{\text {[a] }}$

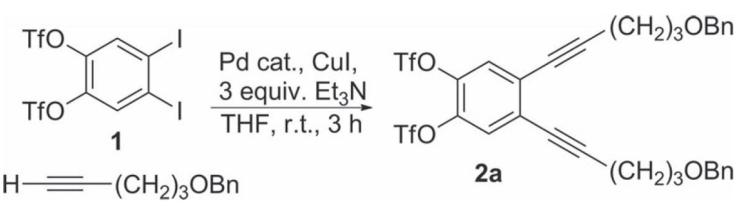

\begin{tabular}{lllll}
\hline $\begin{array}{c}\text { Entry } \\
1\end{array}$ & $\begin{array}{c}\text { Pd cat. (amount } \\
[\mathrm{mol}-\%])\end{array}$ & $\begin{array}{c}\text { Alkyne } \\
\text { equiv. }\end{array}$ & $\begin{array}{l}\text { Conv. } \\
{[\%]}\end{array}$ & $\begin{array}{l}\text { Yield }{ }^{[\mathrm{b}]} \\
{[\%]}\end{array}$ \\
\hline 1 & {$\left[\mathrm{PdCl}_{2}\left(\mathrm{PPh}_{3}\right)_{2}\right](6)$} & 2.3 & $>99$ & 84 \\
2 & {$\left[\mathrm{PdCl}_{2}\left(\mathrm{PPh}_{3}\right)_{2}\right](3)$} & 2.3 & 68 & 16 \\
3 & {$\left[\mathrm{PdCl}_{2}\left(\mathrm{PPh}_{3}\right)_{2}\right](1)$} & 2.3 & 67 & 2 \\
4 & {$\left[\mathrm{PdCl}_{2}\left(\mathrm{PPh}_{3}\right)_{2}\right](3)$} & 2.3 & $>99$ & 70 \\
$5[\mathrm{~d}]$ & {$\left[\mathrm{PdCl}_{2}\left(\mathrm{PPh}_{3}\right)_{2}\right](3)$} & 2.3 & $>99$ & nd \\
6 & {$\left[\mathrm{PdCl}_{2}\left(\mathrm{PPh}_{3}\right)_{2}\right](6)$} & 2.1 & $>95$ & 69 \\
7 & {$\left[\mathrm{PdCl}_{2}\left(\mathrm{PPh}_{3}\right)_{2}\right](6)$} & 2.5 & $>99$ & 81 \\
8 & {$\left[{\left.\mathrm{Pd}\left(\mathrm{PPh}_{3}\right)_{4}\right](6)}_{9}\right.$} & 2.3 & 52 & trace \\
9 & {$\left[\mathrm{PdCl}_{2}(\mathrm{dppf})\right](6)$} & 2.3 & 47 & trace \\
\hline
\end{tabular}

[a] Reagents: 1 (0.4 mmol), CuI/Pd (2:1); nd = not determined. [b] Isolated yield. [c] Time $=10 \mathrm{~h}$. [d] $\mathrm{T}=60^{\circ} \mathrm{C}$.

of the 1,2-bis(alkynyl) product could be obtained at lower catalyst loadings but at the cost of extended reaction times (Entry 4). Complete conversion of the starting material at lower catalyst loadings could be achieved by performing the reaction at higher temperatures (Entry 5), but these conditions resulted in the formation of copious amounts of byproducts that were difficult to separate from the desired diyne. Alternative sources of $\mathrm{Pd}^{\mathrm{II}}$ or $\mathrm{Pd}^{0}$ provided lower conversions and only trace amounts of the product (Entries 8 and 9). A 2:1 ratio of $\mathrm{Cu}^{\mathrm{I}} / \mathrm{Pd}^{\mathrm{II}}$ proved optimal for the transformation. Complete selectivity for the coupling of the aryl iodides was found when 2.3 equiv. of alkyne were used; no appreciable amounts of products resulting from coupling to the triflates were detected. However, performing the reaction in the presence of a large excess (5 equiv.) of alkyne did lead to the formation of significant amounts of inseparable byproducts, which appeared to include the triand tetraalkynylbenzene (not shown).

The selectivity of the reaction is significant. One might envisage that the introduction of an alkyne in the first Sonagashira coupling might direct the second coupling away from the ortho iodide and towards one of the electron-deficient, but sterically unencumbered triflate units; electron-poor aryl halides are typically favored as electrophiles in alkyne cross-coupling reactions. ${ }^{8}$ However, the selective Sonogashira coupling of aryl iodides in the presence of aryl triflates has previously been demonstrated. ${ }^{5}$ Interestingly, the reaction of $\mathbf{1}$ with only 1.1 equiv. of (4-methoxyphenyl) ethyne under typical reaction conditions generated the diyne and monoyne in a 1.3:1 ratio in a combined yield of $81 \%$ (not shown). The modest preference for dialkynylation could reflect a directing effect of the ortho-alkyne ${ }^{9}$ or, alternatively, a more facile insertion into the hindered aryl iodide due to the diffusion-controlled proximity of the Pd catalyst to the electrophile. ${ }^{10}$

With the optimized reaction conditions in hand, we investigated the scope of the selective cross-coupling reaction of 1 with a variety of alkynes (Table 2). Although most reactions were complete in $3 \mathrm{~h}$, some alkynes required
Table 2. Substrate scope for the selective coupling of 1 . $^{\text {[a] }}$

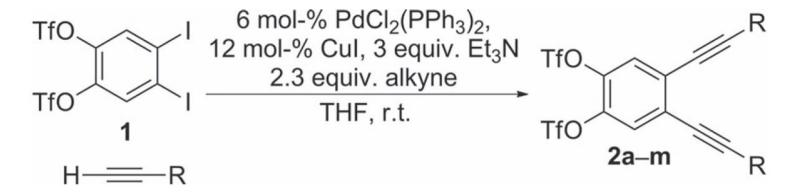

\begin{tabular}{lllc}
\hline Entry & $\mathrm{R}$ & $t[\mathrm{~h}]$ & Product, yield ${ }^{[\mathrm{b}]}[\%]$ \\
\hline 1 & $\left(\mathrm{CH}_{2}\right)_{3} \mathrm{OBn}$ & 3 & $\mathbf{2 a}, 84$ \\
2 & $\left(\mathrm{CH}_{2}\right)_{3} \mathrm{OBz}$ & 5 & $\mathbf{2 b}, 71$ \\
3 & $\left(\mathrm{CH}_{2}\right)_{3} \mathrm{CN}$ & 3 & $\mathbf{2 c}, 78$ \\
4 & $\left(\mathrm{CH}_{2}\right)_{4} \mathrm{OTBS}$ & 3 & $\mathbf{2 d}, 94$ \\
5 & $\left(\mathrm{CH}_{2}\right)_{4} \mathrm{Cl}$ & 3 & $\mathbf{2 e}, 76$ \\
6 & $\mathrm{TIPS}_{7}$ & 3 & $\mathbf{2 f}, 46$ \\
7 & $4-\mathrm{MeOC} \mathrm{H}_{4}$ & 3 & $\mathbf{2 g}, 78$ \\
8 & $2-\mathrm{Py}$ & 3 & $\mathbf{2 h}, 69$ \\
9 & $2-\mathrm{Py}$ & 3 & $\mathbf{2 h}, 84$ [c] \\
10 & $\left(\mathrm{CH}_{2}\right)_{2} \mathrm{OTHP}$ & 3 & $\mathbf{2 i}, 85$ \\
11 & $\mathrm{C}\left(\mathrm{CH}_{3}\right)_{2} \mathrm{OH}$ & 3 & $\mathbf{2 j}, 72$ \\
12 & $\left.\mathrm{CH}_{4} \mathrm{OEt}\right)_{2}$ & 3 & $\mathbf{2 k}, 69$ \\
13 & $\mathrm{CH}_{2} \mathrm{OBz}$ & 3 & $\mathbf{2 1}, 46$ \\
14 & $\mathrm{CH}_{2} \mathrm{OBn}$ & 5 & $\mathbf{2 m}, 43$ \\
\hline
\end{tabular}

[a] Reagents: 1 (0.4 mmol), THF (1 mL). [b] Isolated yield. [c] On a $3.2 \mathrm{mmol}$ scale.

longer times for the complete consumption of the starting material (Entries 2 and 14). The reactions proceeded smoothly with alkynes functionalized with esters (Entry 2), nitriles (Entry 3), silyl ethers (Entry 4), alkyl chlorides (Entry 5), THP ethers (Entry 10), and an acetal (Entry 12). Aromatic alkynes also proved to be excellent coupling partners (Entries 7-9). Coupling reactions with nonpolar alkynes (e.g., heptyne) appeared to proceed to completion (TLC); however, because of the difficulty in completely separating the products from recovered alkyne and nonpolar byproducts by silica chromatography, these examples are not reported. Cross-coupling with a tertiary propargyl alcohol proceeded in good yield (Entry 11), whereas reactions with unhindered propargyl ethers (Entries 13 and 14) provided lower yields of the bis(alkynes). Given the frequent application of propargyl alcohols and ethers as nucleophiles in Sonagashira coupling reactions, 1 we are uncertain as to why their use has limitations in this setting.

We next investigated the conditions for displacing both triflates in a second set of Sonogashira coupling reactions (Table 3). By using conditions related to those reported by Powell and Rychnovsky, ${ }^{6}$ a diyne $2 \mathbf{a}$ was coupled with the TBS ether of 5-hexynol to yield tetrayne $3 \mathbf{a}$ in $72 \%$ yield (Entry 1). Further modification of the reported conditions did not lead to any notable improvement (data not shown). The scope of the second set of coupling reactions was then investigated (Entries 2-9).

Finally, we compared our new approach directly against an existing methodology for the synthesis of $\mathbf{3 i}$, a target previously prepared in four steps and $11 \%$ yield from 1,2-dibromo-4,5-diiodobenzene (Scheme 3). ${ }^{2 a}$ By using our method, $3 \mathbf{i}$ could be prepared from diiododitriflate 1 in two steps and 61\% yield (or five steps and 53\% overall yield from 1,2-dimethoxybenzene). 
Table 3. Synthesis of tetraalkynylarenes by dual coupling of dialkynylarenediyl bis(triflates). ${ }^{\text {[a] }}$
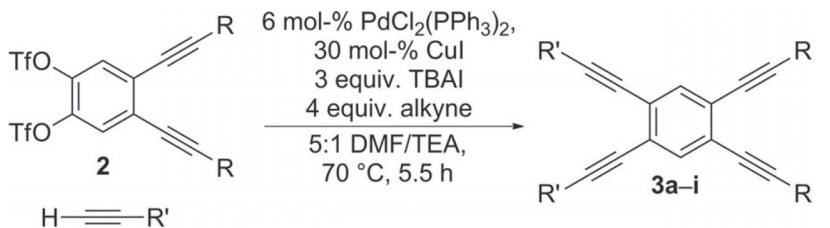

\begin{tabular}{llll}
\hline Entry & $\mathrm{R}$ & $\mathrm{R}^{\prime}$ & Product, yield \\
\hline 1 & $\left(\mathrm{CH}_{2}\right)_{3} \mathrm{OBn}$ & $\left(\mathrm{CH}_{2}\right)_{4} \mathrm{OTBS}$ & $\mathbf{3 a}, 72$ \\
2 & $\left(\mathrm{CH}_{2}\right)_{3} \mathrm{OBn}$ & $\left(\mathrm{CH}_{2}\right)_{3} \mathrm{OBz}$ & $\mathbf{3 b}, 64$ \\
3 & $\left(\mathrm{CH}_{2}\right)_{3} \mathrm{OBn}$ & $\mathrm{TMS}$ & $\mathbf{3 c}, 64$ \\
4 & $\left(\mathrm{CH}_{2}\right)_{3} \mathrm{OBn}$ & $\mathrm{Ph}$ & $\mathbf{3 d}, 83$ \\
5 & $\left(\mathrm{CH}_{2}\right)_{3} \mathrm{OBn}$ & $4-\mathrm{MeOC}_{6} \mathrm{H}_{4}$ & $\mathbf{3 e}, 79$ \\
6 & $\left(\mathrm{CH}_{2}\right)_{3} \mathrm{OBn}$ & $4-\mathrm{FC}_{6} \mathrm{H}_{4}$ & $\mathbf{3 f}, 74$ \\
$7[\mathrm{c}]$ & $\left(\mathrm{CH}_{2}\right)_{4} \mathrm{OTBS}_{4}$ & $\left(\mathrm{CH}_{2}\right)_{3} \mathrm{OBz}$ & $\mathbf{3 g}, 68$ \\
8 & $4-\mathrm{MeOC}_{6} \mathrm{H}_{4}$ & $\mathrm{TMS}$ & $\mathbf{3 h}, 57$ \\
9 & $2-\mathrm{Py}$ & $4-\left(\mathrm{Bu}_{2} \mathrm{~N}\right) \mathrm{C}_{6} \mathrm{H}_{4}$ & $\mathbf{3 i}, 73$ \\
\hline
\end{tabular}

[a] Reagents and conditions: on a $0.25 \mathrm{mmol}$ scale, DMF/TEA (5:1) $(1.5 \mathrm{~mL})$, sealed vial. [b] Isolated yield. [c] KI (3 equiv.) used instead of TBAI.

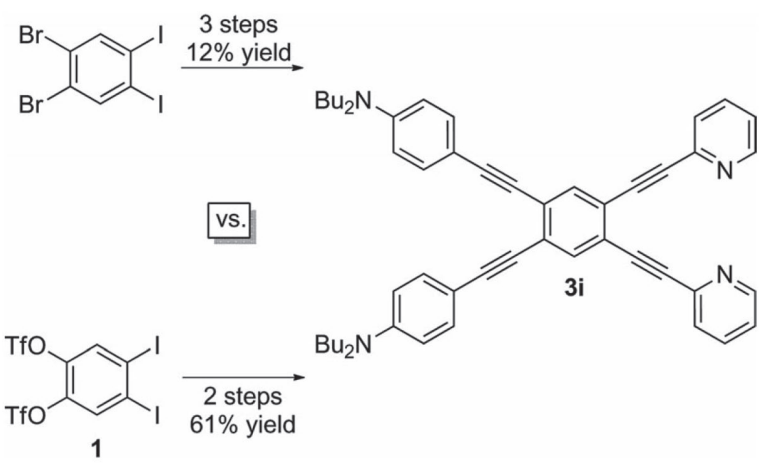

Scheme 3. Comparison of the protocol developed here with an existing methodology.

\section{Conclusions}

The selective Sonogashira cross-coupling reaction of 1,2-diiodobenzene-4,5-diyl bis(triflate) proceeds with almost complete selectivity displacing both iodine atoms to furnish a 4,5-bis(alkynyl)benzene-1,2-diyl bis(triflate), which can be subjected to a second set of alkyne coupling reactions to efficiently give nonsymmetric 1,2,4,5-tetraalkynylarenes. Structurally related 1,2-diyl bis(triflates) have been shown to undergo dual Suzuki coupling reactions, ${ }^{6 \mathrm{~b}}$ which suggests our results could potentially be extended to other transition-metal-catalyzed cross-coupling reactions.

\section{Experimental Section}

General: All the reactions were carried out in flame-dried glassware under dry nitrogen with magnetic stirring. Solvents were used as purchased with the exception of THF and $\mathrm{CH}_{2} \mathrm{Cl}_{2}$, which were distilled from $\mathrm{Na} / \mathrm{Ph}_{2} \mathrm{CO}$ and $\mathrm{CaH}_{2}$, respectively. TLC was performed on $0.25 \mathrm{~mm}$ hard-layer silica G plates; developed plates were visualized with a UV lamp and/or by staining: vanillin (general stain, after charring), $1 \%$ aq. $\mathrm{KMnO}_{4}$ (for unsaturated compounds), $\mathrm{I}_{2}$, or phosphomolybdic acid (general stain, after charring). NMR spectra were recorded in $\mathrm{CDCl}_{3}$ (by using residual $\mathrm{CHCl}_{3}: \delta=7.286 \mathrm{ppm}$ ) at 300/400/500/600 $\mathrm{MHz}\left({ }^{1} \mathrm{H}\right)$ or $75 / 100 / 125 / 150 \mathrm{MHz}\left({ }^{13} \mathrm{C}\right)$, as indicated. ${ }^{1} \mathrm{H}$ NMR chemical shifts are reported as $\delta$ in ppm as follows: chemical shift [multiplicity, coupling constant(s) in $\mathrm{Hz}$, integration]. ${ }^{13} \mathrm{C}$ NMR chemical shifts are reported as $\delta$ in ppm. IR spectra were recorded as neat ATR films with selected absorbances reported in wavenumbers $\left(\mathrm{cm}^{-1}\right)$.

1,2-Diiodo-4,5-dimethoxybenzene: This compound was prepared according to the procedure of Lacour et al. $7 \mathrm{H}_{5} \mathrm{IO}_{6}(0.41$ equiv., $25.6 \mathrm{mmol}, 5.84 \mathrm{~g})$ and $\mathrm{MeOH}(36 \mathrm{~mL})$ were added to a flame-dried $100 \mathrm{~mL}$ round-bottomed flask equipped with a short air condenser. The mixture was stirred at room temp., and then $\mathrm{I}_{2}$ ( 0.8 equiv., $50.2 \mathrm{mmol}, 12.76 \mathrm{~g}$ ) was added. The reaction mixture was stirred vigorously for $10 \mathrm{~min}$, after which 1,2-dimethoxybenzene ( 1 equiv., $63 \mathrm{mmol}, 8.7 \mathrm{~g}, 8.0 \mathrm{~mL}$ ) was added in one portion through a syringe. The reaction mixture was heated at $70^{\circ} \mathrm{C}$ in an oil bath for $5 \mathrm{~h}$, which resulted in the formation of a white solid that made stirring difficult; however, the reaction proceeded even without efficient stirring. The hot solution was poured into dilute aqueous $\mathrm{Na}_{2} \mathrm{~S}_{2} \mathrm{O}_{5}$ (ca. $100 \mathrm{~mL}$ ), and the mixture was cooled to room temp. The solid collected by filtration through a glass frit was washed quickly with two $30 \mathrm{~mL}$ portions of cold $\mathrm{MeOH}$ and dried in vacuo to afford 1,2-diiodo-4,5dimethoxybenzene $(21.07 \mathrm{~g}, 54 \mathrm{mmol}, 86 \%)$ as a white solid that was deemed pure by NMR spectroscopy and used without further purification. $R_{\mathrm{f}}=0.49$ (20\% ethyl acetate/hexane). M.p. 134.5-136.0 ${ }^{\circ} \mathrm{C}$ (ref.7 $\left.134^{\circ} \mathrm{C}\right) .{ }^{1} \mathrm{H}$ NMR (600 MHz): $\delta=7.25$ (s, 2 $\mathrm{H}), 3.85$ (s, $6 \mathrm{H}) \mathrm{ppm} .{ }^{13} \mathrm{C}$ NMR $(150 \mathrm{MHz}): \delta=149.6,121.7,96.1$, $56.2 \mathrm{ppm}$.

1,2-Dihydroxy-4,5-diiodobenzene: A flame-dried $250 \mathrm{~mL}$ round-bottomed flask was charged with 1,2-diiodo-4,5-dimethoxybenzene (1 equiv., $10 \mathrm{mmol}, 3.90 \mathrm{~g}$ ) and then evacuated/ back-filled with nitrogen $(3 \times)$ before addition of $\mathrm{CH}_{2} \mathrm{Cl}_{2}(70 \mathrm{~mL})$. The solution was cooled to $0{ }^{\circ} \mathrm{C}$, and $\mathrm{BBr}_{3}$ (2.5 equiv., $25 \mathrm{mmol}$, $25 \mathrm{~mL}$ of a $1.0 \mathrm{M}$ solution in $\mathrm{CH}_{2} \mathrm{Cl}_{2}$ ) was added through a syringe pump over $20 \mathrm{~min}$. The reaction mixture was stirred at 0 ${ }^{\circ} \mathrm{C}$ for $4 \mathrm{~h}$ and then quenched with $\mathrm{H}_{2} \mathrm{O}(50 \mathrm{~mL})$. The separated aqueous layer was extracted with $\mathrm{Et}_{2} \mathrm{O}(2 \times 75 \mathrm{~mL})$. The combined organic layers were dried with $\mathrm{MgSO}_{4}$, filtered through a pad of silica, and concentrated in vacuo to afford 1,2-dihydroxy-4,5-diiodobenzene (3.61 g, $9.99 \mathrm{mmol}$, quant.) as an offwhite solid that was deemed pure by NMR spectroscopy and used without further purification. $R_{\mathrm{f}}=0.50$ (50\% ethyl acetate/ hexane). M.p. $116.0-116.5^{\circ} \mathrm{C} .{ }^{1} \mathrm{H}$ NMR (400 MHz, [D $]$ acetone): $\delta$ $=8.48$ (br. s, $2 \mathrm{H}), 7.38(\mathrm{~s}, 2 \mathrm{H}) \mathrm{ppm} .{ }^{13} \mathrm{C} \mathrm{NMR}(150 \mathrm{MHz}$, [D $]$ acetone): $\delta=146.5,125.6,93.7 \mathrm{ppm}$.

4,5-Diiodo-1,2-phenylene Bis(trifluoromethanesulfonate) (1): 1,2-Dihydroxy-4,5-diiodobenzene (1 equiv., $7.85 \mathrm{mmol}, 2.84 \mathrm{~g}$ ), $\mathrm{CH}_{2} \mathrm{Cl}_{2}(55 \mathrm{~mL})$, and pyridine (5 equiv., $39 \mathrm{mmol}, 3.10 \mathrm{~g}, 3.16$ $\mathrm{mL}$ ) were added to a flame-dried $100 \mathrm{~mL}$ round-bottomed flask. The solution was cooled to $0{ }^{\circ} \mathrm{C}$, and $\mathrm{Tf}_{2} \mathrm{O}(2.2$ equiv., $17.3 \mathrm{mmol}$, $4.88 \mathrm{~g}, 2.91 \mathrm{~mL}$ ) was added dropwise through a syringe over 10 min. The reaction mixture was stirred for $6 \mathrm{~h}$, while warming to ambient temperature, then cooled to $0{ }^{\circ} \mathrm{C}$, and quenched with $\mathrm{H}_{2} \mathrm{O}(30 \mathrm{~mL})$. The separated aqueous layer was extracted with $\mathrm{CH}_{2} \mathrm{Cl}_{2}(2 \times 30 \mathrm{~mL})$. The combined organic layers were dried with $\mathrm{MgSO}_{4}$ and filtered through a tall pad of silica. The pad was washed carefully with $\mathrm{CH}_{2} \mathrm{Cl}_{2}$ to avoid the elution of impurities, and the filtrate was concentrated in vacuo to afford $\mathbf{1}$ (4.90 $\mathrm{g}, 7.82 \mathrm{mmol}$, quant.) as an off-white solid that was deemed pure by NMR spectroscopy and used without further purification. (Note: For reactions in which small amounts of impurities were observed after filtration, the product could be obtained in 
pure form by column chromatography utilizing $10 \%$ ethyl acetate/hexane as the mobile phase.) $R_{\mathrm{f}}=0.60(10 \%$ ethyl acetate/ hexane). M.p. $46.5-47.7^{\circ} \mathrm{C} .{ }^{1} \mathrm{H}$ NMR $(400 \mathrm{MHz}): \delta=7.91(\mathrm{~s}, 2 \mathrm{H})$ ppm. ${ }^{13} \mathrm{C}$ NMR $(100 \mathrm{MHz}): \delta=139.6,133.4,118.5\left(\mathrm{q}, J_{\mathrm{C}, \mathrm{F}}=321.0\right.$ Hz), 108.0 ppm. FTIR: $\bar{v}=1429,1335,1215,1125,1105,868,788$, $745,689 \mathrm{~cm}^{-1}$. HRMS (ESI): calcd. for $\mathrm{C}_{8} \mathrm{H}_{2} \mathrm{~F}_{6} \mathrm{I}_{2} \mathrm{NaO}_{6} \mathrm{~S}_{2}[\mathrm{M}+\mathrm{Na}]^{+}$ 648.7184; found 648.7164 .

General Procedure A. Sonogashira Coupling of 1: A flamedried $8 \mathrm{~mL}$ vial fitted with a screw-top septum cap was charged with $\left[\mathrm{Pd}\left(\mathrm{PPh}_{3}\right)_{2} \mathrm{Cl}_{2}\right]$ (0.06 equiv., $\left.0.024 \mathrm{mmol}, 16.8 \mathrm{mg}\right)$, $\mathrm{CuI}(0.12$ equiv., $0.048 \mathrm{mmol}, 9.2 \mathrm{mg}$ ), and 1 (1 equiv., $0.40 \mathrm{mmol}, 250 \mathrm{mg})$ and then evacuated/back-filled with nitrogen $(3 \times)$. A solution of the alkyne (2.3 equiv., $0.92 \mathrm{mmol})$ in THF $(1 \mathrm{~mL})$ was then added, followed by $\mathrm{Et}_{3} \mathrm{~N}$ (3 equiv., $1.2 \mathrm{mmol}, 0.17 \mathrm{~mL}$ ). The mixture was stirred at room temp. for the required time (3-5 h) until the reaction was complete (TLC) and then filtered through a plug of silica, which was washed with $\mathrm{Et}_{2} \mathrm{O}$. The combined eluents were concentrated in vacuo and purified by flash chromatography (ethyl acetate/hexane) to afford the diyne.

$4,5$ - B is ( 5 - be n z l oxy pen t- 1 - y n y 1$)-1,2$ - phen ylene Bis(trifluoromethanesulfonate) (2a): Yield: $241 \mathrm{mg}, 0.336 \mathrm{mmol}$, $84 \%$. Mobile phase: step gradient hexane to $10 \%$ ethyl acetate/ hexane. $R_{\mathrm{f}}=0.27\left(10 \%\right.$ ethyl acetate/hexane). ${ }^{1} \mathrm{H}$ NMR $(600$ $\mathrm{MHz}): \delta=7.42(\mathrm{~s}, 2 \mathrm{H}), 7.27-7.39(10 \mathrm{H}), 4.56(\mathrm{~s}, 4 \mathrm{H}), 3.65(\mathrm{t}, J$ $=6.0 \mathrm{~Hz}, 4 \mathrm{H}), 2.62(\mathrm{t}, J=7.1 \mathrm{~Hz}, 4 \mathrm{H}), 1.92-1.98(\mathrm{~m}, 4 \mathrm{H}) \mathrm{ppm}$. ${ }^{13} \mathrm{C}$ NMR $(150 \mathrm{MHz}): \delta=138.8,138.3,128.4,128.2,127.59,127.56$, $126.3,118.5\left(\mathrm{q}, J_{\mathrm{C} . \mathrm{F}}=321.1 \mathrm{~Hz}\right), 98.1,77.4,73.0,68.5,28.6,16.5$ ppm. FTIR: $v=2859,2230,1489,1433,1210,1178,1135,1080$, $732 \mathrm{~cm}^{-1}$. HRMS (ESI): calcd. for $\mathrm{C}_{32} \mathrm{H}_{28} \mathrm{~F}_{6} \mathrm{O}_{8} \mathrm{~S}_{2} \mathrm{Na}[\mathrm{M}+\mathrm{Na}]^{+}$ 741.1027; found 741.1039.

5,5'-[4,5-Bis(trifluoromethylsulfonyloxy)-1,2-phenylene]dipent-4-ynyl Dibenzoate (2b): Yield: $211 \mathrm{mg}, 0.284 \mathrm{mmol}, 71 \%$. Mobile phase: step gradient hexane to $12 \%$ ethyl acetate/hexane. $R_{\mathrm{f}}=0.20$ (10\% ethyl acetate/hexane). ${ }^{1} \mathrm{H}$ NMR $(400 \mathrm{MHz})$ : $\delta=8.01-8.11(4 \mathrm{H}), 7.53-7.60(2 \mathrm{H}), 7.39-7.48(6 \mathrm{H}), 4.51(\mathrm{t}, J=$ $6.3 \mathrm{~Hz}, 4 \mathrm{H}$ ), $2.71(\mathrm{t}, J=7.0 \mathrm{~Hz}, 4 \mathrm{H}), 2.12$ (quint, $J=6.5 \mathrm{~Hz}, 4 \mathrm{H}$ ) ppm. ${ }^{13} \mathrm{C}$ NMR $(150 \mathrm{MHz}): \delta=166.5,138.9,133.0,130.1,129.6$, $128.4,128.0,126.5,118.5\left(\mathrm{q}, J_{\mathrm{C}, \mathrm{F}}=321.4 \mathrm{~Hz}\right), 97.2,77.8,63.5,27.6$, 16.7 ppm. FTIR: $\bar{v}=2210,1716,1489,1433,1271,1246,1209,1177$, 1134, 1085, 1070, 1027, 863, 808, $708 \mathrm{~cm}^{-1}$. HRMS (ESI): calcd. for $\mathrm{C}_{32} \mathrm{H}_{24} \mathrm{~F}_{6} \mathrm{O}_{10} \mathrm{~S}_{2} \mathrm{Na}[\mathrm{M}+\mathrm{Na}]^{+}$769.0613; found 769.0609.

4,5 - B i s ( 5 - c y a n o p e n t - 1 - y n y 1) - 1, 2-phe n y le ne Bis(trifluoromethanesulfonate) (2c): Yield: $173 \mathrm{mg}, 0.312 \mathrm{mmol}$, $78 \%$. M.p. $56.8-58.0{ }^{\circ} \mathrm{C}$. Mobile phase: step gradient $25-35 \%$ ethyl acetate/hexane. $R_{\mathrm{f}}=0.35\left(40 \%\right.$ ethyl acetate/hexane). ${ }^{1} \mathrm{H}$ NMR (600 MHz): $\delta=7.49(\mathrm{~s}, 2 \mathrm{H}), 2.73(\mathrm{t}, J=6.7 \mathrm{~Hz}, 4 \mathrm{H}), 2.62$ $\left(\mathrm{t}, J=6.7 \mathrm{~Hz}, 4 \mathrm{H}\right.$ ), 2.03 (quint, $J=6.7 \mathrm{~Hz}, 4 \mathrm{H}$ ) ppm. ${ }^{13} \mathrm{C} \mathrm{NMR}$ $(150 \mathrm{MHz}): \delta=139.2,127.5,126.7,121.7,118.5\left(\mathrm{q}, J_{\mathrm{C}, \mathrm{F}}=321.0\right.$ $\mathrm{Hz}), 95.4,78.7,24.2,18.6,16.2$ ppm. FTIR: $\bar{v}=2231,1490,1431$, 1401, 1246, 1223, 1212, 1131, 1084, 907, 858, 799, $749 \mathrm{~cm}^{-1}$. HRMS (ESI): calcd. for $\mathrm{C}_{20} \mathrm{H}_{14} \mathrm{~F}_{6} \mathrm{~N}_{2} \mathrm{O}_{6} \mathrm{~S}_{2} \mathrm{Na}[\mathrm{M}+\mathrm{Na}]^{+}$579.0095; found 579.0092 .

4,5-Bis[6-(tert-butyldimethylsilyloxy)hex-1-ynyl]-1,2-phenyl eneBis(trifluoromethanesulfonate) (2d): Yield: $298 \mathrm{mg}, 0.376$ mmol, $94 \%$. Mobile phase: step gradient hexane to $2 \%$ ethyl acetate/hexane. $R_{\mathrm{f}}=0.27$ (2.5\% ethyl acetate/hexane). ${ }^{1} \mathrm{H}$ NMR (400 $\mathrm{MHz}): \delta=7.44$ (s, $2 \mathrm{H}), 3.66-3.72(4 \mathrm{H}), 2.49-2.61(4 \mathrm{H}), 1.66-1.77$ $(8 \mathrm{H}), 0.92$ (s, $18 \mathrm{H}), 0.08(\mathrm{~s}, 12 \mathrm{H}) \mathrm{ppm} .{ }^{13} \mathrm{C}$ NMR $(150 \mathrm{MHz})$ : $\delta=138.8,128.3,126.3,118.5\left(\mathrm{q}, J_{\mathrm{C}, \mathrm{F}}=320.9 \mathrm{~Hz}\right), 98.6,77.3,62.5$, $31.9,25.9,24.9,19.4,18.3,-5.3$ ppm. FTIR: $\bar{v}=2945,2930,2858$, 2230, 1489, 1436, 11247, 1211, 1179, 1137, 1087, 834, 807, $774 \mathrm{~cm}^{-}$ 1. HRMS (ESI): calcd. for $\mathrm{C}_{32} \mathrm{H}_{48} \mathrm{~F}_{6} \mathrm{O}_{8} \mathrm{~S}_{2} \mathrm{Na}[\mathrm{M}+\mathrm{Na}]^{+}$817.2131; found 817.2120.
4,5 - B i s ( 6 - c h l o r o h e x - 1-y n y l) - 1, 2-p h e n y len e Bis(trifluoromethanesulfonate) (2e): Yield: $185 \mathrm{mg}, 0.304 \mathrm{mmol}$, $76 \%$. Mobile phase: $4 \%$ ethyl acetate/hexane, $R_{\mathrm{f}}=0.27$ (5\% ethyl acetate/hexane). ${ }^{1} \mathrm{H}$ NMR (600 MHz): $\delta=7.46(\mathrm{~s}, 2 \mathrm{H}), 3.63(\mathrm{t}, J$ $=6.6 \mathrm{~Hz}, 4 \mathrm{H}), 2.57(\mathrm{t}, J=7.0 \mathrm{~Hz}, 4 \mathrm{H}), 1.97-2.04(4 \mathrm{H}), 1.80-1.86$ (4 H) ppm. ${ }^{13} \mathrm{C}$ NMR $(150 \mathrm{MHz}): \delta=138.9,128.0,126.5,118.5$ $\left(\mathrm{q}, J_{\mathrm{C}, \mathrm{F}}=320.7 \mathrm{~Hz}\right), 97.7,77.7,44.4,31.6,25.6,19.0 \mathrm{ppm}$. FTIR: $v=2946,2230,1489,1432,1302,1246,1209,1179,1085,862,802$, $597 \mathrm{~cm}^{-1}$. HRMS (ESI): calcd. for $\mathrm{C}_{20} \mathrm{H}_{18} \mathrm{~F}_{6} \mathrm{O}_{8} \mathrm{~S}_{2} \mathrm{Cl}_{2} \mathrm{Na}[\mathrm{M}+\mathrm{Na}]^{+}$ 624.9724; found 624.9716 .

4,5-Bis[2-(triisopropylsilyl)ethyny1]-1,2-phenylene Bis(trifluoromethanesulfonate) (2f): Yield: $136 \mathrm{mg}, 0.184 \mathrm{mmol}$, $46 \%$. M.p. $42.3-43.5^{\circ} \mathrm{C}$. Mobile phase: $100 \%$ hexane. $R_{\mathrm{f}}=0.43$ (100\% hexane). ${ }^{1} \mathrm{H}$ NMR (600 MHz): $\delta=7.52$ (s, $\left.2 \mathrm{H}\right), 1.14-1.21$ $(42 \mathrm{H})$ ppm. ${ }^{13} \mathrm{C}$ NMR $(150 \mathrm{MHz}): \delta=139.3,128.0,127.3,118.5$ $\left(\mathrm{q}, J_{\mathrm{C}, \mathrm{F}}=320.9 \mathrm{~Hz}\right), 102.1,100.7,18.7,11.2 \mathrm{ppm}$. FTIR: $\bar{v}=2944$ $2867,2363,1485,1436,1385,1211,1167,1135,881,834,574 \mathrm{~cm}^{-1}$. HRMS (EI): calcd. for $\mathrm{C}_{30} \mathrm{H}_{44} \mathrm{~F}_{6} \mathrm{O}_{6} \mathrm{~S}_{2} \mathrm{Si}_{2} \mathrm{Na}[\mathrm{M}]^{+}$734.2022; found 734.2020.

4,5-Bis [2-(4-methoxyphenyl)ethy nyl]-1,2-phenylene Bis(trifluoromethanesulfonate) (2g): Yield: $197 \mathrm{mg}, 0.312 \mathrm{mmol}$, $78 \%$. M.p. $113.4-114.3^{\circ} \mathrm{C}$. Mobile phase: step gradient hexane to $7 \%$ ethyl acetate/hexane. $R_{\mathrm{f}}=0.30$ (10\% ethyl acetate/hexane). ${ }^{1} \mathrm{H}$ NMR $(400 \mathrm{MHz}): \delta=7.59$ (s, $\left.2 \mathrm{H}\right), 7.51-7.57(4 \mathrm{H}), 6.90-6.97$ (4 H), 3.88 (s, $6 \mathrm{H}) \mathrm{ppm} .{ }^{13} \mathrm{C}$ NMR $(150 \mathrm{MHz}): \delta=160.6,139.0$, $133.5,127.8,125.9,118.5\left(\mathrm{q}, J_{\mathrm{C}, \mathrm{F}}=321.0 \mathrm{~Hz}\right), 114.3,114.0,97.7$, 84.6, 55.4 ppm. FTIR: $\bar{v}=2205,1605,1513,1485,1435,1414,1290$, $1248,1213,1130,1027,896,828 \mathrm{~cm}^{-1}$. HRMS (ESI): calcd. for $\mathrm{C}_{26} \mathrm{H}_{16} \mathrm{~F}_{6} \mathrm{O}_{8} \mathrm{~S}_{2} \mathrm{Na}[\mathrm{M}+\mathrm{Na}]^{+}$657.0088; found 657.0083.

4, 5-B is [2-(p y ridin-2-yl) ethyny 1]-1,2-phenylene Bis(trifluoromethanesulfonate) (2h): Yield: $158 \mathrm{mg}, 0.276 \mathrm{mmol}$, $69 \%$. M.p. $119.1-120.2{ }^{\circ} \mathrm{C}$. Mobile phase: $40 \%$ ethyl acetate/hexane. $R_{\mathrm{f}}=0.30$ (40\% ethyl acetate/hexane). ${ }^{1} \mathrm{H}$ NMR $(400 \mathrm{MHz})$ : $\delta=8.65-8.77(2 \mathrm{H}), 7.67-7.83(6 \mathrm{H}), 7.31-7.40(\mathrm{~m}, 2 \mathrm{H}) \mathrm{ppm}$. ${ }^{13} \mathrm{C}$ NMR (150 MHz): $\delta=150.4,142.2,140.0,136.3,128.1,127.3$, $126.8,123.8,118.5\left(\mathrm{q}, J_{\mathrm{C}, \mathrm{F}}=320.8 \mathrm{~Hz}\right), 96.4,84.2 \mathrm{ppm}$. FTIR: $\bar{v}=$ 1581, 1561, 1493, 1429, 1208, 1131, 1054, 1040, 904, 885, 833, 782, $732 \mathrm{~cm}^{-1}$. HRMS (ESI): calcd. for $\mathrm{C}_{22} \mathrm{H}_{10} \mathrm{~F}_{6} \mathrm{~N}_{2} \mathrm{O}_{6} \mathrm{~S}_{2} \mathrm{Na}[\mathrm{M}+\mathrm{Na}]^{+}$ 598.9782; found 598.9783.

4,5-Bis[4-(tetrahydro-2H-pyran-2-yloxy)but-1-ynyl]-1,2-phenylene Bis(trifluoromethanesulfonate) (2i): Yield: $230 \mathrm{mg}, 0.340$ mmol, $85 \%$. Mobile phase: step gradient $10-20 \%$ ethyl acetate/ hexane. $R_{\mathrm{f}}=0.22$ (15\% ethyl acetate/hexane). ${ }^{1} \mathrm{H}$ NMR $(600$ $\mathrm{MHz}): \delta=7.45(\mathrm{~s}, 2 \mathrm{H}), 4.69-4.73(2 \mathrm{H}), 3.88-3.98(4 \mathrm{H}), 3.69(\mathrm{dt}$, J $=9.7,7.0 \mathrm{~Hz}, 2 \mathrm{H}), 3.52-3.58(2 \mathrm{H}), 2.81(\mathrm{t}, J=7.0 \mathrm{~Hz}, 4 \mathrm{H}), 1.81-$ $1.91(2 \mathrm{H}), 1.71-1.79(2 \mathrm{H}), 1.51-1.69(8 \mathrm{H}) \mathrm{ppm} .{ }^{13} \mathrm{C}$ NMR $(150$ $\mathrm{MHz}): \delta=138.9,128.0,126.4,118.5\left(\mathrm{q}, J_{\mathrm{C}, \mathrm{F}}=320.4 \mathrm{~Hz}\right), 98.9,95.6$, 77.8, 65.3, 62.2, 30.5, 25.4, 21.2, 19.4 ppm. FTIR: $\bar{v}=2944,2873$, 2234, 1489, 1434, 1247, 1210, 1180, 1133, 1082, 976, 892, 859, 804, $733 \mathrm{~cm}^{-1}$. HRMS (ESI): calcd. for $\mathrm{C}_{22} \mathrm{H}_{28} \mathrm{~F}_{6} \mathrm{O}_{10} \mathrm{~S}_{2} \mathrm{Na}[\mathrm{M}+\mathrm{Na}]^{+}$ 701.0926; found 701.0925.

4,5-Bis(3-hydroxy-3-methylbut-1-ynyl)-1,2-phenylene Bis(trifluoromethanesulfonate) (2j): Yield: $155 \mathrm{mg}, 0.288 \mathrm{mmol}$, $72 \%$. Mobile phase: step gradient $30-40 \%$ ethyl acetate/hexane. $R_{\mathrm{f}}=0.22$ (30\% ethyl acetate/hexane). ${ }^{1} \mathrm{H}$ NMR $(600 \mathrm{MHz})$ : $\delta=7.48$ (s, $2 \mathrm{H}), 3.65$ (br. s, $2 \mathrm{H}), 1.66$ (s, $12 \mathrm{H}) \mathrm{ppm} .{ }^{13} \mathrm{C} \mathrm{NMR}$ $(150 \mathrm{MHz}): \delta=139.3,127.5,125.8,118.5\left(\mathrm{q}, J_{\mathrm{CF}}=321.0 \mathrm{~Hz}\right)$, 102.2, 78.2, 65.6, 31.1 ppm. FTIR: $\bar{v}=3371,2985,1486,1431,1210$, $1134,1062,952,866,826,799,734 \mathrm{~cm}^{-1}$. HRMS (ESI): calcd. for $\mathrm{C}_{18} \mathrm{H}_{16} \mathrm{~F}_{6} \mathrm{O}_{8} \mathrm{~S}_{2} \mathrm{Na}[\mathrm{M}+\mathrm{Na}]^{+}$561.0088; found 561.0094.

4,5-B is (3,3-diethoxy prop-1-y ny l)-1,2-phenylene Bis(trifluoromethanesulfonate) (2k): Yield: $172 \mathrm{mg}, 0.276$ $\mathrm{mmol}, 69 \%$. Mobile phase: step gradient hexane to $5 \%$ ethyl ac- 
etate/hexane. $R_{\mathrm{f}}=0.22$ (5\% ethyl acetate/hexane). ${ }^{1} \mathrm{H}$ NMR (600 MHz): $\delta=7.58(\mathrm{~s}, 2 \mathrm{H}), 5.52(\mathrm{~s}, 2 \mathrm{H}), 3.79-3.9(4 \mathrm{H}), 3.64-$ $3.74(4 \mathrm{H}), 1.29(\mathrm{t}, J=7.2 \mathrm{~Hz}, 12 \mathrm{H}) \mathrm{ppm} .{ }^{13} \mathrm{C} \mathrm{NMR}(150 \mathrm{MHz})$ : $\delta=139.8,127.0,126.7,118.5\left(\mathrm{q}, J_{\mathrm{C}, \mathrm{F}}=320.9 \mathrm{~Hz}\right), 92.51,91.5,80.3$, 61.3, 15.1 ppm. FTIR: $\bar{v}=3675,2979,2901,1490,1435,1248,1211$, $1178,1135,1076,1050,868,814 \mathrm{~cm}^{-1}$. HRMS (ESI): calcd. for $\mathrm{C}_{22} \mathrm{H}_{24} \mathrm{~F}_{6} \mathrm{O}_{10} \mathrm{~S}{ }_{2} \mathrm{Na}[\mathrm{M}+\mathrm{Na}]^{+}$649.0613; found 649.0610.

3,3'-[4,5-Bis(trifluoromethylsulfonyloxy)-1,2-phenylene]diprop-2-ynyl Dibenzoate (2l): Yield: $127 \mathrm{mg}, 0.184 \mathrm{mmol}, 46 \%$. M.p. $78.4-79.5{ }^{\circ} \mathrm{C}$. Mobile phase: step gradient hexane to $8 \%$ ethylacetate/hexane. $R_{\mathrm{f}}=0.35$ (10\% ethyl acetate/hexane). ${ }^{1} \mathrm{H}$ NMR (400 MHz): $\delta=8.04-8.17(4 \mathrm{H}), 7.54-7.64(4 \mathrm{H}), 7.42-7.53$ $(4 \mathrm{H}), 5.18$ (s, $4 \mathrm{H}) \mathrm{ppm} .{ }^{13} \mathrm{C}$ NMR $(150 \mathrm{MHz}): \delta=165.7,139.8$, $133.5,129.8,129.3,128.5,127.1,126.8,118.5\left(\mathrm{q}, J_{\mathrm{C} . \mathrm{F}}=320.8 \mathrm{~Hz}\right)$, 91.6, 81.7, 52.7 ppm. FTIR: $\bar{v}=1724,1490,1434,1265,1246,1210$, $1177,1134,1089,1069,869,803,709 \mathrm{~cm}^{-1}$. HRMS (ESI): calcd. for $\mathrm{C}_{28} \mathrm{H}_{16} \mathrm{~F}_{6} \mathrm{O}_{10} \mathrm{~S}{ }_{2} \mathrm{Na}[\mathrm{M}+\mathrm{Na}]^{+} 712.9987$; found 712.9988 .

4,5-B is ( 3-ben zy loxy prop-1-y n y 1)-1,2-phen ylene Bis(trifluoromethanesulfonate) (2m): Yield: $114 \mathrm{mg}, 0.172$ mmol, $43 \%$. Mobile phase: step gradient hexane to $10 \%$ ethyl acetate/hexane. $R_{\mathrm{f}}=0.27(10 \%$ ethyl acetate/hexane $) .{ }^{1} \mathrm{H}$ NMR (600 MHz): $\delta=7.55(\mathrm{~s}, 2 \mathrm{H}), 7.30-7.43(10 \mathrm{H}), 4.67$ (s, $4 \mathrm{H})$, 4.43 (s, $4 \mathrm{H}) \mathrm{ppm} .{ }^{13} \mathrm{C}$ NMR (150 MHz): $\delta=139.6,137.1,128.5$, $128.11,128.07,127.2,126.8,118.5\left(q, J_{\mathrm{CF}}=320.4 \mathrm{~Hz}\right), 93.5,82.2$, 72.0, 57.6 ppm. FTIR: $\bar{v}=3676,2988,2901,1489,1433,1247,1209$, $1178,1133,1072,864,807,737 \mathrm{~cm}^{-1}$. HRMS (ESI): calcd. for $\mathrm{C}_{28} \mathrm{H}_{20} \mathrm{~F}_{6} \mathrm{O}_{8} \mathrm{~S}_{2} \mathrm{Na}[\mathrm{M}+\mathrm{Na}]^{+}$685.0402; found 685.0397.

General Procedure B. Sonogashira Coupling of Bis(triflates): A flame-dried $8 \mathrm{~mL}$ vial fitted with a septum-containing screw-top cap was charged with $\left[\mathrm{Pd}\left(\mathrm{PPh}_{3}\right)_{2} \mathrm{Cl}_{2}\right]$ (0.06 equiv., $0.015 \mathrm{mmol}$, $10.6 \mathrm{mg}), \mathrm{CuI}$ (0.3 equiv., $0.075 \mathrm{mmol}, 14.3 \mathrm{mg})$, and $\mathrm{Bu}_{4} \mathrm{NI}(3$ equiv., $0.75 \mathrm{mmol}, 277 \mathrm{mg})$. The vessel was evacuated/backfilled with nitrogen $(3 \times)$, after which was added a solution of thebis(triflate) (1 equiv., $0.25 \mathrm{mmol}$ ) in DMF/TEA $(5: 1,1 \mathrm{~mL})$. The mixture was stirred at room temp. for $5 \mathrm{~min}$, whereupon a solution of alkyne (4 equiv., $1 \mathrm{mmol}$ ) in DMF/TEA (5:1, $0.5 \mathrm{~mL})$ was added. The capped vial was placed in an oil bath $\left(70^{\circ} \mathrm{C}\right)$ for $5.5 \mathrm{~h}$ and then cooled to room temp. The reaction mixture was filtered through a plug of silica, which was washed with $\mathrm{Et}_{2} \mathrm{O}$. The combined eluents were concentrated in vacuo and purified by flash chromatography.

1,2-Bis(5-benzyloxypent-1-ynyl)-4,5-bis(6-tert-butyldimethylsilyloxyhex-1-ynyl)benzene (3a): Yield: $151 \mathrm{mg}, 0.18 \mathrm{mmol}, 72 \%$. Mobile phase: $6 \%$ ethyl acetate/hexane. $R_{\mathrm{f}}=0.54$ (10\% ethyl acetate/hexane). ${ }^{1} \mathrm{H}$ NMR $(600 \mathrm{MHz}): \delta=7.32-7.40(10 \mathrm{H}), 7.27-$ $7.31(2 \mathrm{H}), 4.55(\mathrm{~s}, 4 \mathrm{H}), 3.69(\mathrm{t}, J=5.9 \mathrm{~Hz}, 4 \mathrm{H}), 3.65(\mathrm{t}, J=6.2 \mathrm{~Hz}$, $4 \mathrm{H}), 2.59(\mathrm{t}, J=7.0 \mathrm{~Hz}, 4 \mathrm{H}), 2.51(\mathrm{t}, J=6.6 \mathrm{~Hz}, 4 \mathrm{H}), 1.93$ (quint, $J=6.7 \mathrm{~Hz}, 4 \mathrm{H}), 1.67-1.78(8 \mathrm{H}), 0.93(\mathrm{~s}, 18 \mathrm{H}), 0.09(\mathrm{~s}, 12 \mathrm{H})$ ppm. ${ }^{13} \mathrm{C}$ NMR $(150 \mathrm{MHz}): \delta=138.5,135.2,128.4,127.60,127.56$, 125.2, 125.0, 95.4, 94.7, 79.2, 79.1, 73.0, 68.8, 62.7, 32.0, 28.9, 26.0, 25.1, 19.5, 18.4, 16.6, -5.3 ppm. FTIR: $\bar{v}=2928,2855,2209,1487$, $1253,1102,900,834,774,733,696 \mathrm{~cm}^{-1}$. HRMS (ESI): calcd. for $\mathrm{C}_{54} \mathrm{H}_{74} \mathrm{O}_{4} \mathrm{Si}_{2} \mathrm{Na}[\mathrm{M}+\mathrm{Na}]^{+}$865.5023; found 865.5006.

5,5'-[4,5-Bis(5-benzyloxypent-1-ynyl)-1,2-phenylene]dipent4-ynyl Dibenzoate (3b): Yield: $127 \mathrm{mg}, 0.16 \mathrm{mmol}, 64 \%$. Mobile phase: $15 \%$ ethyl acetate/hexane. $R_{\mathrm{f}}=0.39(20 \%$ ethyl acetate/ hexane). ${ }^{1} \mathrm{H}$ NMR (600 MHz): $\delta=8.04-8.10(4 \mathrm{H}), 7.54-7.60$ (2 $\mathrm{H}), 7.42-7.47$ (4 H), 7.39-7.41 (2 H), 7.33-7.38 (8 H), 7.27-7.31 (2 $\mathrm{H}), 4.55(\mathrm{~s}, 4 \mathrm{H}), 4.53(\mathrm{t}, J=6.3 \mathrm{~Hz}, 4 \mathrm{H}), 3.66(\mathrm{t}, J=6.2 \mathrm{~Hz}, 4 \mathrm{H})$, $2.70(\mathrm{t}, J=7.0 \mathrm{~Hz}, 4 \mathrm{H}), 2.60(\mathrm{t}, J=7.0 \mathrm{~Hz}, 4 \mathrm{H}), 2.11$ (quint, $J=$ $6.6 \mathrm{~Hz}, 4 \mathrm{H}$ ), 1.94 (quint, $J=6.6 \mathrm{~Hz}, 4 \mathrm{H}) \mathrm{ppm} .{ }^{13} \mathrm{C}$ NMR $(150$ MHz): $\delta=166.5,138.5,135.3,133.0,130.2,129.6,128.38,128.36$, 127.60, 127.56, 125.3, 124.9, 95.0, 93.9, 79.6, 79.1, 73.0, 68.7, 63.7, $28.9,28.0,16.7,16.6$ ppm. FTIR: $v=3675,2988,2972,2901,2229$,
1716, 1451, 1394, 1269, 1107, 1068, 1027, $900 \mathrm{~cm}^{-1}$. HRMS (ESI): calcd. for $\mathrm{C}_{54} \mathrm{H}_{50} \mathrm{O}_{6} \mathrm{Na}[\mathrm{M}+\mathrm{Na}]^{+}$817.3505; found 817.3503.

1,2-Bis(5-benzyloxypent-1-ynyl)-4,5-bis(2-trimethylsilylethynyl)benzene (3c): Yield: $98 \mathrm{mg}, 0.16 \mathrm{mmol}, 64 \%$. Mobile phase: $6 \%$ ethyl acetate/hexane. $R_{\mathrm{f}}=0.41$ (10\% ethyl acetate/hexane). ${ }^{1} \mathrm{H}$ NMR $(600 \mathrm{MHz}): \delta=7.48(\mathrm{~s}, 2 \mathrm{H}), 7.28-7.41(10 \mathrm{H}), 4.56(\mathrm{~s}, 4$ $\mathrm{H}), 3.66(\mathrm{t}, J=6.1 \mathrm{~Hz}, 4 \mathrm{H}), 2.61(\mathrm{t}, J=7.0 \mathrm{~Hz}, 4 \mathrm{H}), 1.94$ (quint, $J=6.4 \mathrm{~Hz}, 4 \mathrm{H}), 0.32(\mathrm{~s}, 18 \mathrm{H}) \mathrm{ppm} .{ }^{13} \mathrm{C} \mathrm{NMR}(150 \mathrm{MHz}): \delta=$ 138.5, 135.6, 128.4, 127.62, 127.61, 126.0, 124.5, 102.4, 100.0, 95.6, 79.0, 73.0, 68.7, 28.9, 16.6, 0.01 ppm. FTIR: $\bar{v}=2956,2856,2226$, 2155, 1481, 1454, 1249, 1103, 1077, 1028, 839, 758, 731, 696, 635 $\mathrm{cm}^{-1}$. HRMS (ESI): calcd. for $\mathrm{C}_{40} \mathrm{H}_{46} \mathrm{O}_{2} \mathrm{Si}_{2} \mathrm{Na}[\mathrm{M}+\mathrm{Na}]^{+}$637.2934; found 637.2922.

1,2-Bis(5-benzyloxypent-1-ynyl)-4,5-bis(2-phenylethynyl)benzene (3d): Yield: $130 \mathrm{mg}, 0.208 \mathrm{mmol}, 83 \%$. Mobile phase: step gradient $5-10 \%$ ethyl acetate/hexane. $R_{\mathrm{f}}=0.32(10 \%$ ethyl acetate/hexane). ${ }^{1} \mathrm{H}$ NMR (600 MHz): $\delta=7.57-7.63(6 \mathrm{H}), 7.34-7.42$ (14 H), 7.29-7.34 (2 H), $4.58(\mathrm{~s}, 4 \mathrm{H}), 3.69(\mathrm{t}, J=6.1 \mathrm{~Hz}, 4 \mathrm{H}), 2.64$ $(\mathrm{t}, J=7.0 \mathrm{~Hz}, 4 \mathrm{H}), 1.97$ (quint, $J=6.6 \mathrm{~Hz}, 4 \mathrm{H}$ ) ppm. ${ }^{13} \mathrm{C} \mathrm{NMR}$ $(150 \mathrm{MHz}): \delta=138.5,135.1,131.7,128.7,128.5,128.4,127.63$, $127.61,125.9,124.6,123.1,95.6,94.9,87.6,79.2,73.0,68.7,28.9$, 16.6 ppm. FTIR: $\bar{v}=2856,2224,1597,1497,1364,1102,1076,1027$, 899, 754, 734, $688 \mathrm{~cm}^{-1}$. HRMS (ESI): calcd. for $\mathrm{C}_{46} \mathrm{H}_{38} \mathrm{O}_{6} \mathrm{Na}[\mathrm{M}+$ $\mathrm{Na}]^{+}$645.2770; found 645.2759.

1,2-Bis(5-benzyloxypent-1-ynyl)-4,5-bis[2-(4-methoxyphenyl) ethynyl]benzene (3e): Yield: $135 \mathrm{mg}, 0.198 \mathrm{mmol}, 79 \%$. Mobile phase: $15 \%$ ethyl acetate/hexane. $R_{\mathrm{f}}=0.37(20 \%$ ethyl acetate/ hexane). ${ }^{1} \mathrm{H}$ NMR $(600 \mathrm{MHz}): \delta=7.49-7.56(6 \mathrm{H}), 7.34-7.42(8 \mathrm{H})$, 7.28-7.38 (2 H), 6.88-6.93 (4 H), $4.56(\mathrm{~s}, 4 \mathrm{H}), 3.86(\mathrm{~s}, 6 \mathrm{H}), 3.68(\mathrm{t}$, $J=6.1 \mathrm{~Hz}, 4 \mathrm{H}), 2.62(\mathrm{t}, J=7.0 \mathrm{~Hz}, 4 \mathrm{H}), 1.95$ (quint, $J=6.6 \mathrm{~Hz}, 4$ H) ppm. ${ }^{13} \mathrm{C}$ NMR $(150 \mathrm{MHz}): \delta=159.2,138.5,134.8,133.2,128.4$, 127.62, 127.59, 125.5, 124.7, 115.3, 114.1, 95.2, 94.9, 86.6, 79.3, 73, 68.7, 55.3, 28.9, 16.6 ppm. FTIR: $\bar{v}=2932,2857,2209,1604,1568$, $1511,1289,1246,1172,1141,1104,1027,899,829,734,696 \mathrm{~cm}^{-}$ 1. HRMS (ESI): calcd. for $\mathrm{C}_{48} \mathrm{H}_{42} \mathrm{O}_{4} \mathrm{Na}[\mathrm{M}+\mathrm{Na}]^{+}$705.2981; found 705.2974 .

1,2-Bis(5-benzyloxypent-1-ynyl)-4,5-bis[2-(4-fluorophenyl) ethynyl]benzene (3f): Yield: $122 \mathrm{mg}, 0.185 \mathrm{mmol}, 74 \%$. Mobile phase: $8 \%$ ethyl acetate/hexane. $R_{\mathrm{f}}=0.36(10 \%$ ethyl acetate/ hexane). ${ }^{1} \mathrm{H}$ NMR $(600 \mathrm{MHz}): \delta=7.52-7.58(6 \mathrm{H}), 7.34-7.42(8 \mathrm{H})$, $7.28-7.34(2 \mathrm{H}), 7.05-7.11(4 \mathrm{H}), 4.57(\mathrm{~s}, 4 \mathrm{H}), 3.68(\mathrm{t}, J=6.1 \mathrm{~Hz}, 4$ $\mathrm{H}), 2.63(\mathrm{t}, J=7.0 \mathrm{~Hz}, 4 \mathrm{H}), 1.96$ (quint, $J=6.6 \mathrm{~Hz}, 4 \mathrm{H}) \mathrm{ppm} .{ }^{13} \mathrm{C}$ NMR (150 MHz): $\delta=162.8\left(\mathrm{~d}, J_{\mathrm{C}, \mathrm{F}}=250.5 \mathrm{~Hz}\right), 138.5,135.0,133.5$ $\left(\mathrm{d}, J_{\mathrm{C}, \mathrm{F}}=8.6 \mathrm{~Hz}\right), 128.4,127.61,127.60,126.0,124.3,119.2\left(\mathrm{~d}, J_{\mathrm{C}, \mathrm{F}}\right.$ $=4.4 \mathrm{~Hz}), 115.8\left(\mathrm{~d}, J_{\mathrm{C}, \mathrm{E}}=22.5 \mathrm{~Hz}\right), 95.7,93.7,87.3,79.1,73.0,68.7$, 28.9, 16.6 ppm. FTIR: $v=2926,2856,2225,1600,1567,1507,1227$, $1155,1102,899,833,792,734,696 \mathrm{~cm}^{-1}$. HRMS (ESI): calcd. for $\mathrm{C}_{46} \mathrm{H}_{36} \mathrm{~F}_{2} \mathrm{O}_{2} \mathrm{Na}[\mathrm{M}+\mathrm{Na}]^{+}$681.2581; found 681.2567.

5,5'-\{4,5-Bis[6-(tert-butyldimethylsilyloxy)hex-1-ynyl]-1,2phenylene\}dipent-4-ynyl Dibenzoate (3g): Yield: $149 \mathrm{mg}, 0.170$ mmol, $68 \%$. Mobile phase: $6 \%$ ethyl acetate/hexane. $R_{\mathrm{f}}=0.36$ (10\% ethyl acetate/hexane). ${ }^{1} \mathrm{H}$ NMR (600 MHz): $\delta=8.03-8.08$ (4 H), 7.34-7.42 (8 H), 7.52-7.59 (2 H), 7.38-7.47 (6 H), $4.50(\mathrm{t}, J$ $=6.3 \mathrm{~Hz}, 4 \mathrm{H}), 3.68(\mathrm{t}, J=6.0 \mathrm{~Hz}, 4 \mathrm{H}), 2.67(\mathrm{t}, J=6.8 \mathrm{~Hz}, 4 \mathrm{H})$, $2.50(\mathrm{t}, J=6.5 \mathrm{~Hz}, 4 \mathrm{H}), 2.09$ (quint, $J=6.5 \mathrm{~Hz}, 4 \mathrm{H}), 1.65-1.77(8$ $\mathrm{H}), 0.92(\mathrm{~s}, 18 \mathrm{H}), 0.07(\mathrm{~s}, 12 \mathrm{H}) \mathrm{ppm} .{ }^{13} \mathrm{C} \mathrm{NMR}(150 \mathrm{MHz}): \delta=$ $166.5,135.2,132.9,130.2,129.6,128.3,125.4,124.8,95.6,93.8,79.6$, 79.0, 63.7, 62.6, 31.9, 28.0, 26.0, 25.1, 19.5, 18.3, 16.6, -5.3 ppm. FTIR: $\bar{v}=2951,2928,2856,2231,1719,1270,1107,1070,834,709$ $\mathrm{cm}^{-1}$. HRMS (ESI): calcd. for $\mathrm{C}_{54} \mathrm{H}_{70} \mathrm{O}_{6} \mathrm{Si}_{2} \mathrm{Na}[\mathrm{M}+\mathrm{Na}]^{+} 893.4609$; found 893.4631 .

1,2-Bis[2-(4-methoxyphenyl)ethynyl]-4,5-bis(2-trimethylsilylethynyl)benzene (3h): Yield: $76 \mathrm{mg}, 0.143 \mathrm{mmol}, 57 \%$. Mo- 
bile phase: $4 \%$ ethyl acetate/hexane. $R_{\mathrm{f}}=0.38(10 \%$ ethyl acetate/hexane). ${ }^{1} \mathrm{H}$ NMR (600 MHz): $\delta=7.66$ (s, $\left.2 \mathrm{H}\right), 7.47-7.55(4$ $\mathrm{H}), 6.86-6.93$ (4 H), 3.85 (s, $6 \mathrm{H}), 0.31$ (s, $18 \mathrm{H}) \mathrm{ppm} .{ }^{13} \mathrm{C}$ NMR (150 MHz): $\delta=160.0,135.2,133.2,125.6,124.8,115.1,114.1,102.4$, 100.4, 95.7, 86.4, 55.3, 0.0 ppm. FTIR: $v=2959,2213,2156,1605$, 1513, 1290, 1246, 1167, 1031, $828 \mathrm{~cm}^{-1}$. HRMS (EI): calcd. for $\mathrm{C}_{34} \mathrm{H}_{34} \mathrm{O}_{2} \mathrm{Si}_{2}[\mathrm{M}]^{+}$530.2097; found 530.2093.

1,2-Bis\{2-[4-(dibutylamino)phenyl]ethynyl\}-4,5-bis[2-(2-pyridyl)ethynyl]benzene (3i): Yield: $134 \mathrm{mg}, 0.183 \mathrm{mmol}, 73 \%$. Mobile phase: $35 \%$ ethyl acetate/hexane. $R_{\mathrm{f}}=0.35$ (40\% ethyl acetate/hexane). ${ }^{1} \mathrm{H}$ NMR $(600 \mathrm{MHz}): \delta=8.64-8.70(2 \mathrm{H}), 7.78(\mathrm{~s}$, $2 \mathrm{H}), 7.73(\mathrm{~d}, J=7.6 \mathrm{~Hz}, 2 \mathrm{H}), 7.67(\mathrm{td}, J=7.8,1.9 \mathrm{~Hz}, 2 \mathrm{H}), 7.45$ $(\mathrm{d}, J=8.7 \mathrm{~Hz}, 2 \mathrm{H}), 7.23-7.28(2 \mathrm{H}), 6.61(\mathrm{~d}, J=8.7 \mathrm{~Hz}, 4 \mathrm{H}), 3.32$ $(\mathrm{t}, J=7.9 \mathrm{~Hz}, 8 \mathrm{H}$ ), 1.61 (quint, $J=7.8 \mathrm{~Hz}, 8 \mathrm{H}$ ), 1.39 (sext, $J=7.6$ $\mathrm{Hz}, 8 \mathrm{H}), 0.99$ (t, $J=7.3 \mathrm{~Hz}, 12 \mathrm{H}) \mathrm{ppm} .{ }^{13} \mathrm{C}$ NMR $(150 \mathrm{MHz}): \delta$ $=150.1,148.3,143.5,136.1,134.8,133.3,127.8,126.7,123.4,122.9$, $111.2,108.5,98.0,93.9,87.4,86.0,50.7,29.4,20.3,14.0$ ppm.

\section{Supporting Information}

${ }^{1} \mathrm{H}$ and ${ }^{13} \mathrm{C}$ NMR spectra for all compounds are presented following the References.

Acknowledgments - We are grateful for financial support from the National Science Foundation (NSF) (EPS 10040 94). Preliminary experiments were conducted by Mitch Trauernicht.

\section{References}

[1] R. Chinchilla, C. Najera, Chem. Rev. 2007, 107, 874; R. Chinchilla, C. Najera, Chem. Soc. Rev. 2011, 40, 5084.

[2] a) E. L. Spitler, L. D. Shirtcliff, M. M. Haley, J. Org. Chem. 2007, 72, 86; b) J. A. Marsden, J. Miller, L. D. Shirtcliff, M. M. Haley, J. Am. Chem. Soc. 2005, 127, 2464, and references therein; c) K. Ohta, S. Yamada, K. Kamada, A. D. Slepkov, F. A. Hegmann, R. R. Tykwinski, L. D. Shirtcliff, M. M. Haley, P. Sałek, F. Gel'mukhanov, H. Ågren, J. Phys. Chem. A 2011, 115, 105.
[3] a) W. Tao, S. Nesbitt, R. F. Heck, J. Org. Chem. 1990, 55, 63; b) I. A. Balova, V. N. Sorokoumov, S. N. Morozkina, O. V. Vinogradova, D. W. Knight, S. F. Vasilevsky, Eur. J. Org. Chem. 2005, 882; c) E. L. Spitler, M. M. Haley, Org. Biomol. Chem. 2008, 6, 1569.

[4] a) J. A. MacBride, K. Wade, Synth. Commun. 1996, 26, 2309; b) K. Tahara, T. Yoshimura, M. Ohno, M. Sonoda, Y. Tobe, Chem. Lett. 2007, 36, 838; c) J. Mack, P. Vogel, D. Jones, N. Kaval, A. Sutton, Org. Biomol. Chem. 2007, 5, 2448; d) E. Reisner, S. J. Lippard, Eur. J. Org. Chem. 2008, 156; e) C. M. Kane, T. B. Meyers, X. Yu, M. Gerken, M. Etzkorn, Eur. J. Org. Chem. 2011, 2969.

[5] a) T. Kamikawa, T. J. Hayashi, Org. Chem. 1998, 63, 8922; b) H. Y. Lee, A. Olasz, M. Pink, H. Park, D. Lee, Chem. Commun. 2011, 47, 481; c) X. Jiang, B. G. Park, J. A. Riddle, B. J. Zhang, M. Pink, D. Lee, Chem. Commun. 2008, 6028; d) M. Lu, Y. Pan, Z. Peng, Tetrahedron Lett. 2002, 43, 7903.

[6] a) N. A. Powell, S. D. Rychnovsky, Tetrahedron Lett. 1996, 37, 7901; b) R. A. Khera, M. Nawaz, H. Feist, A. Villinger, P. Langer, Synthesis 2012, 219.

[7] J. Lacour, D. Monchaud, G. Bernardinelli, F. Favarger, Org. Lett. 2001, 3, 1407.

[8] a) J. A. Marsden, M. M. Haley in Metal-Catalyzed Cross-Coupling Reactions (ed. A. de Meijere, F. Diederich), 2nd ed., Wiley-VCH, Weinheim, 2004, pp. 317-394; b) K. Sonogashira in Metal-Catalyzed Cross-Coupling Reactions (ed. F. Diederich, P. J. Stang), Wiley-VCH, Weinheim, 1998, pp. 203-230.

[9] a) B. M. Trost, A. S. Tasker, G. Ruther, A. Brandes, J. Am. Chem. Soc. 1991, 113, 670; b) N. Chernyak, V. Gevorgyan, J. Am. Chem. Soc. 2008, 130, 5636.

[10] a) I. Larrosa, C. Somoza, A. Banquy, S. M. Goldup, Org. Lett. 2011, 13, 146; b) C.-G. Dong, Q.-S. Hu, J. Am. Chem. Soc. 2005, 127, 10006; c) D. J. Sinclair, M. S. Sherburn, J. Org. Chem. 2005, 70, 3730. 


\section{SUPPORTING INFORMATION}

DOI: 10.1002/ejoc.201200079

Title: Regioselective Synthesis of Tetraalkynylarenes by Consecutive Dual Sonogashira Coupling Reactions of the Bis(triflate) of 4,5-Diiodobenzene-1,2-diol

Author(s): Thomas J. Fisher and Patrick H. Dussault* 
${ }^{1} \mathrm{H}$ and ${ }^{13} \mathrm{C}$ Spectra:

1,2-diodo-4,5-dimethoxybenzene

1,2-dihydroxy-4,5-dimethoxybenzene

4,5-Diiodo-1,2-phenylene bistrifluoromethanesulfonate (1)

4,5-Bis(5-benzyloxypent-1-yn-1-yl)-1,2-phenylene bistriflate (2a)

4,5-Bis(trifluoromethylsulfonyloxy)-1,2-phenylene)-bis(pent-4-yne-1-ol-4-yl, benzoate ester) (2b)

4,5-Bis(hexanenitrile-6-yl)-1,2-phenylene bistriflate (2c)

4,5-Bis (6-(tert-butyldimethylsilyloxy)hex-1-yn-1-yl)-1,2-phenylene bistriflate (2d) $\left({ }^{1} \mathrm{H},{ }^{13} \mathrm{C}\right)$

4,5-Bis(6-chlorohex-1-yn-1-yl)-1,2-phenylene bistriflate (2e)

4,5-Bis(2-triisopropylsilylethynyl)-1,2-phenylene bistriflate (2f)

4,5-Bis (2-(4-methoxyphenyl)ethynyl)-1,2-phenylene bistriflate (2g)

4,5-Bis(2-(pyridin-2-yl)ethynyl)-1,2-phenylene bistriflate (2h)

4,5-Bis (4-((tetrahydro-2H-pyran-2-yl)oxy)but-1-yn-1-yl)-1,2-phenylene bistriflate (2i)

4,5-Bis(2-methyl-3-butyn-1-ol-4-yl)-1,2-phenylene bistriflate (2j)

4,5-Bis(3,3-diethoxyprop-1-yn-1-yl)-1,2-phenylene bistriflate (2k)

(4,5-Bis(trifluoromethylsulfonyloxy)-1,2-phenylene)bis(prop-2-yne-1-ol-3-yl, benzoate ester) (2I)

4,5-Bis(3-benzyloxyprop-1-yn-1-yl)-1,2-phenylene bistriflate (2m)

4,5-Bis(5-benzyloxypent-1-yn-1-yl)-1,2-phenylene bis (6-tert-butyldimethylsilyloxyhex-1-yn-1-yl) (3a)

4,5-Bis(5-benzyloxypent-1-yn-1-yl)-1,2-phenylene-bis(pent-4-yne-1-ol-5-yl, benzoate ester) (3b)

4,5-Bis(5-benzyloxypent-1-yn-1-yl)-1,2-phenylene bis(2-trimethylsilylethyn-1-yl) (3c)

4,5-Bis(5-benzyloxypent-1-yn-1-yl)-1,2-phenylene bis(2-phenylethyn-1-yl) (3d)

4,5-Bis(5-benzyloxypent-1-yn-1-yl)-1,2-phenylene) bis(2-(4-methoxyphenyl) ethyn-1-yl (3e)

4,4'-((4,5-Bis(5-benzyloxypent-1-yn-1-yl)-1,2-phenylene)bis-2(4-fluorophenyl)ethyn-1-yl (3f)

(4,5-Bis(6-tert-butyldimethylsilyloxyhex-1-yn-1-yl)-1,2-phenylene) bis(pent-4-yne-1-ol-5-yl, benzoate ester) (3g)

4,5-Bis (2-(4-methoxyphenyl)ethyn-1-yl)-1,2-phenylene) bis (2-trimethylsilylethynyl-1-yl) (3h)

4,5-Bis(2-(4-pyridyl)ethynyl-1,2-phenylene)bis-2(4-(N,N'-dibutylanilino)ethyn-1-yl (3i) 


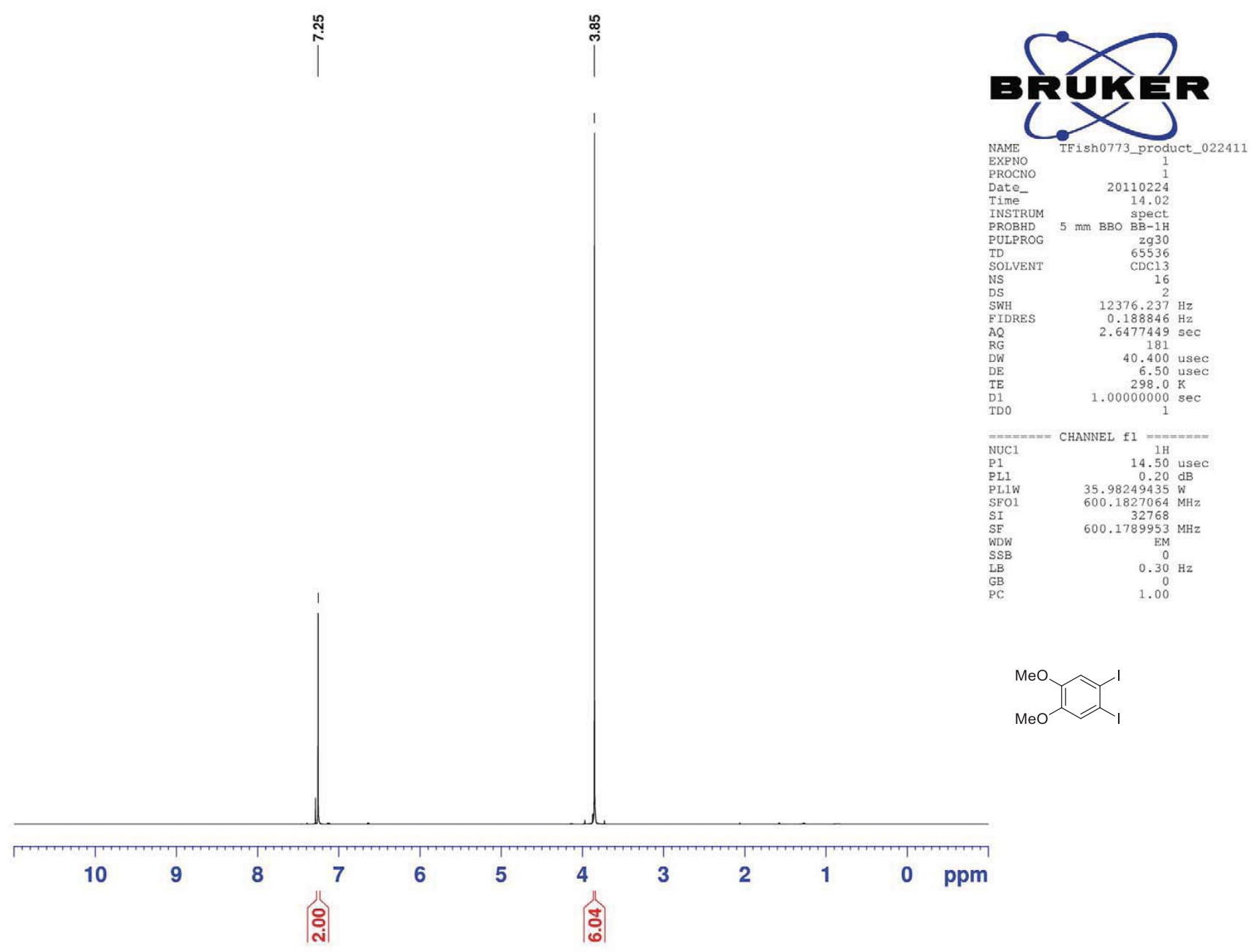




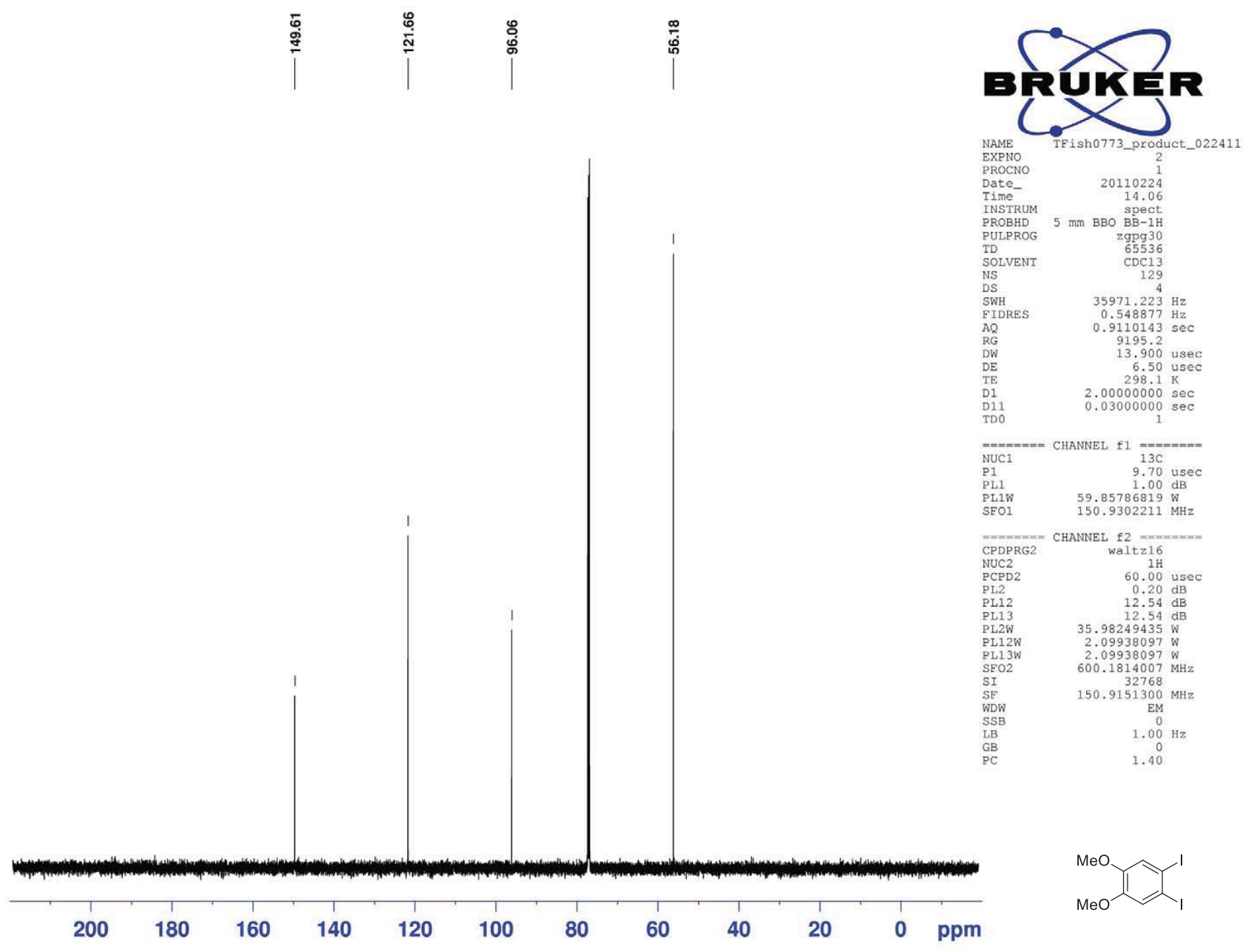




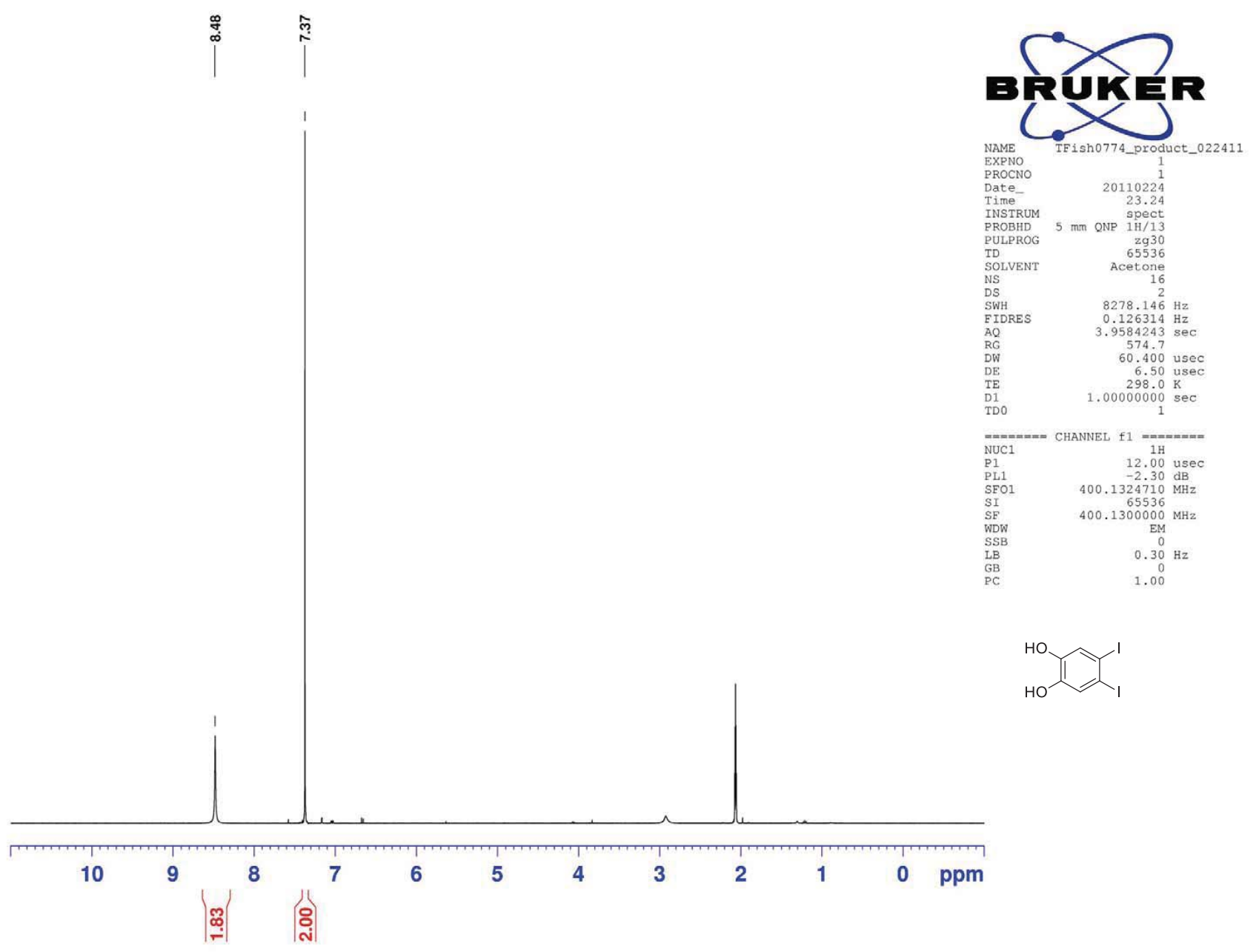




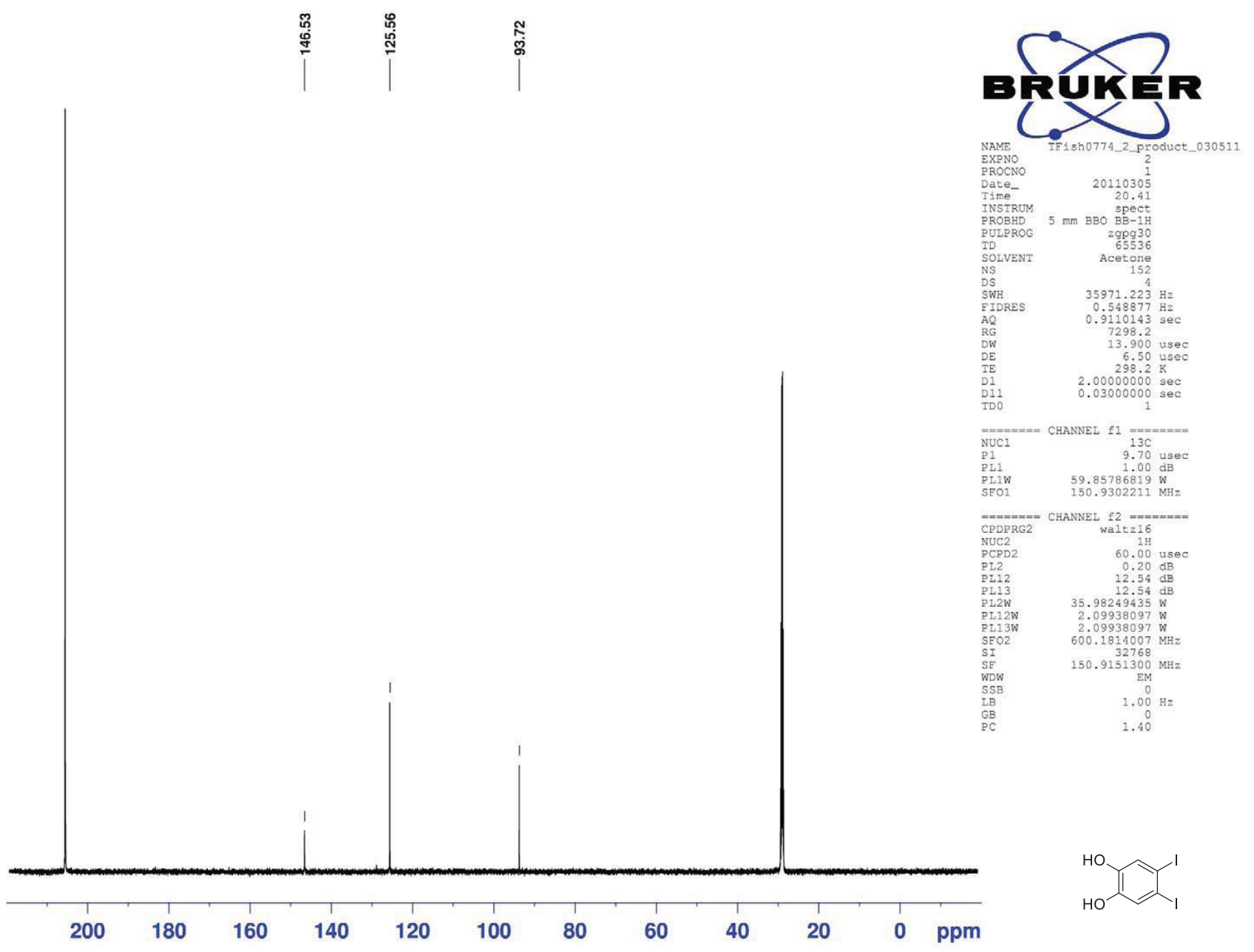




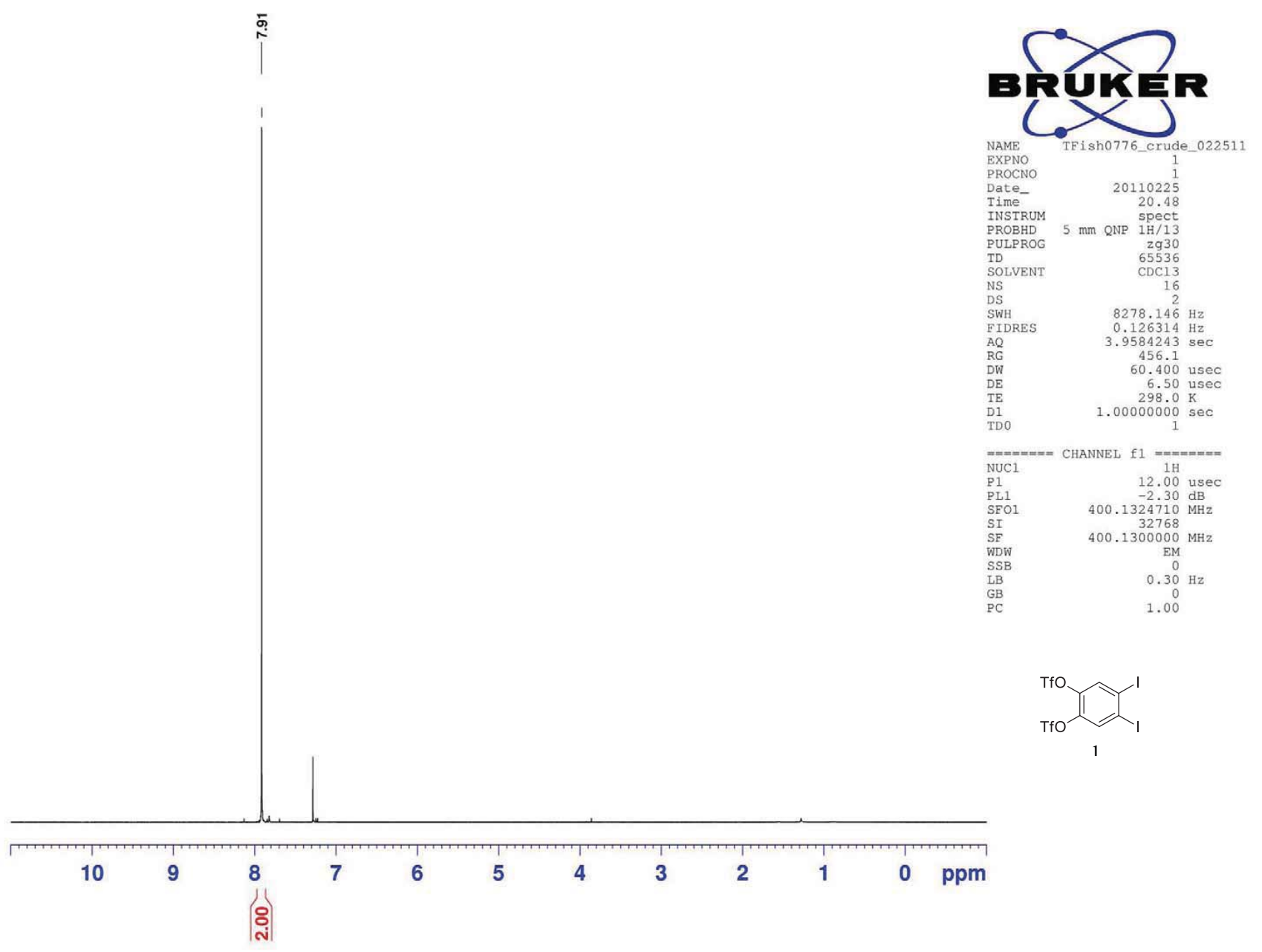




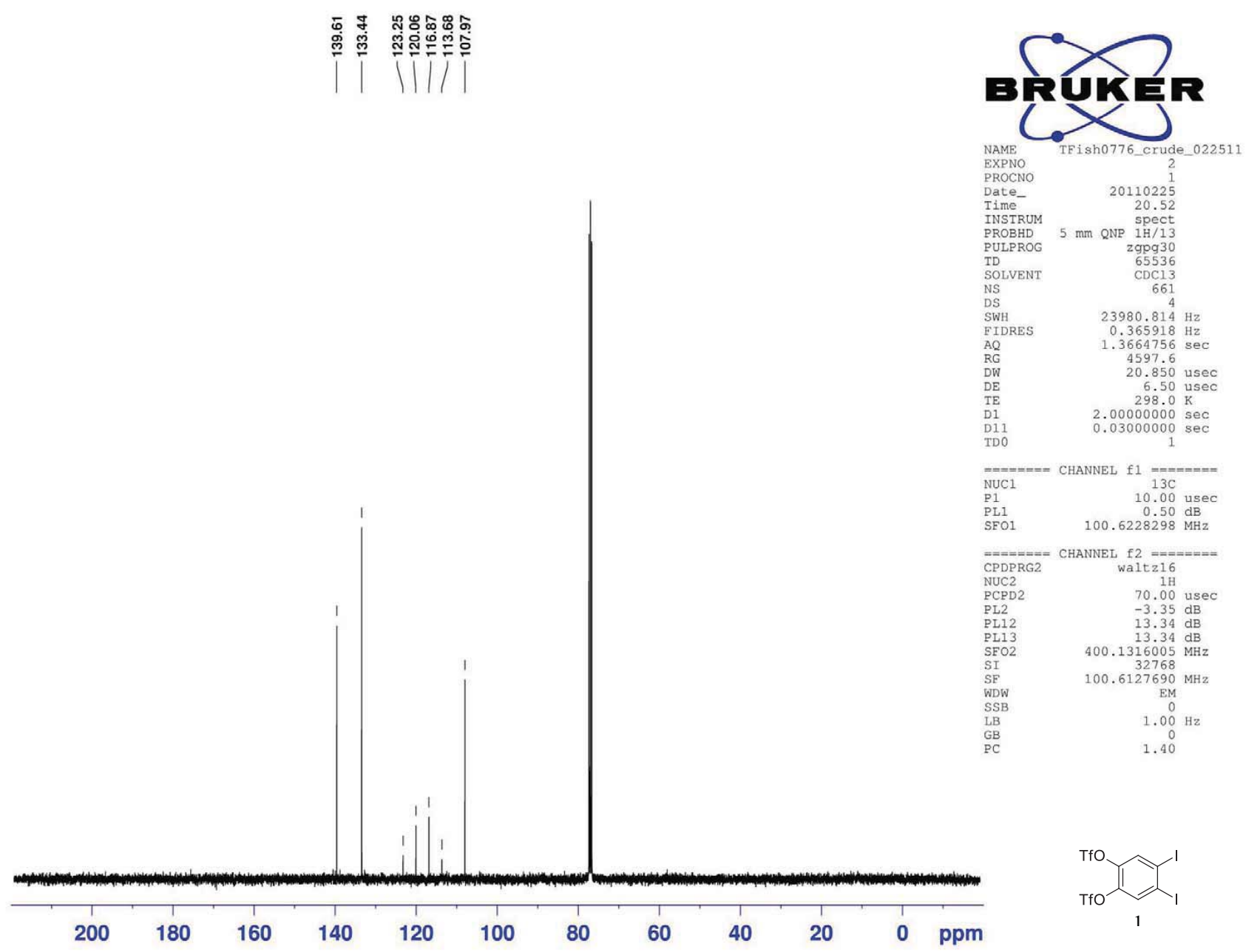




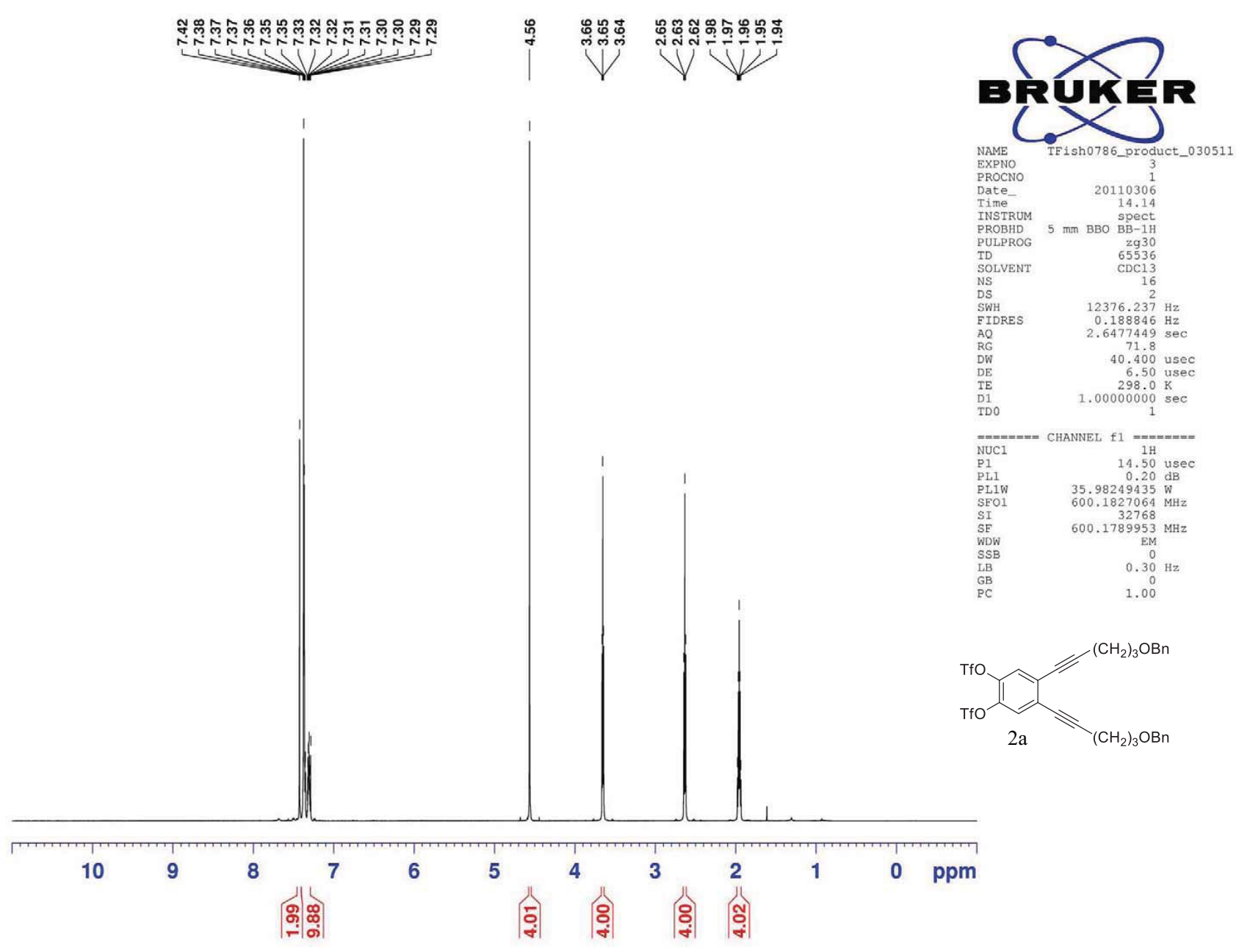




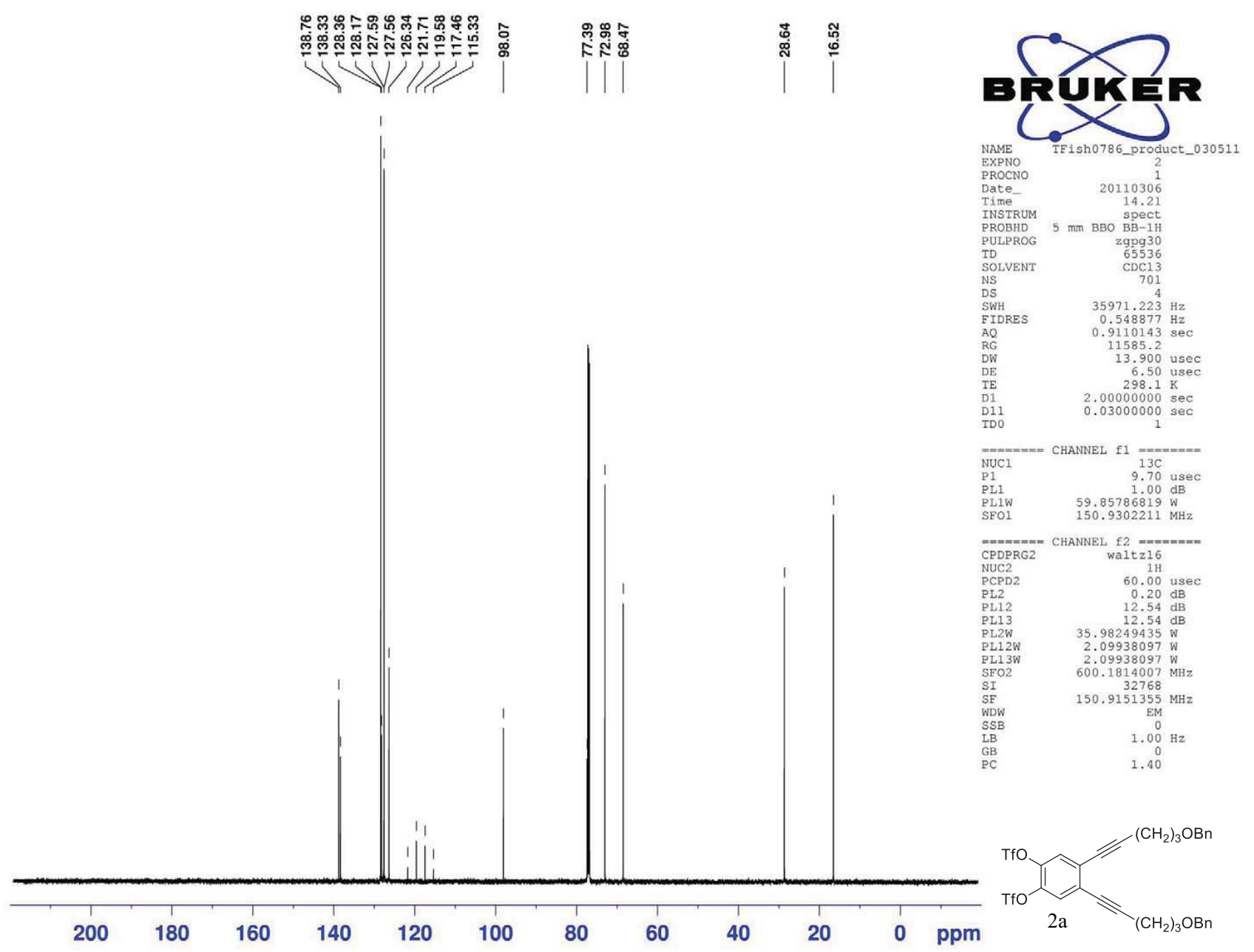




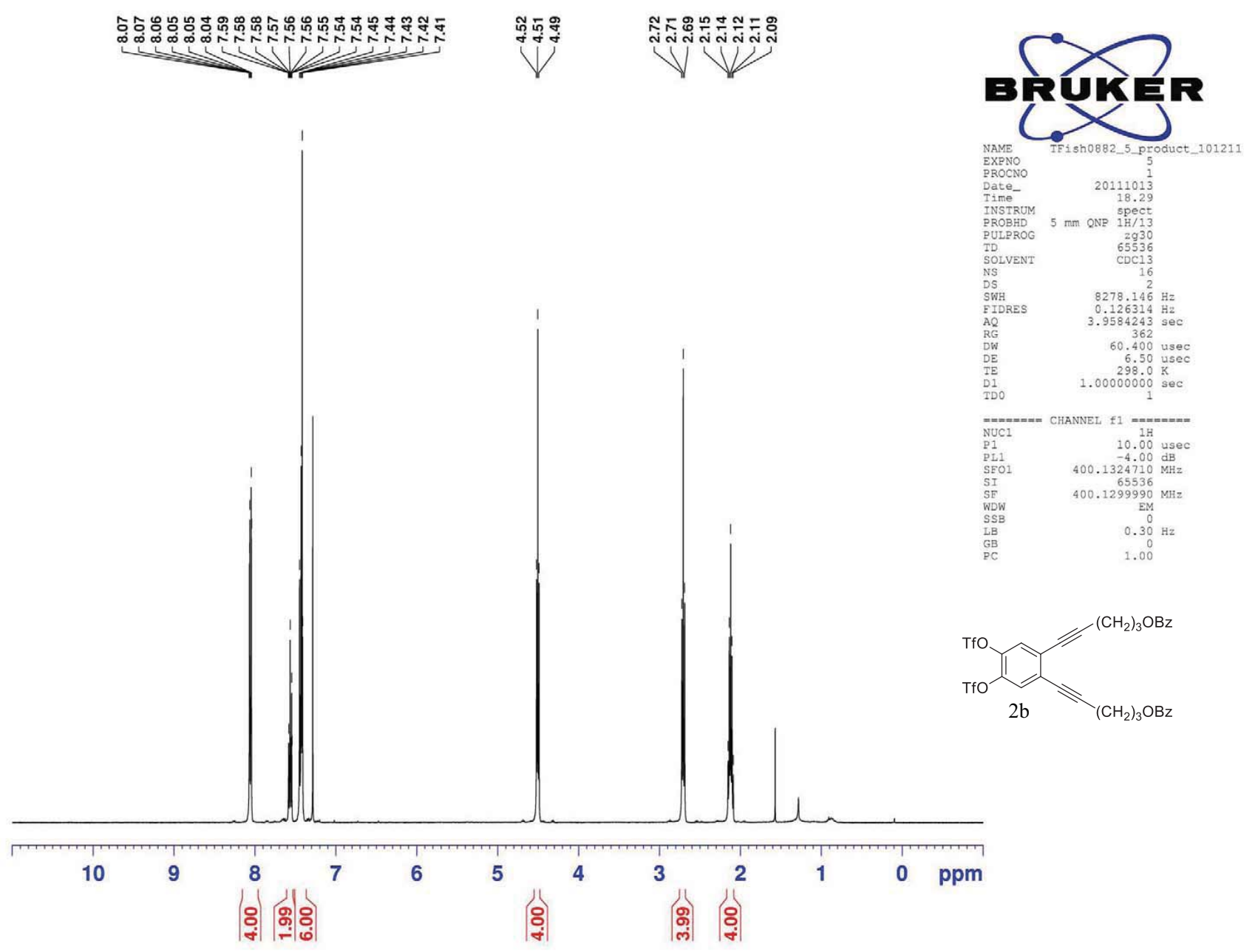




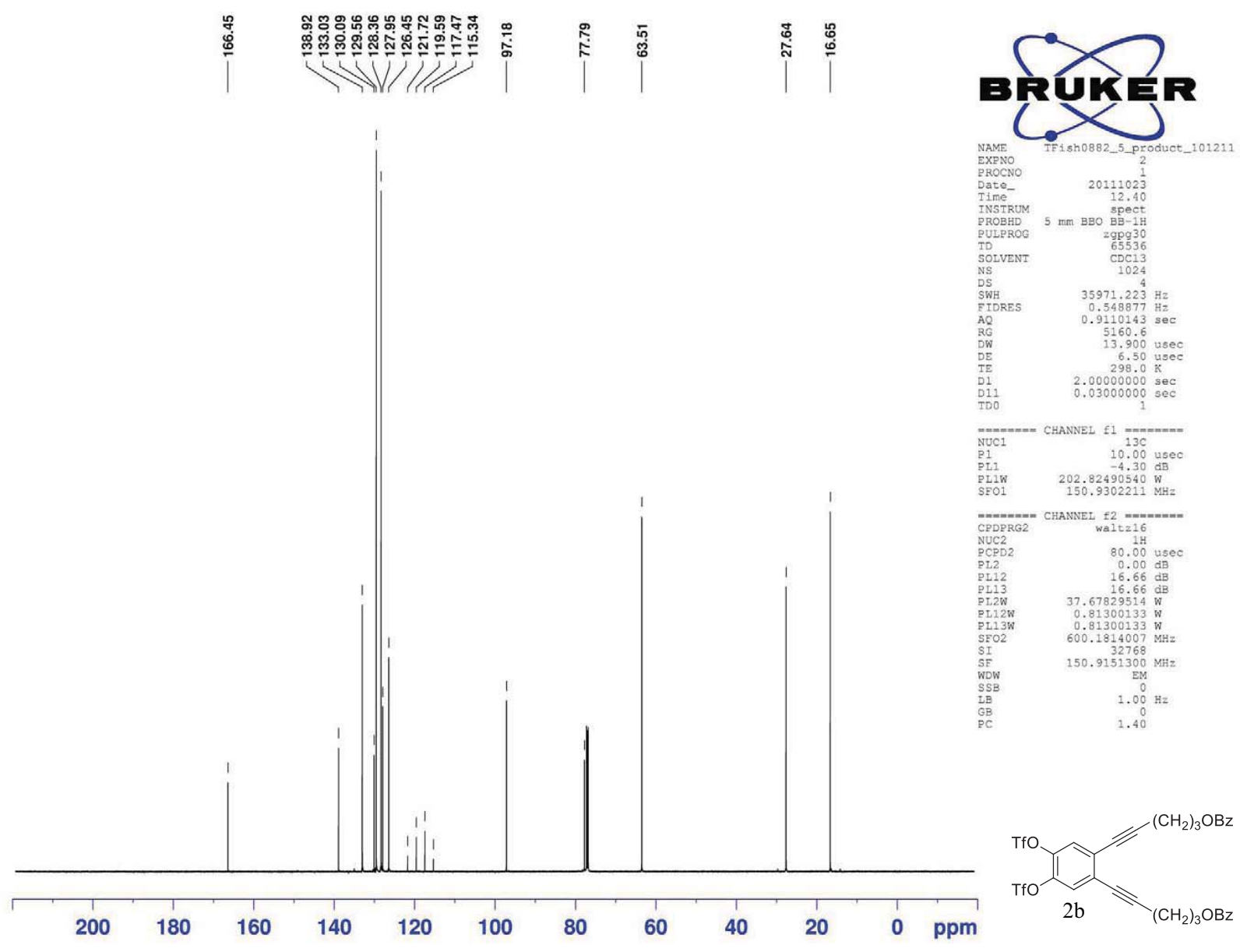




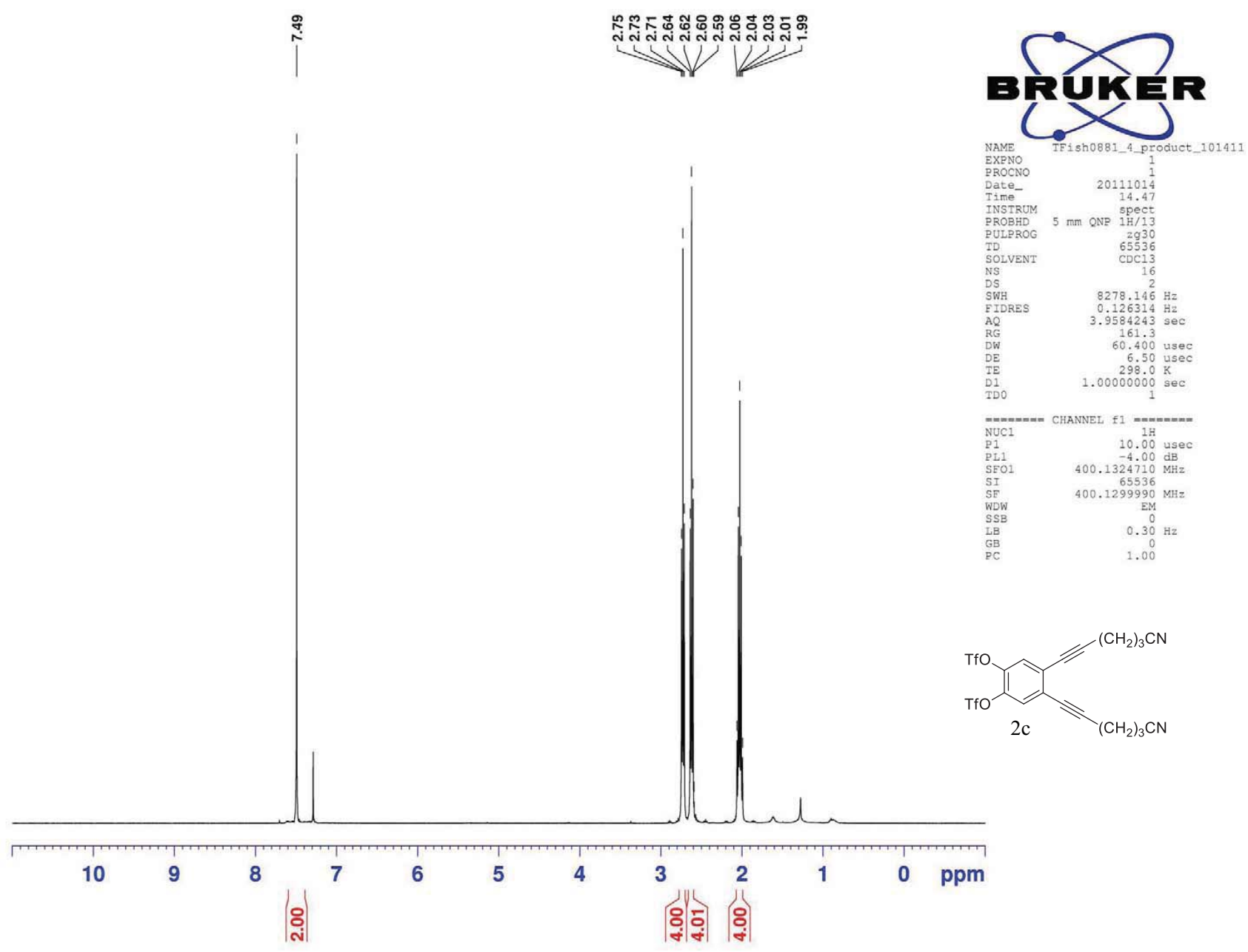




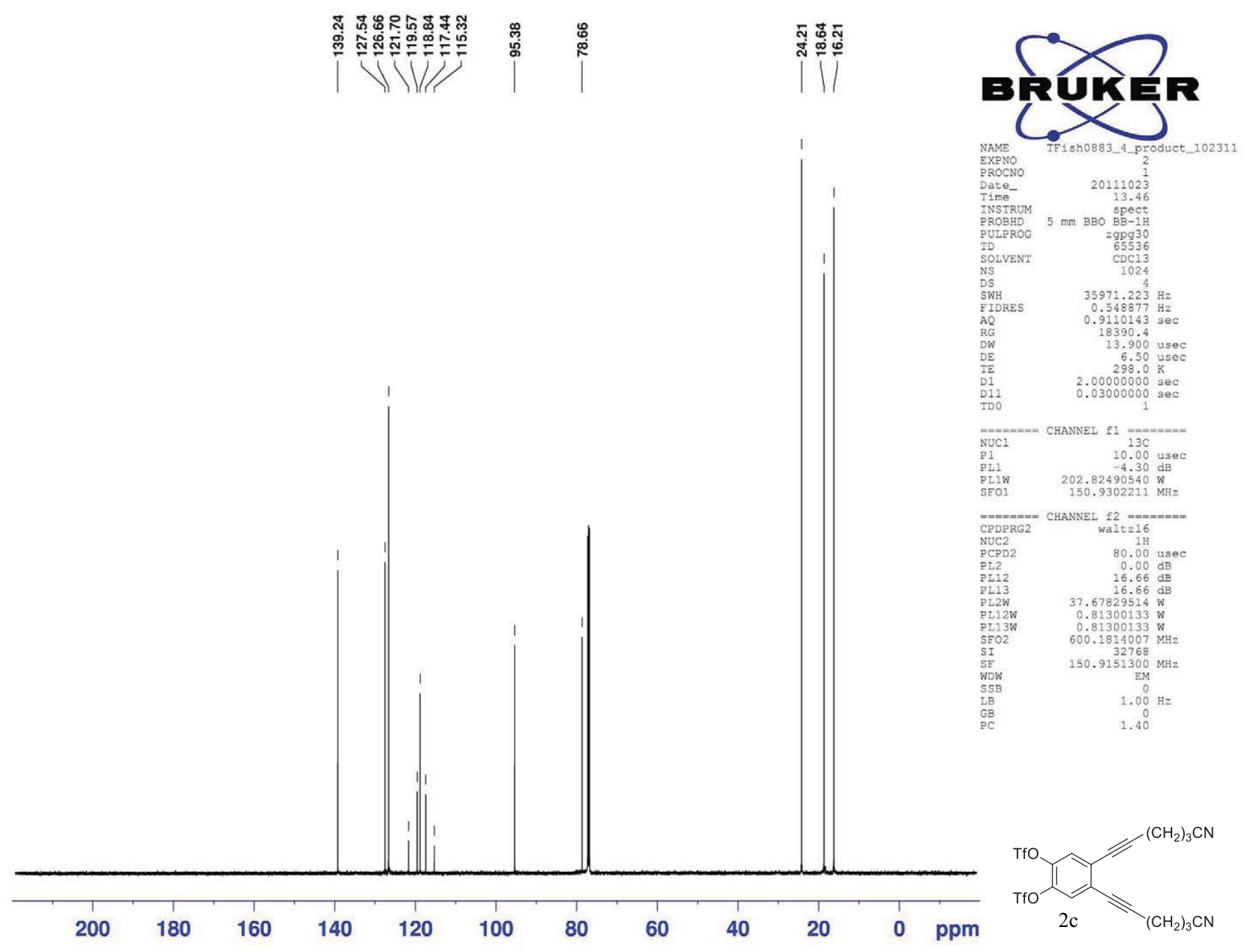




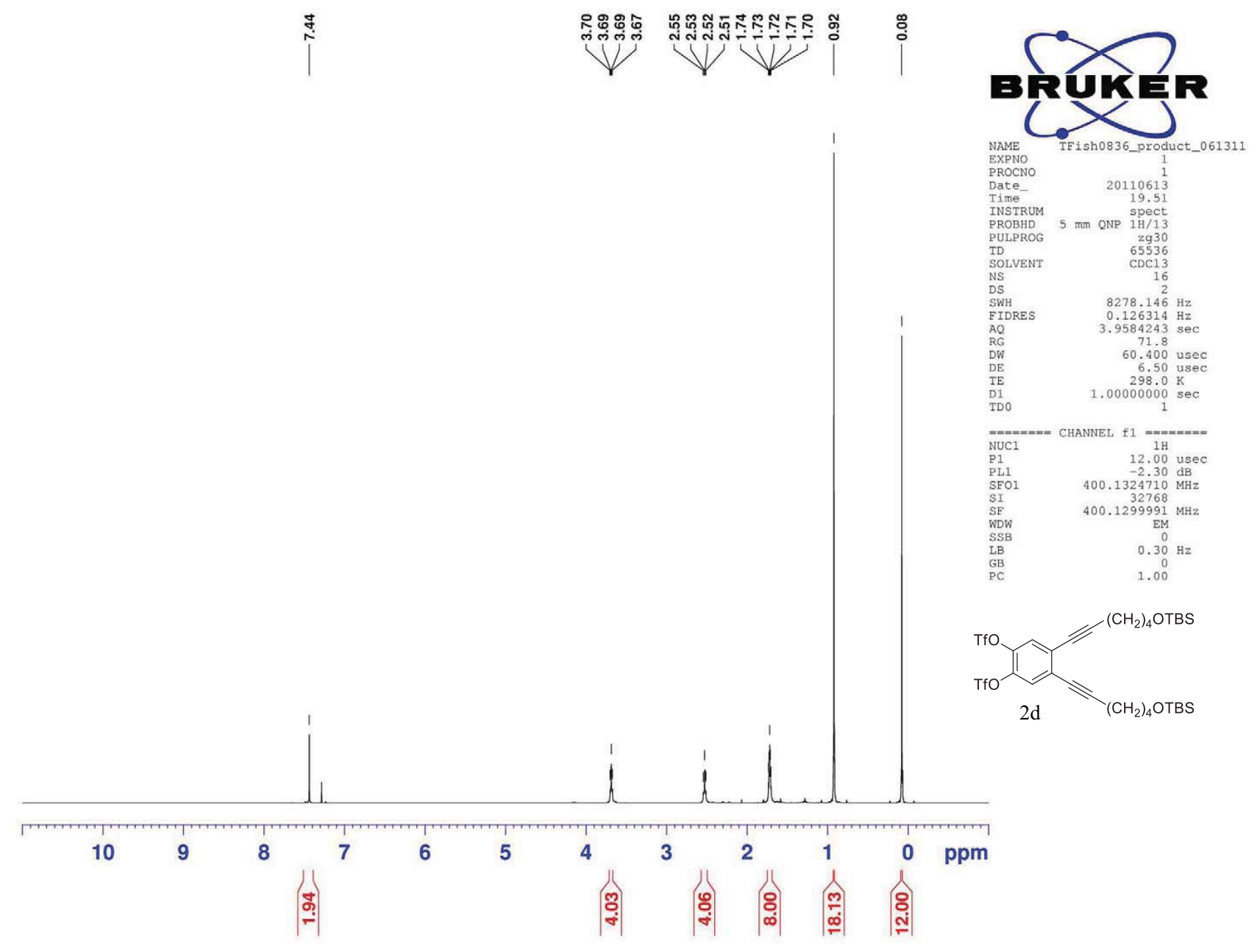




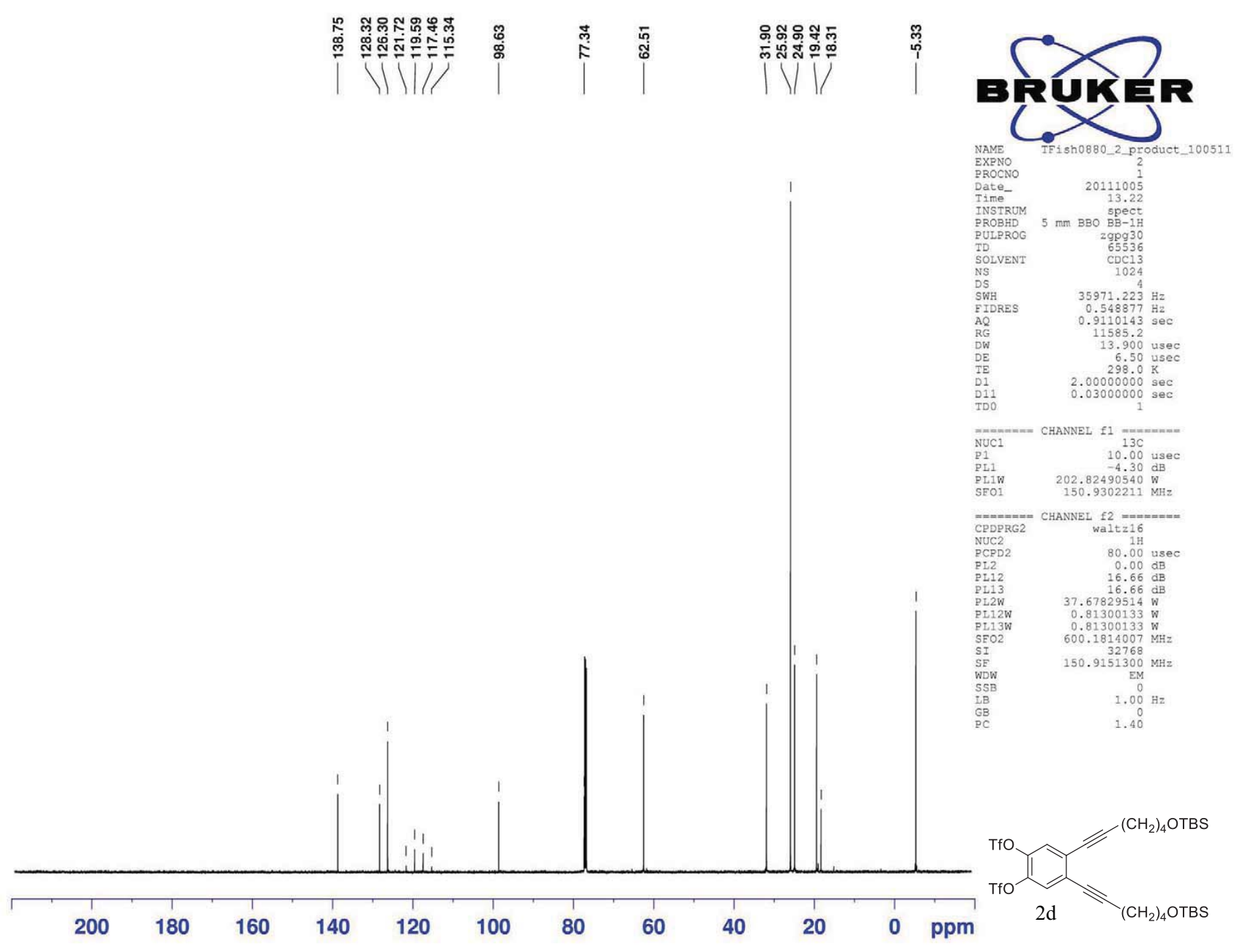




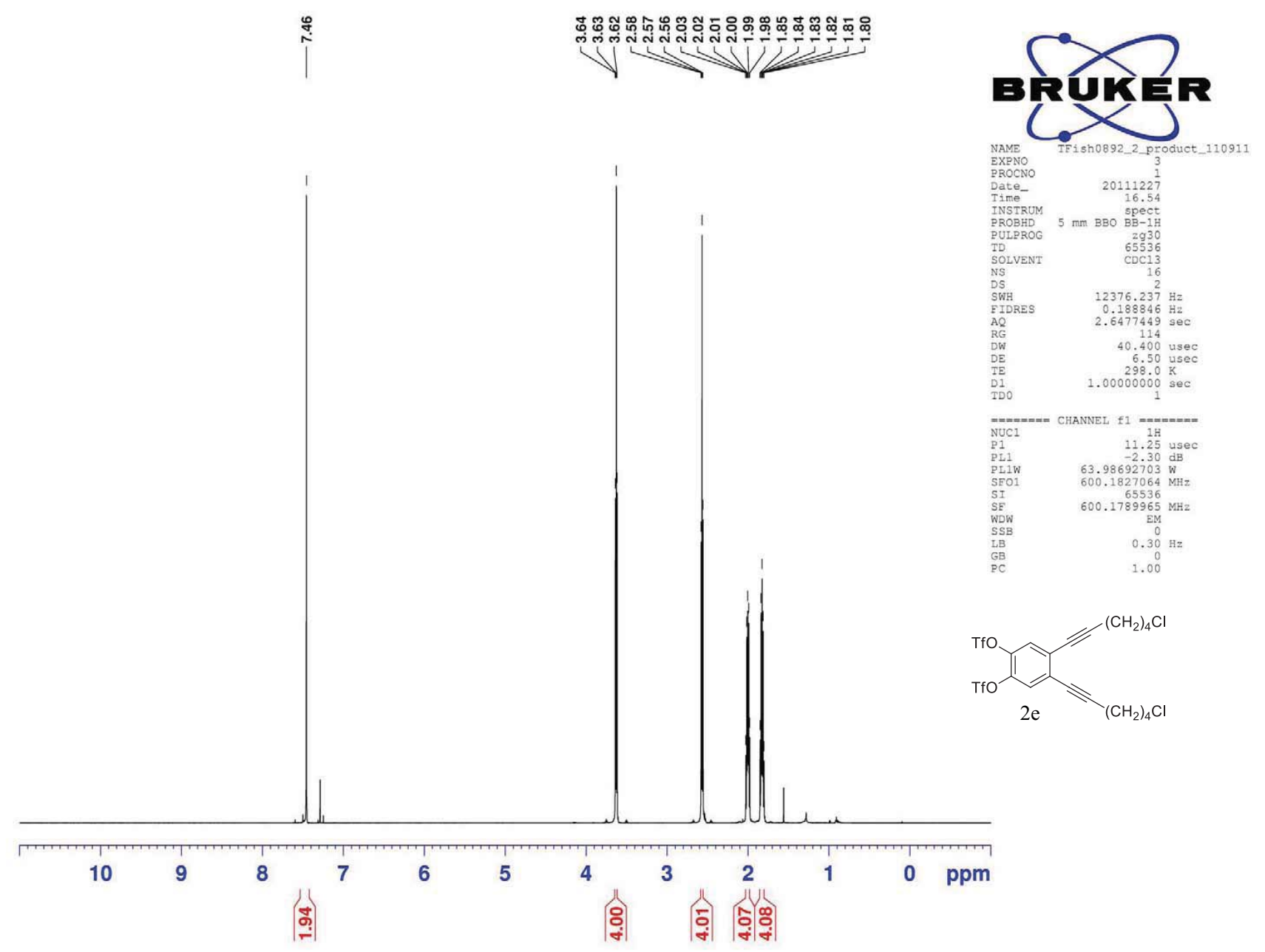




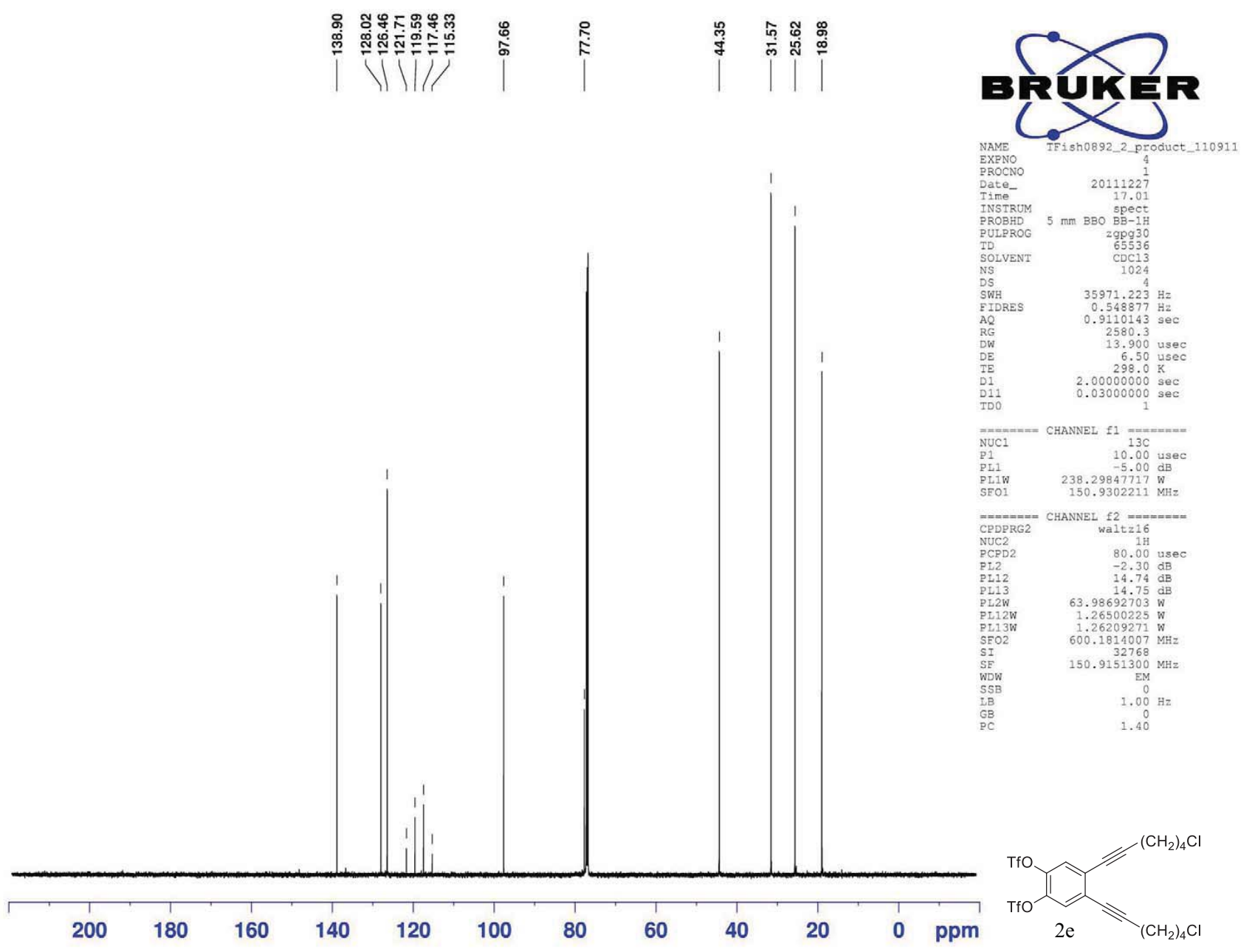




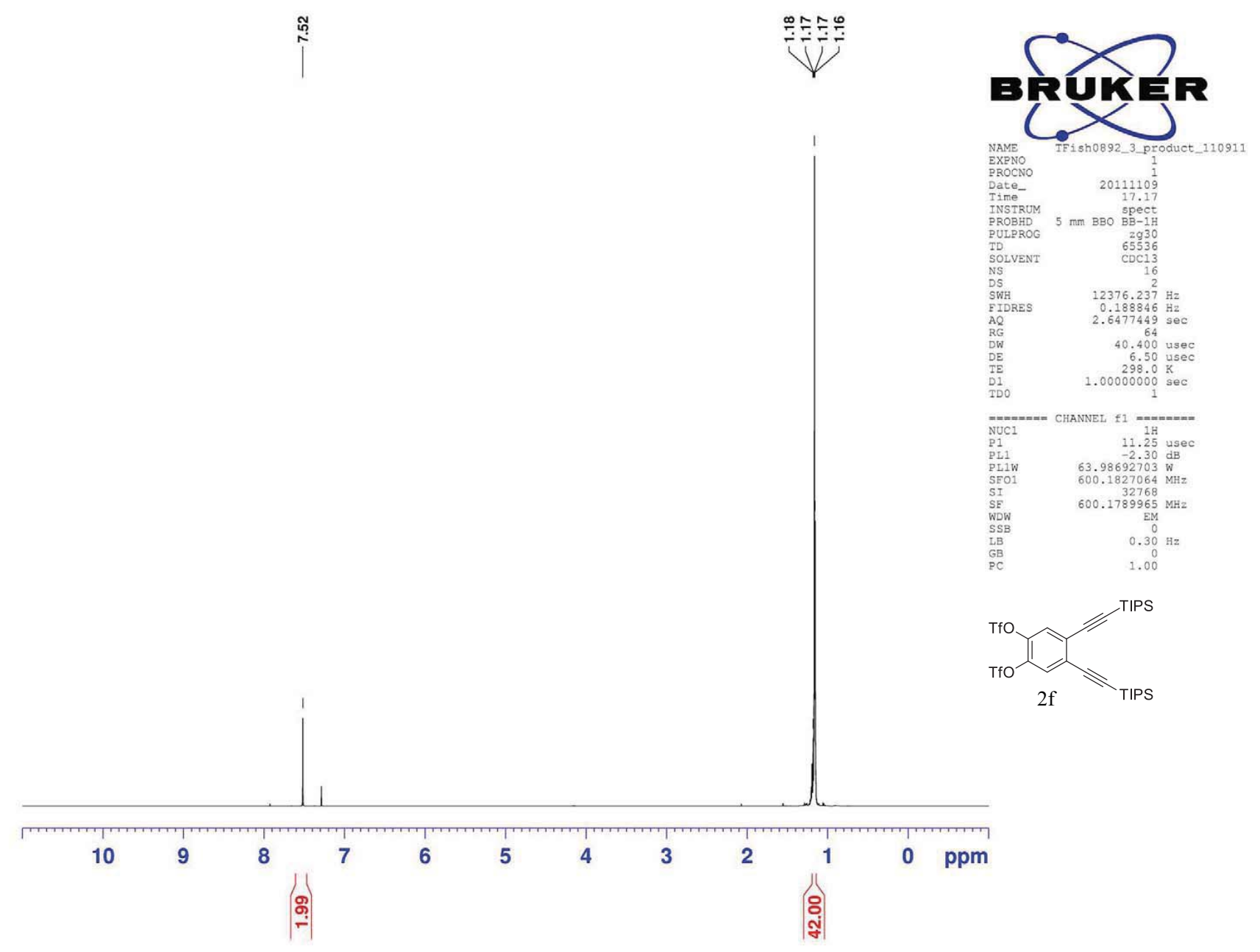




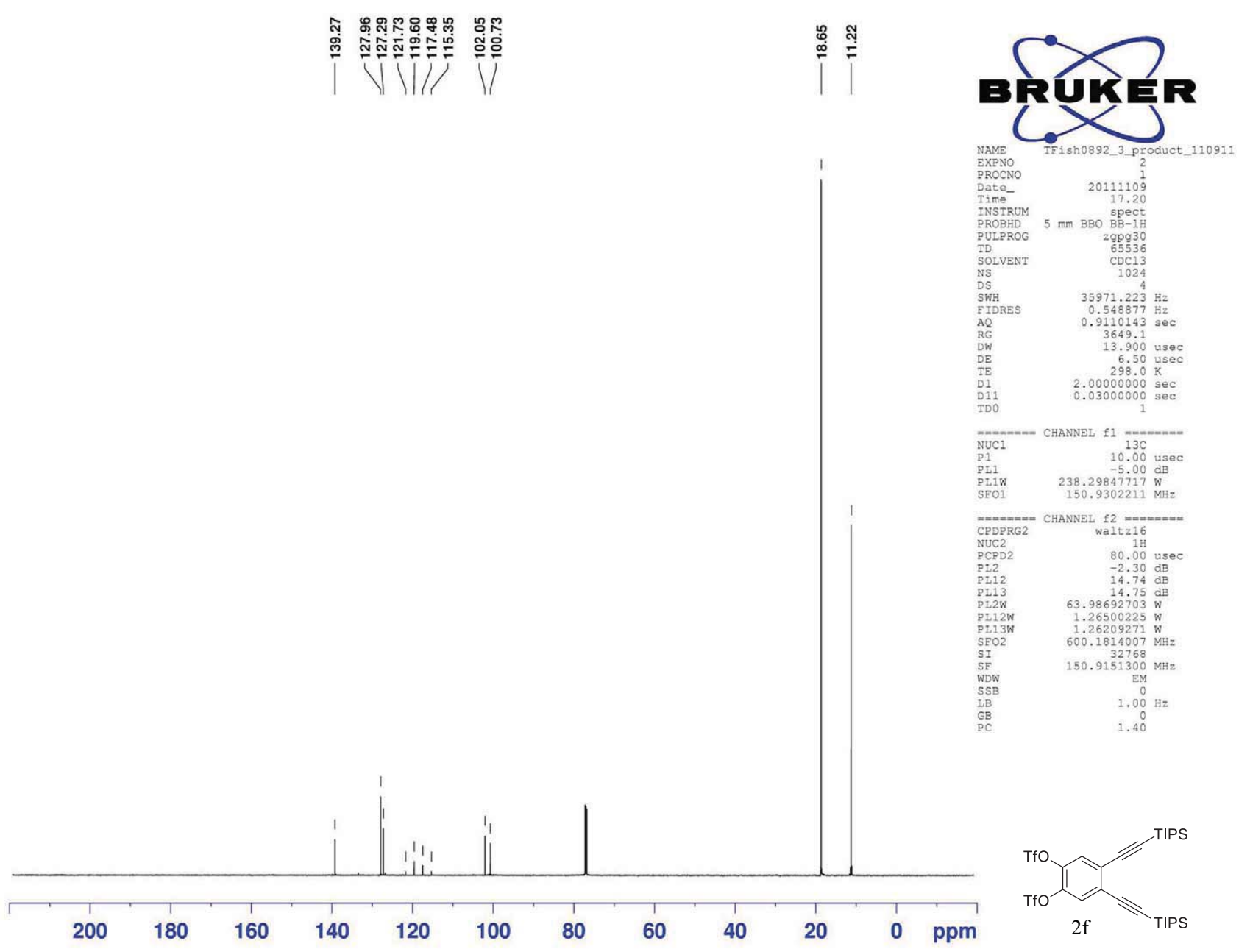




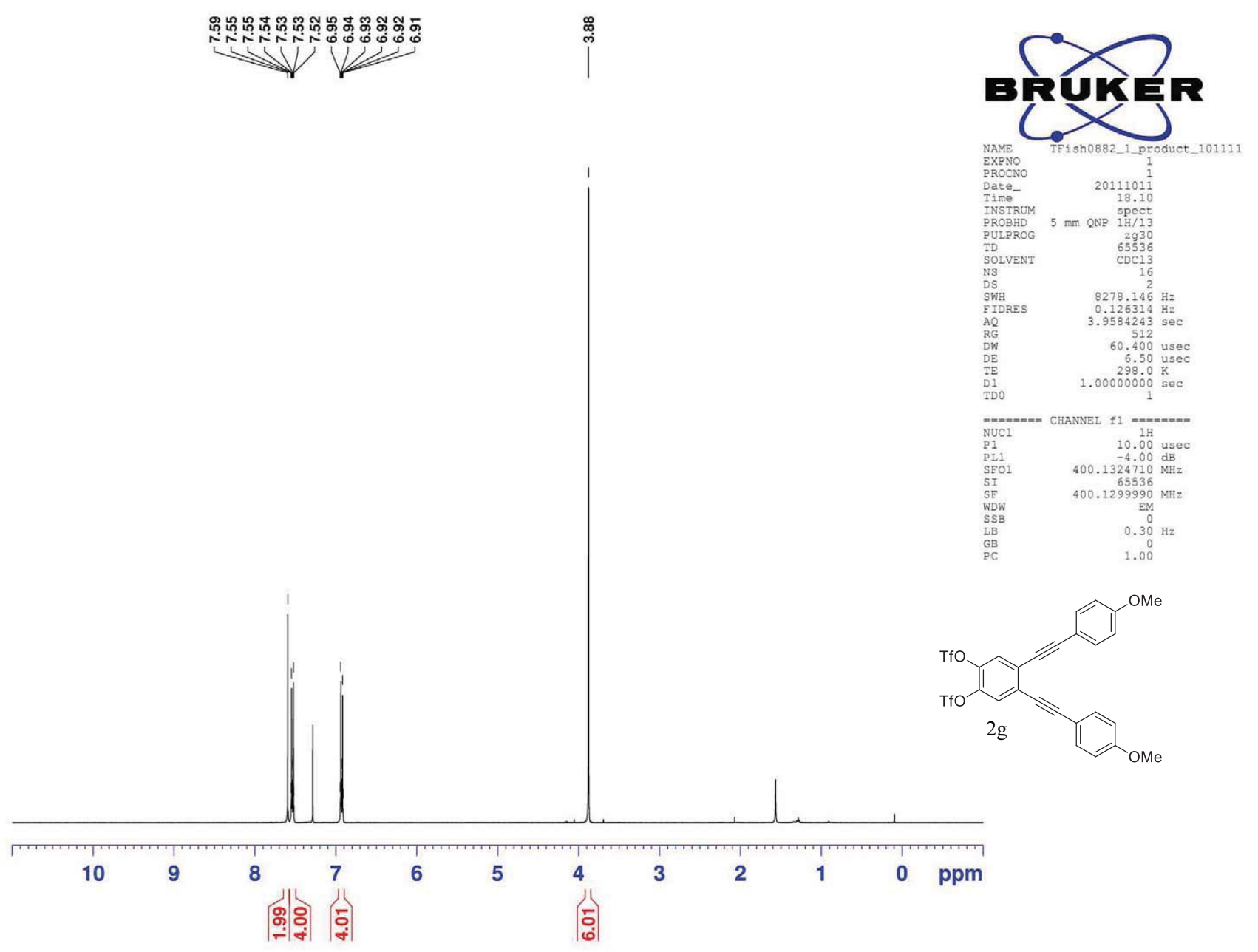




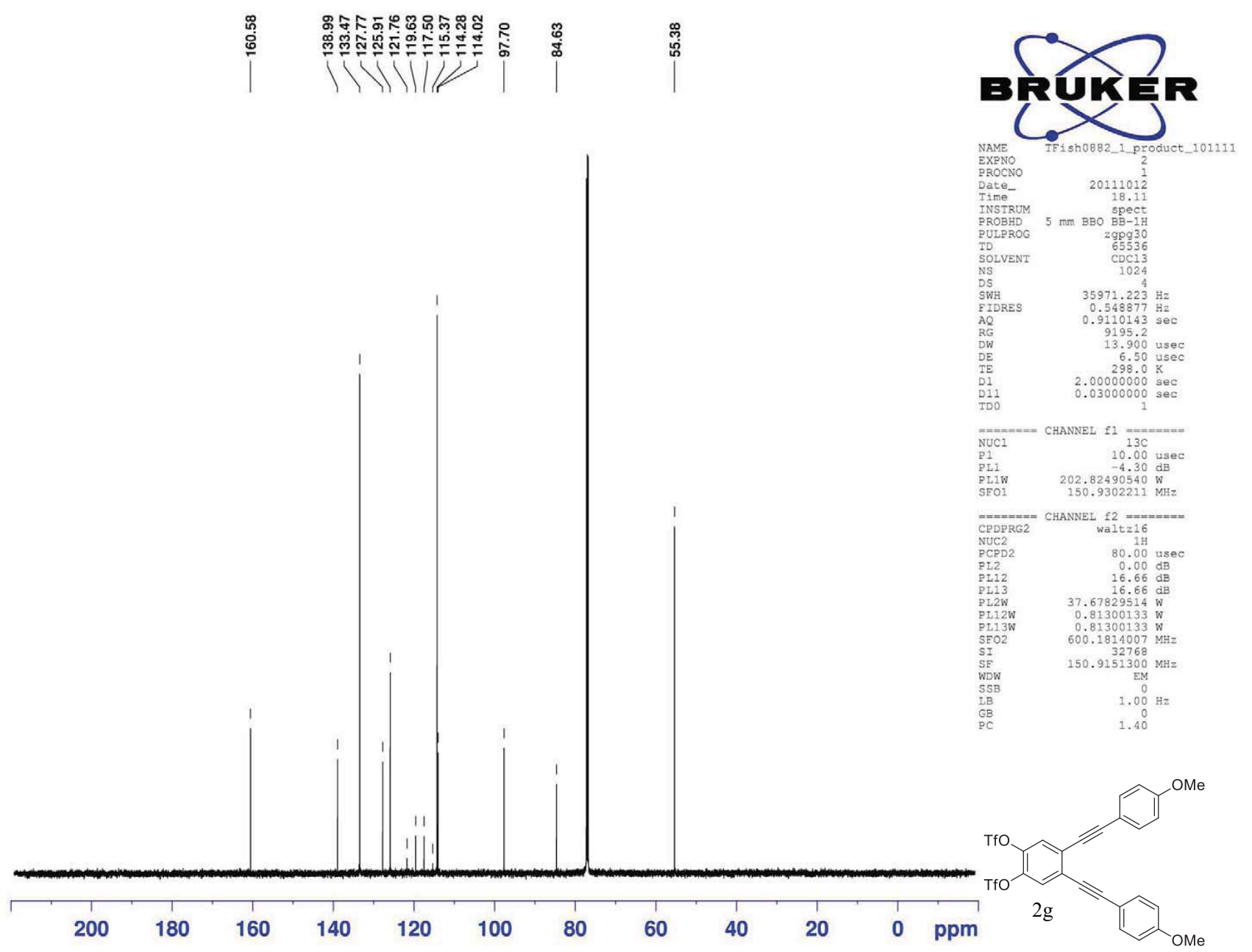




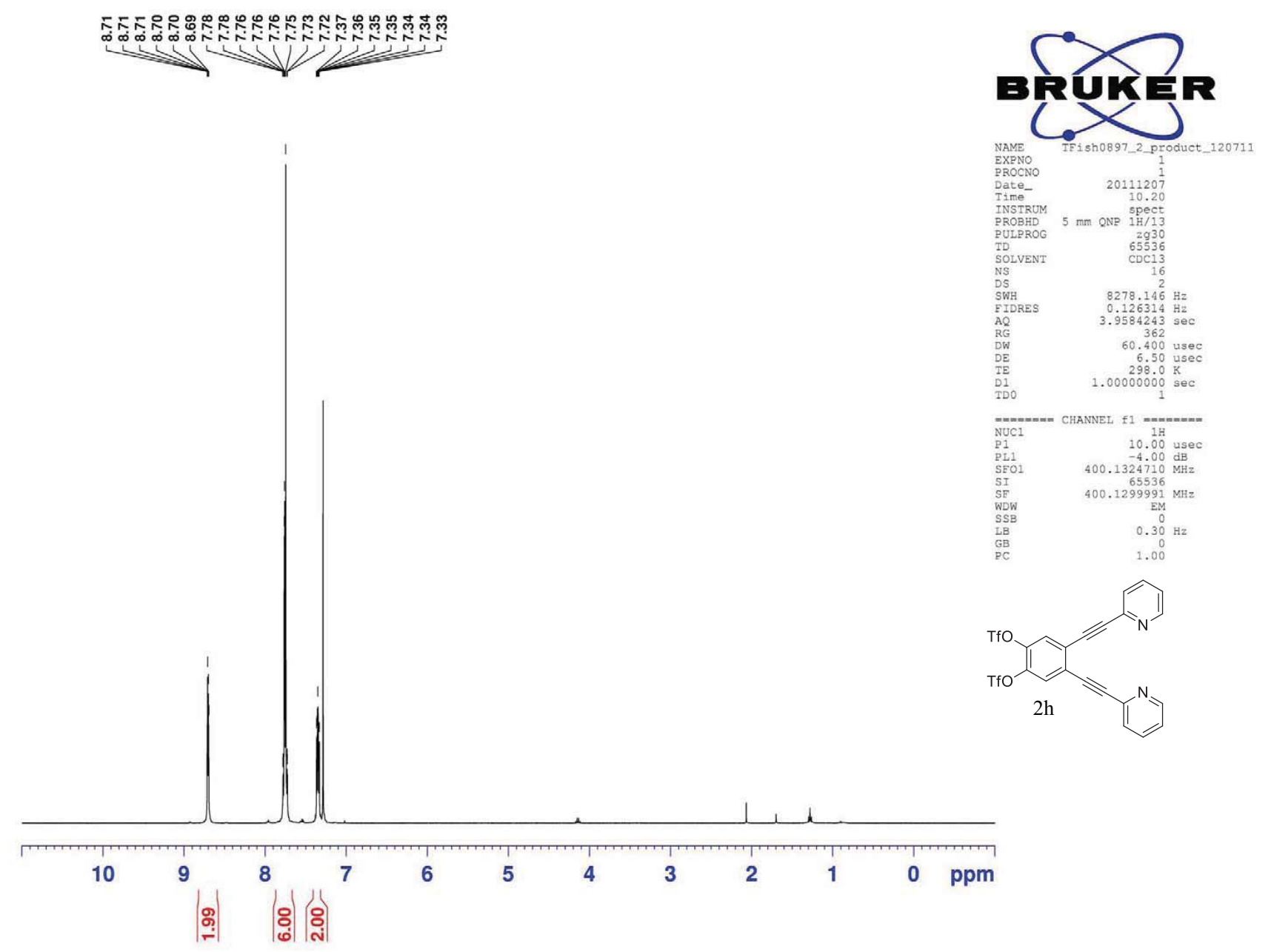




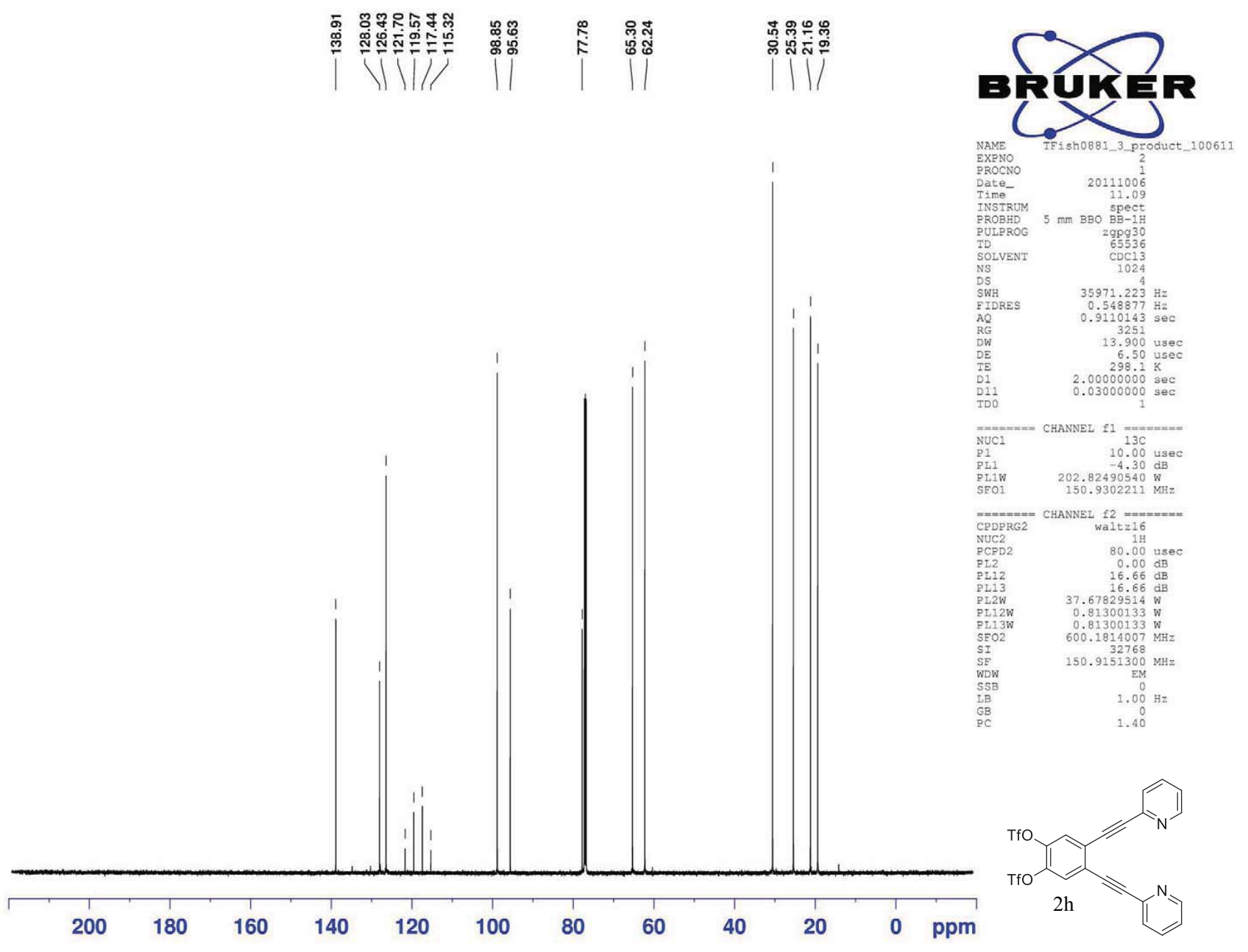




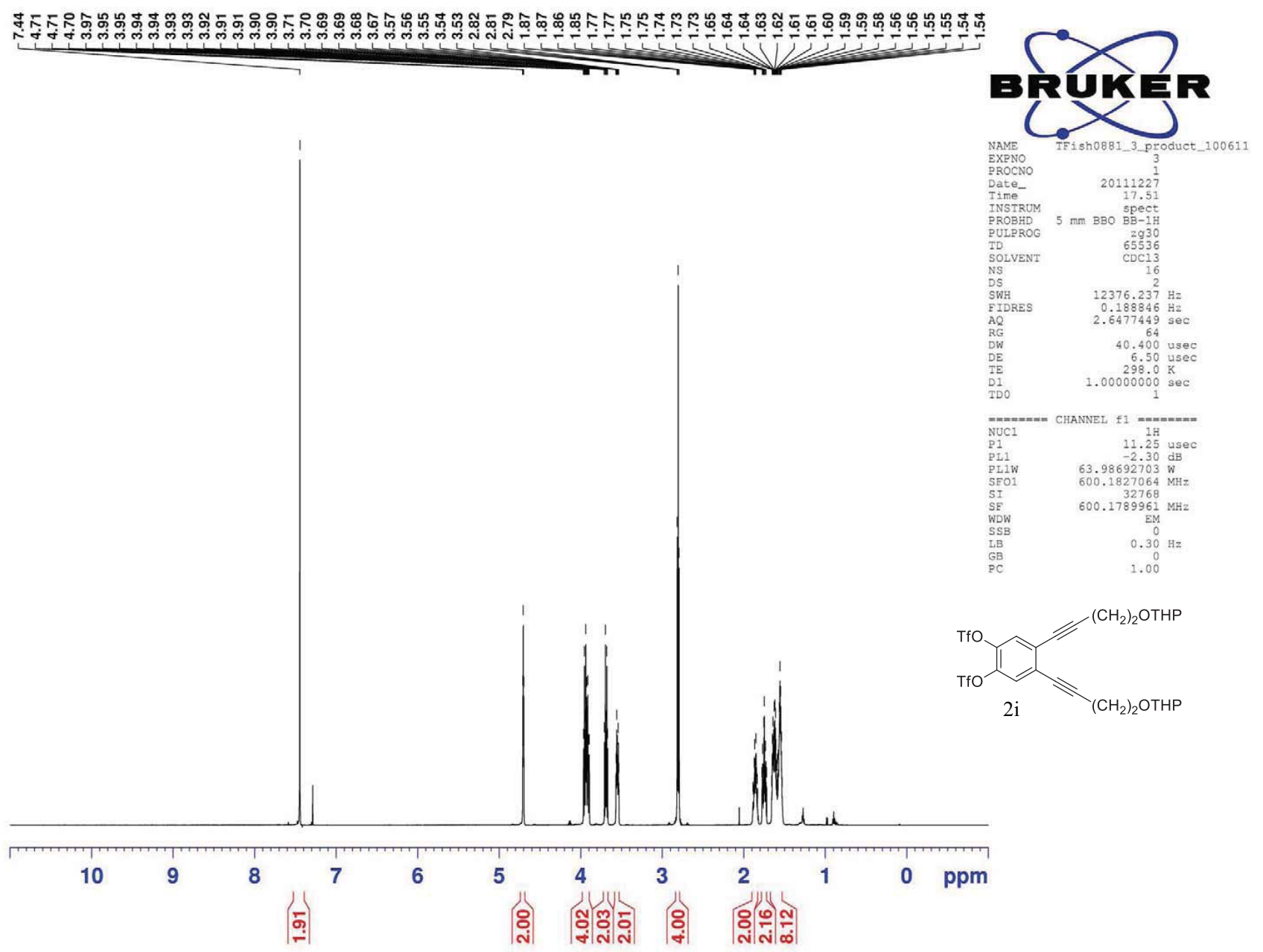




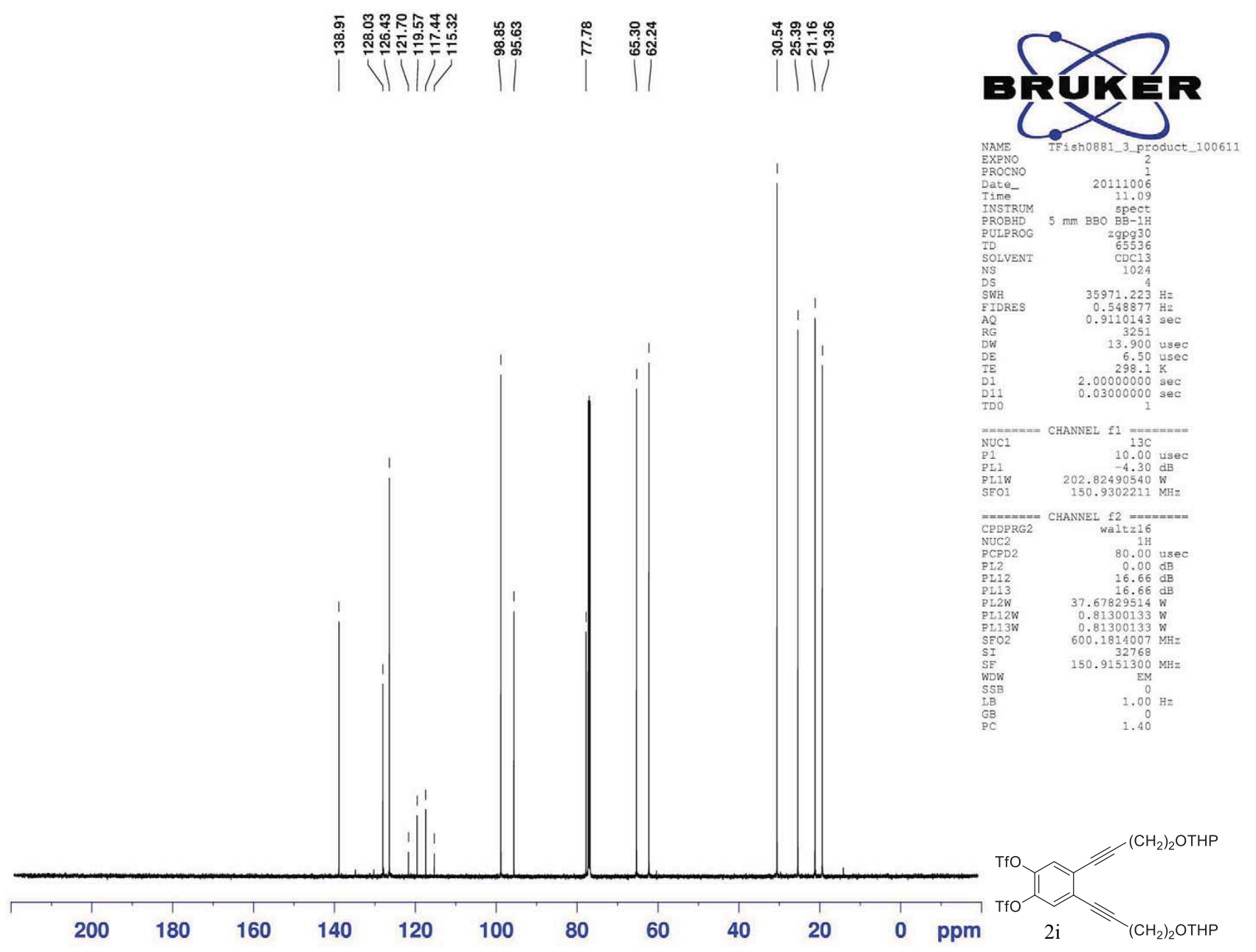




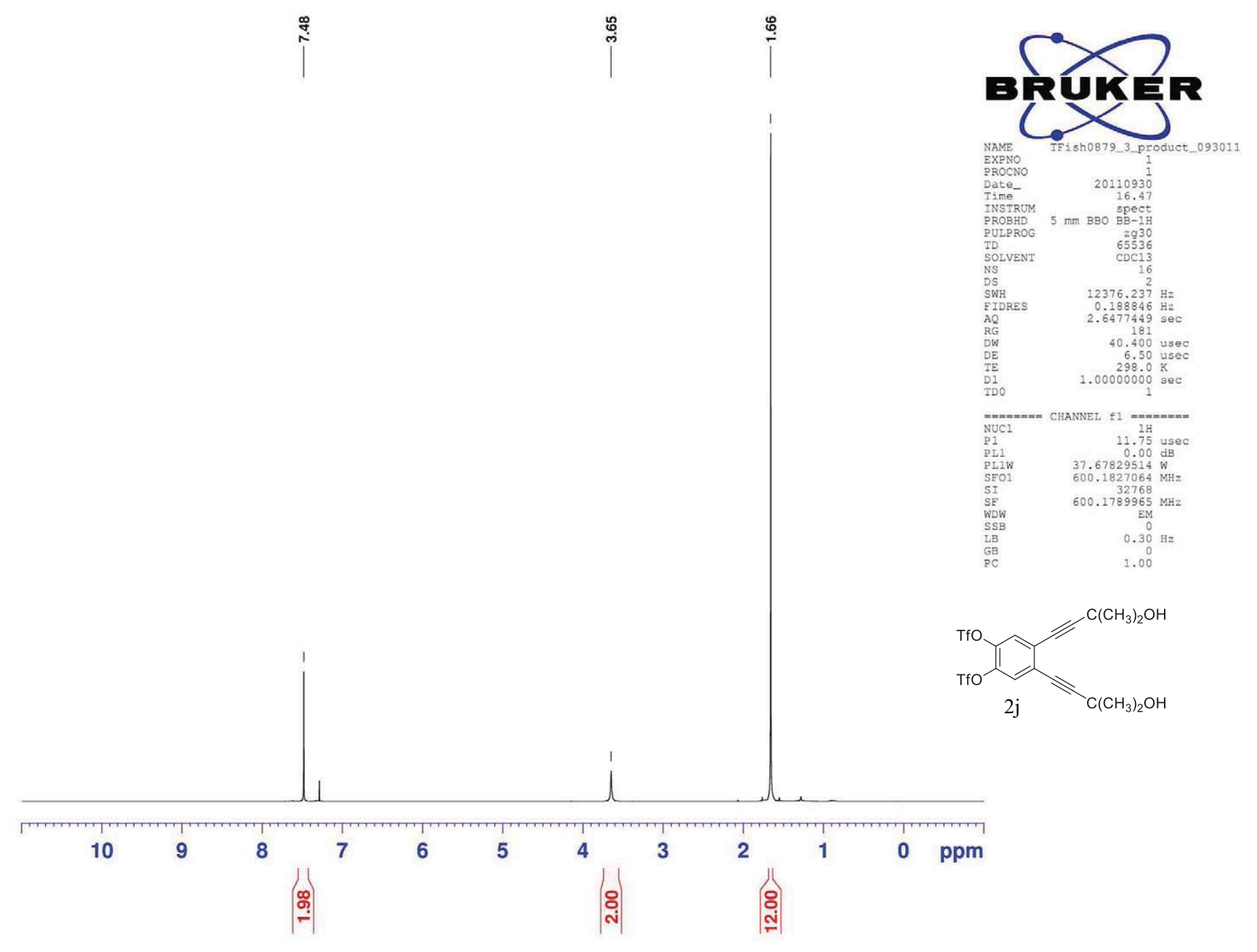




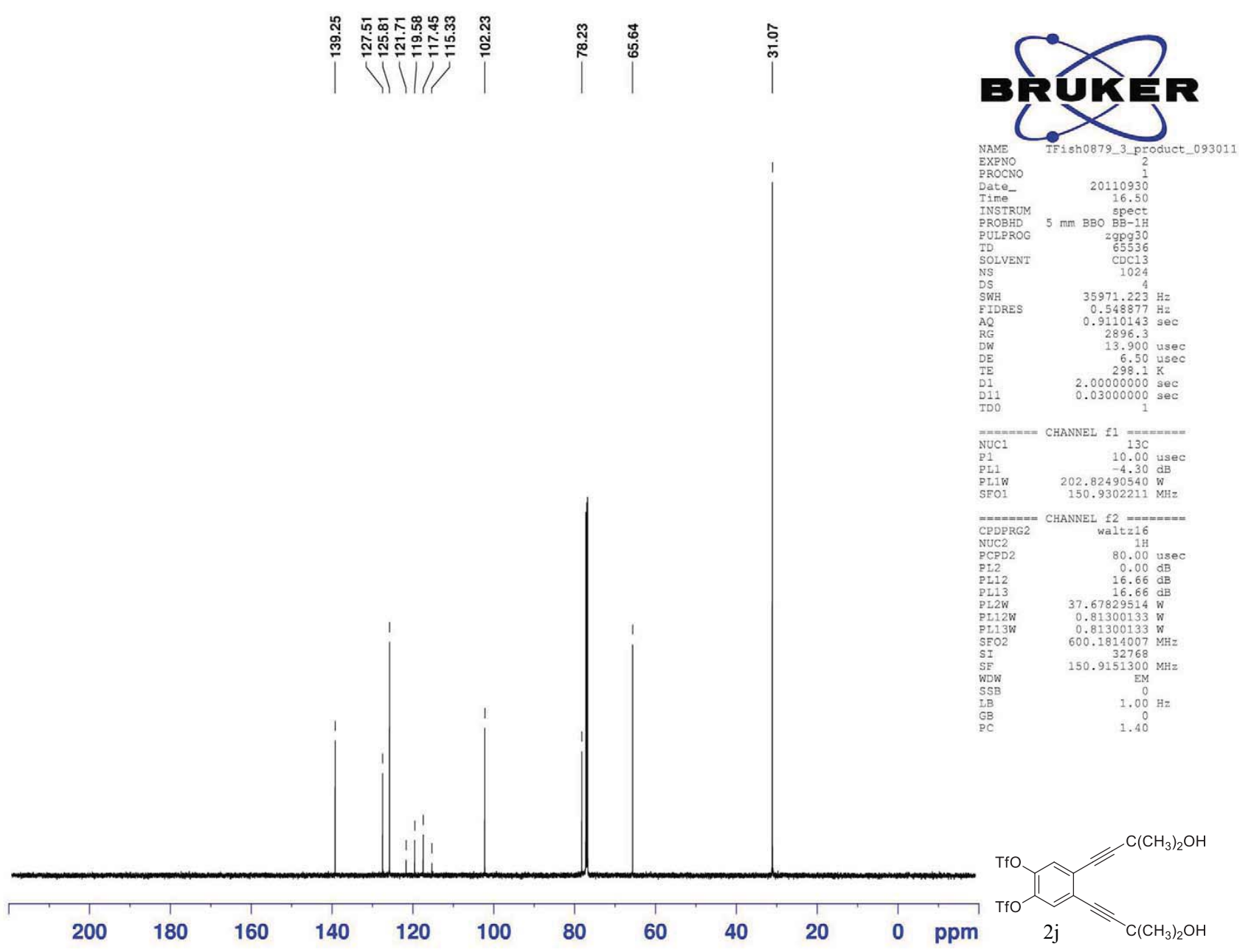




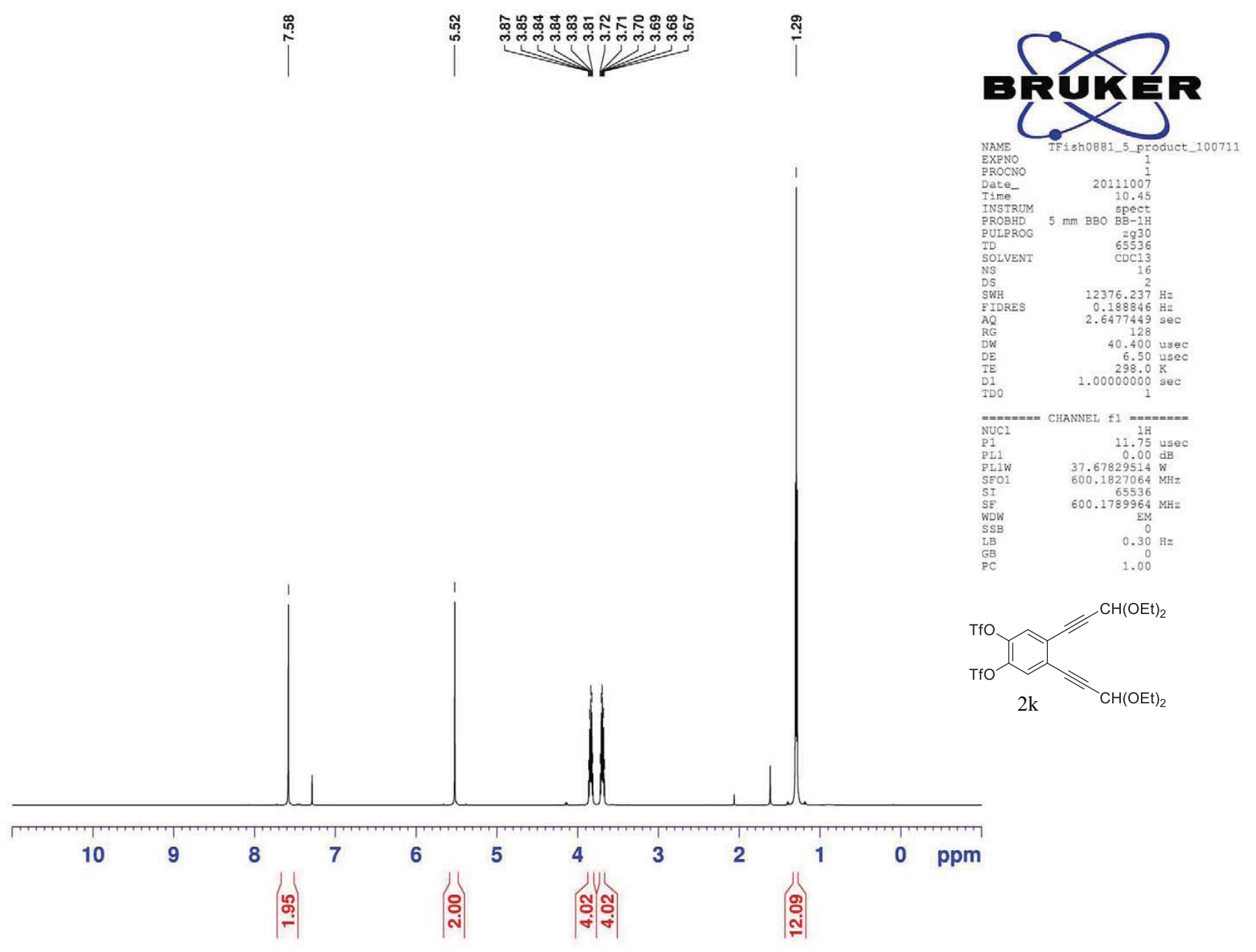




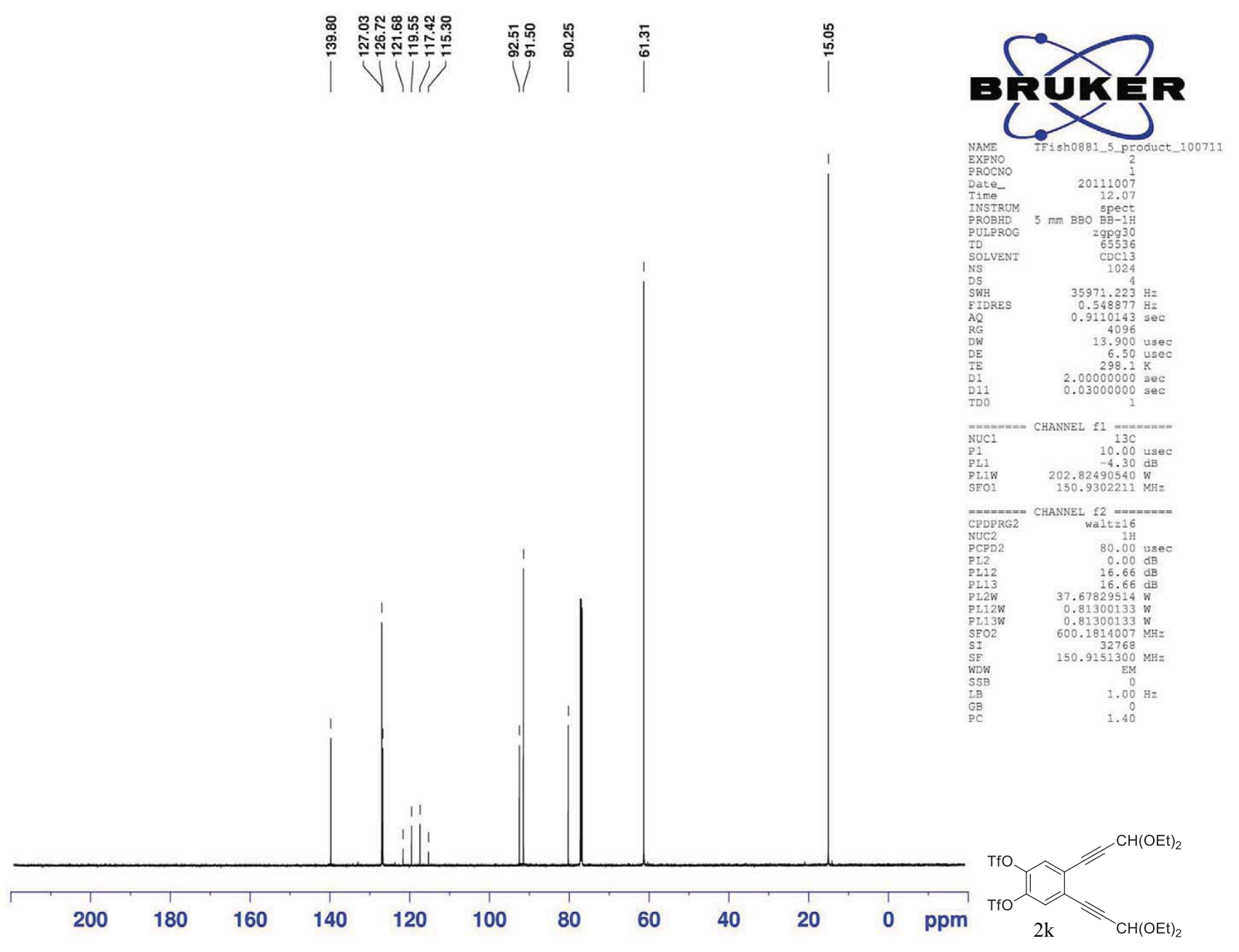




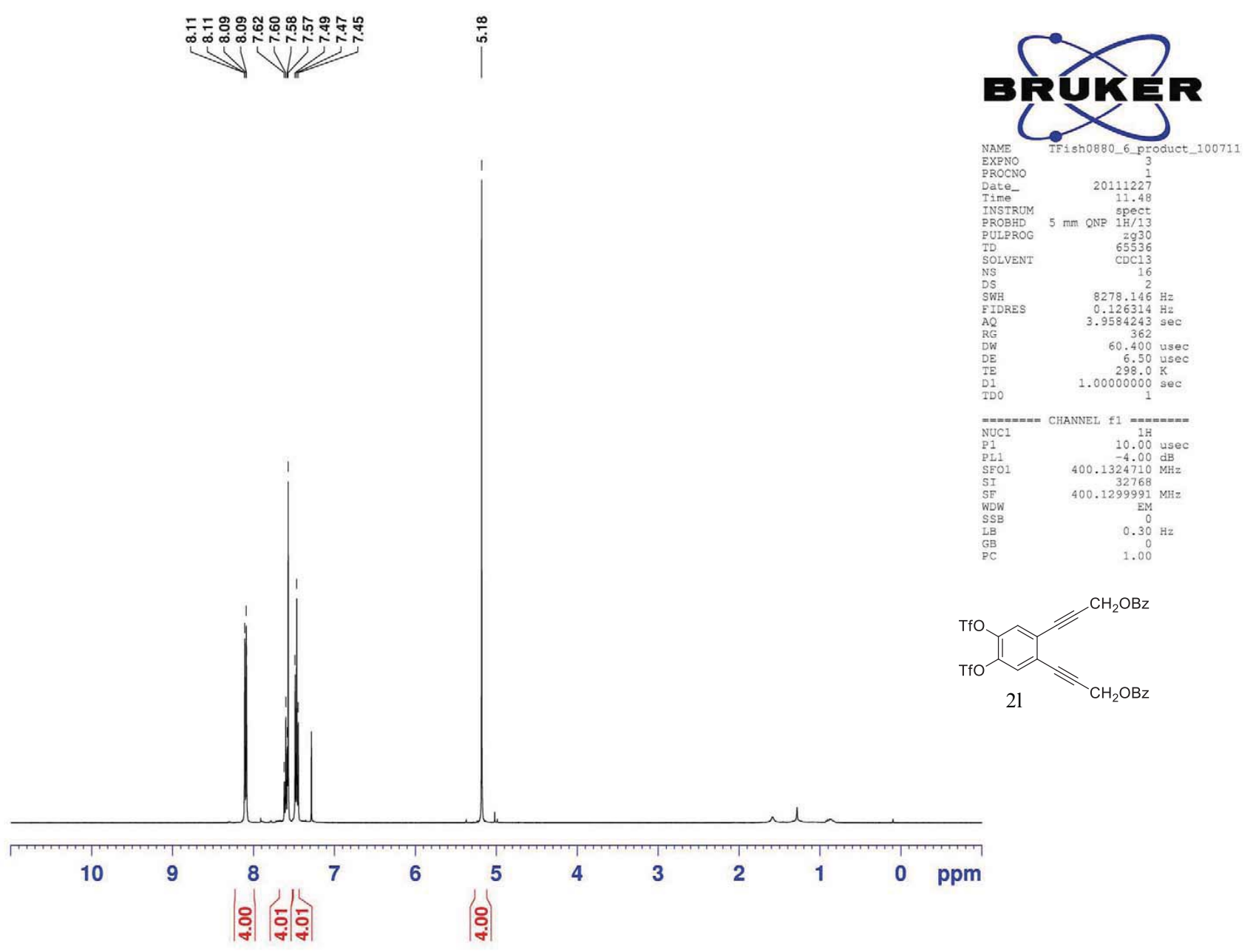




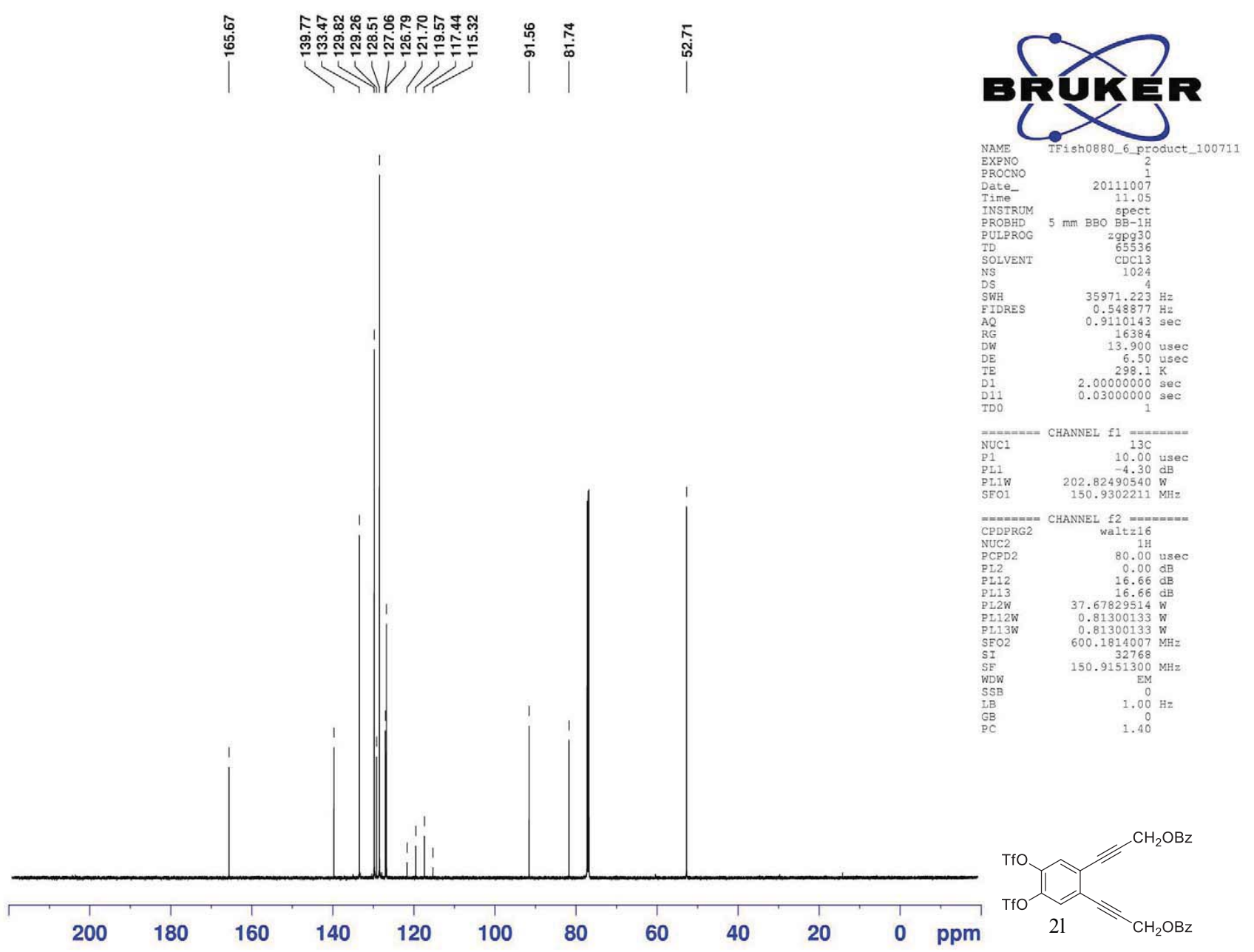




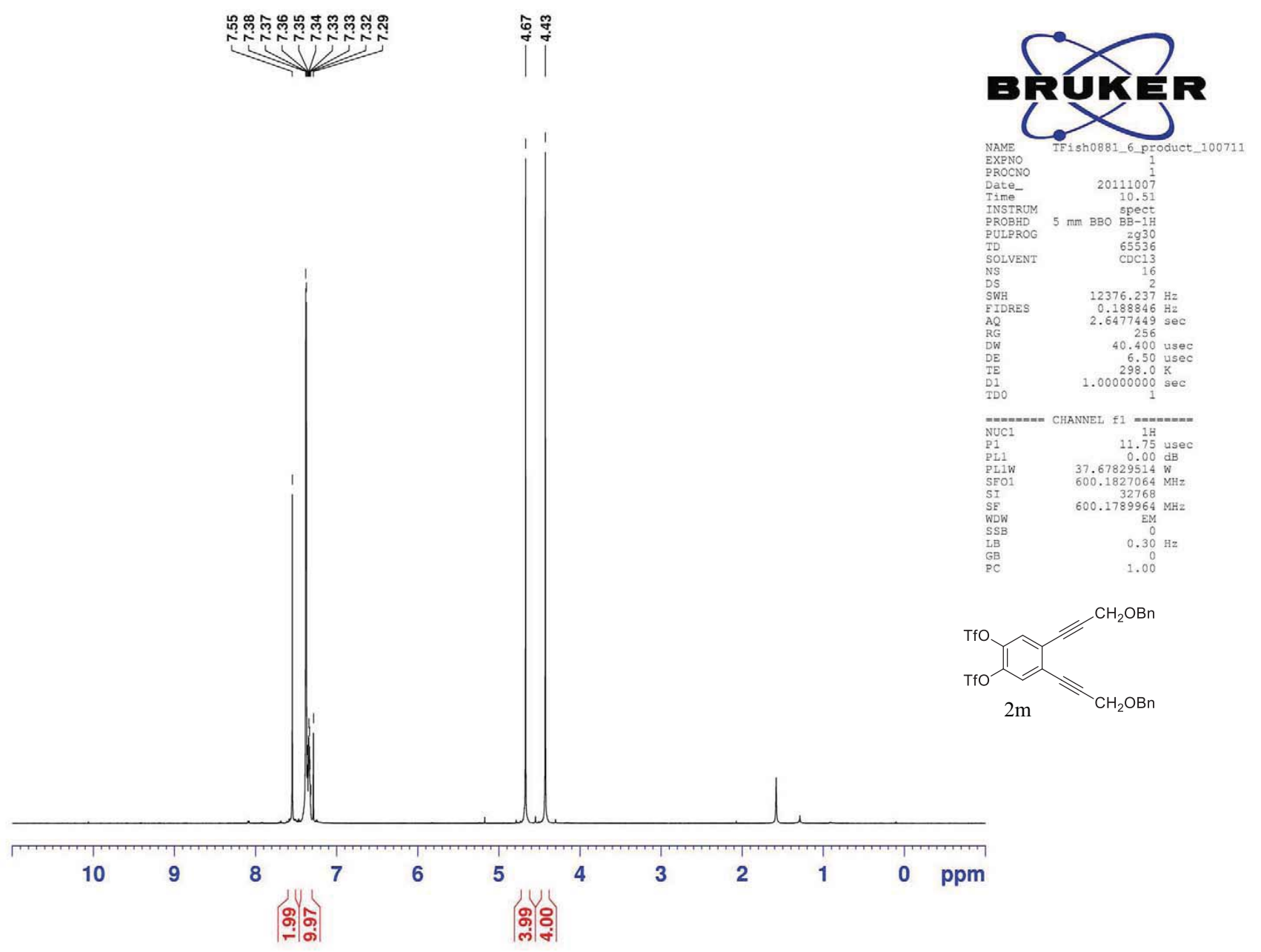




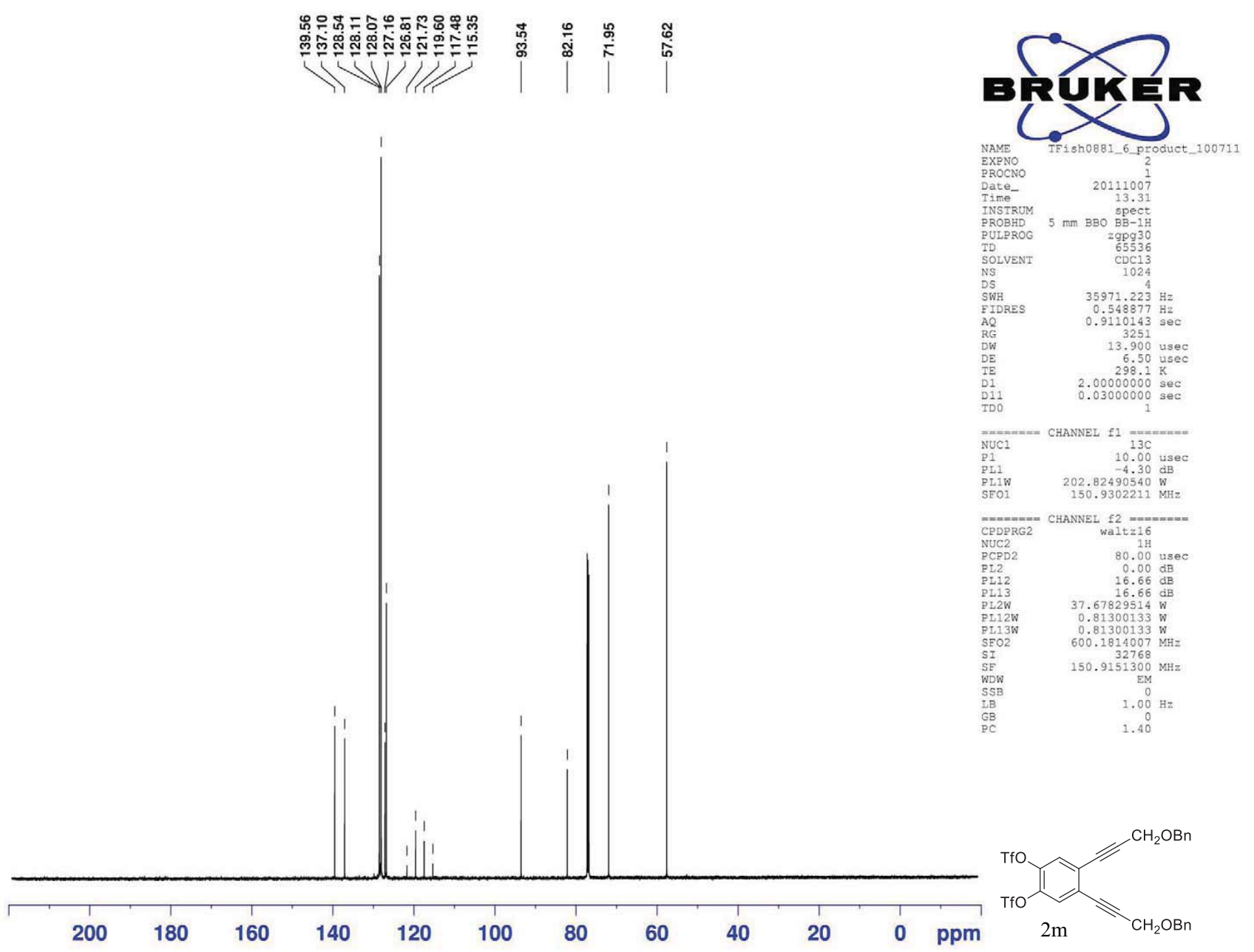




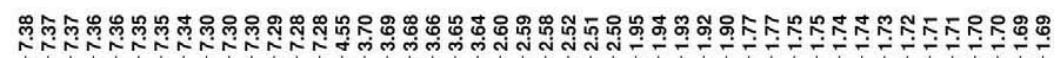

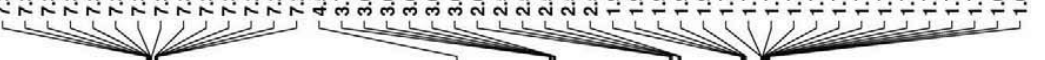
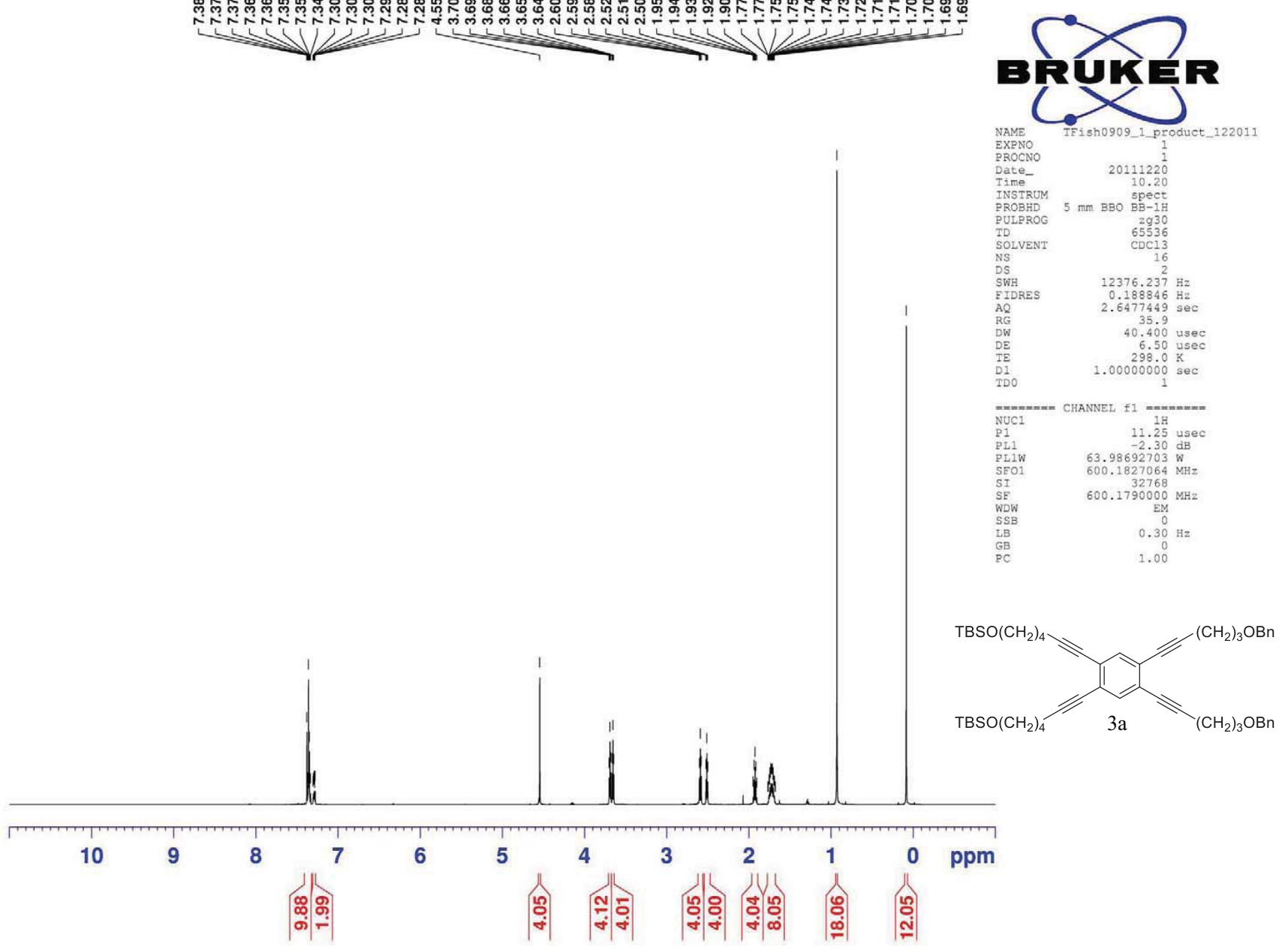


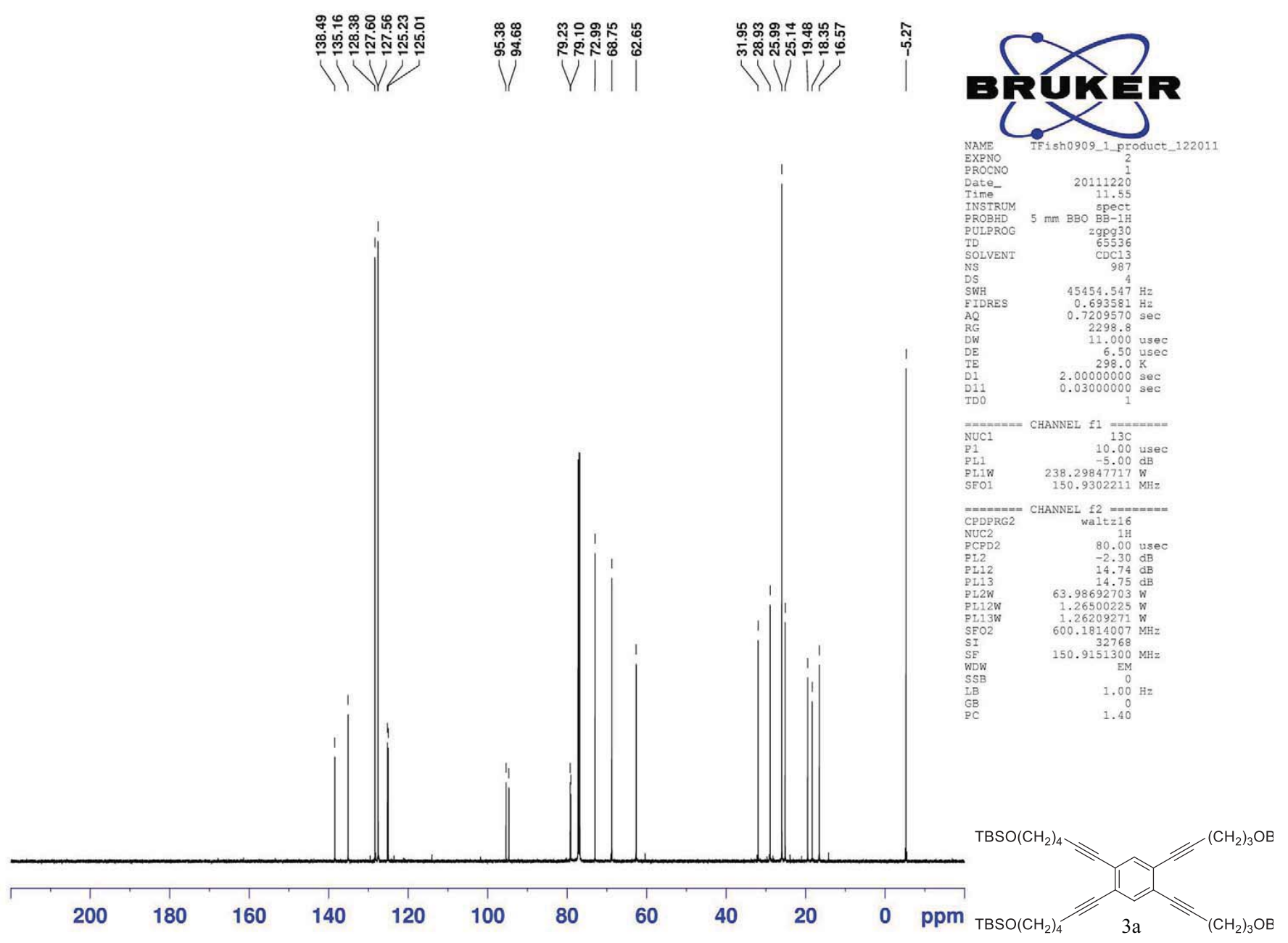




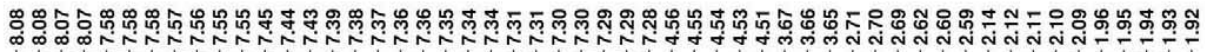

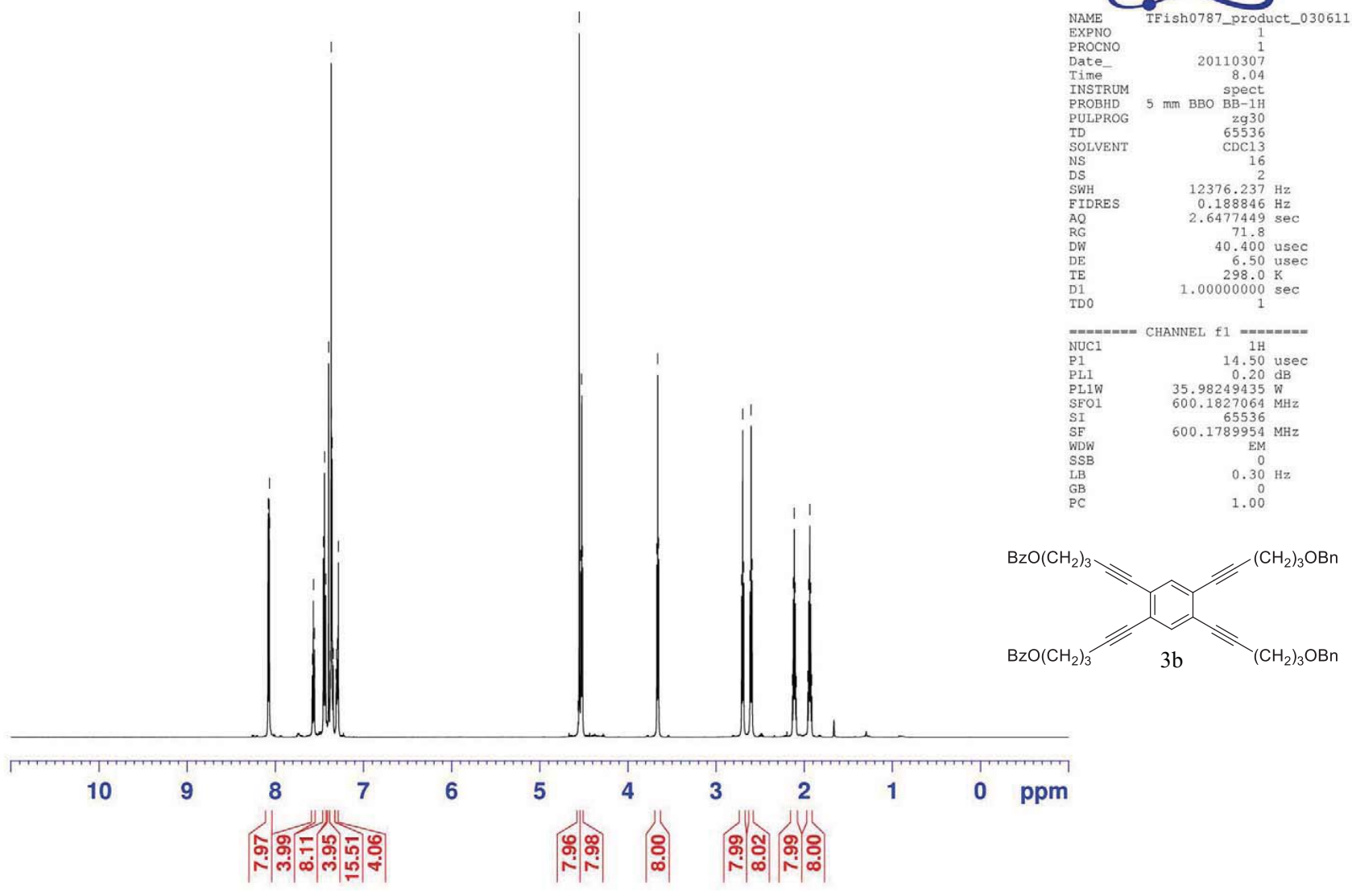




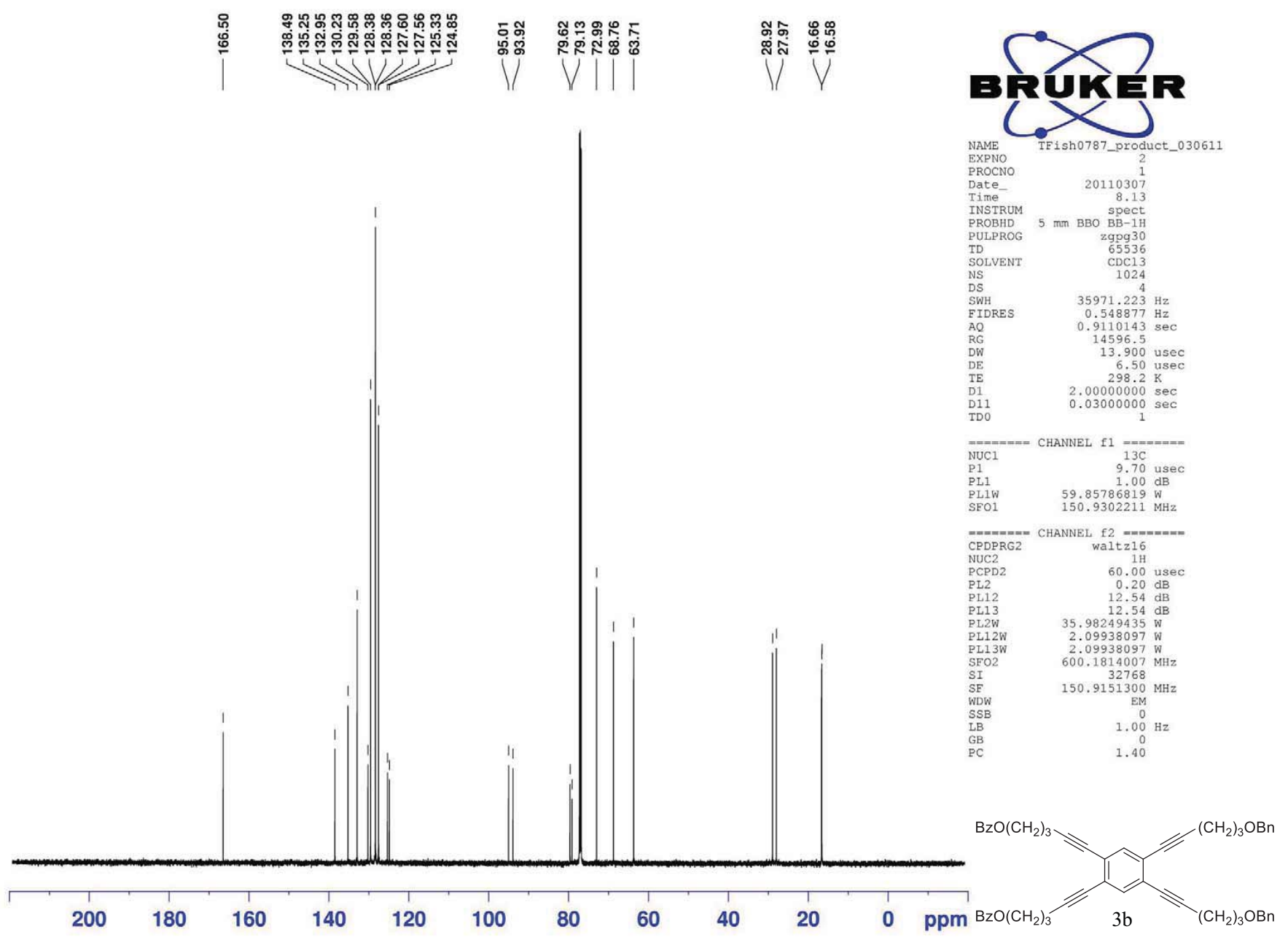




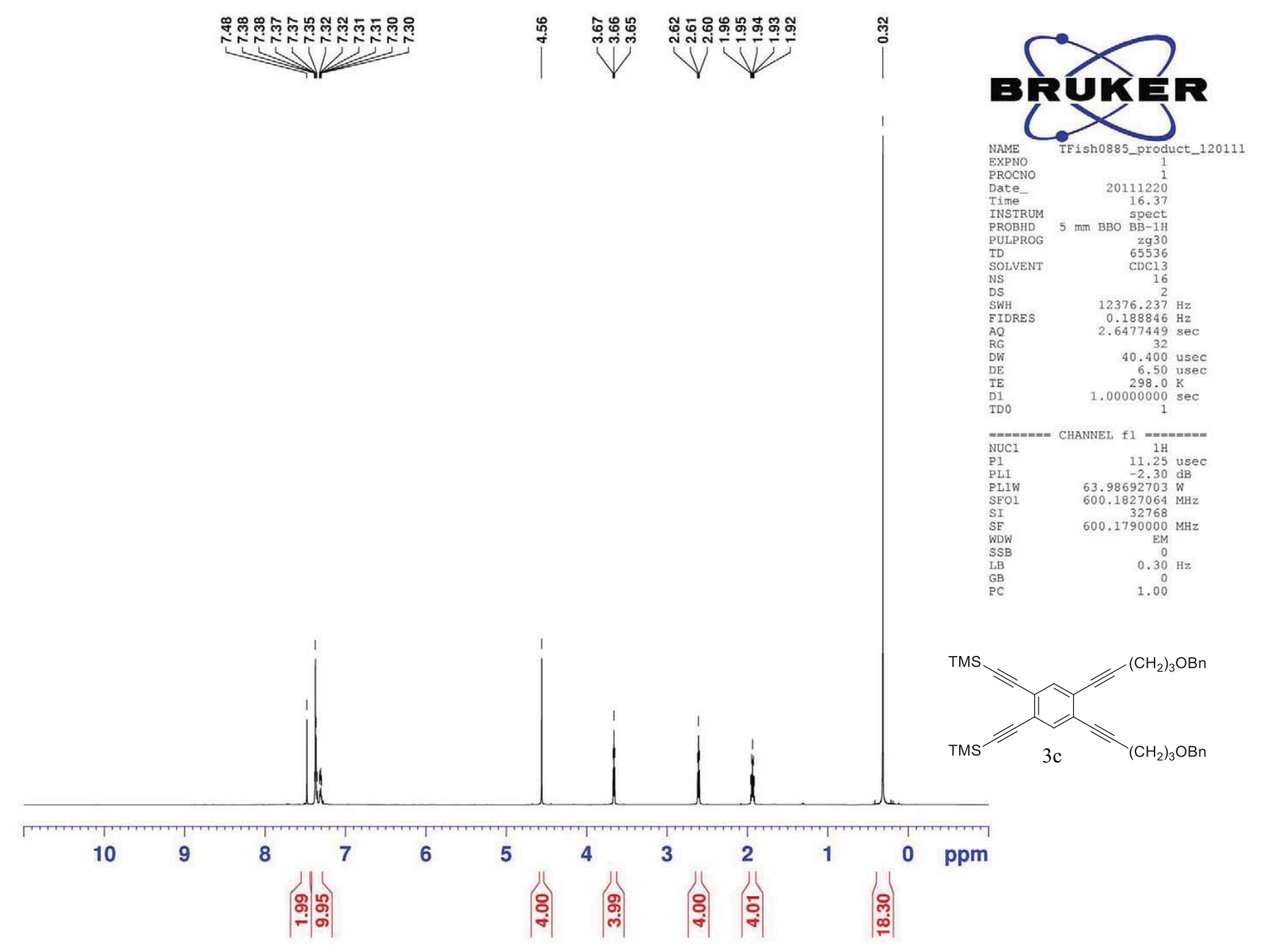




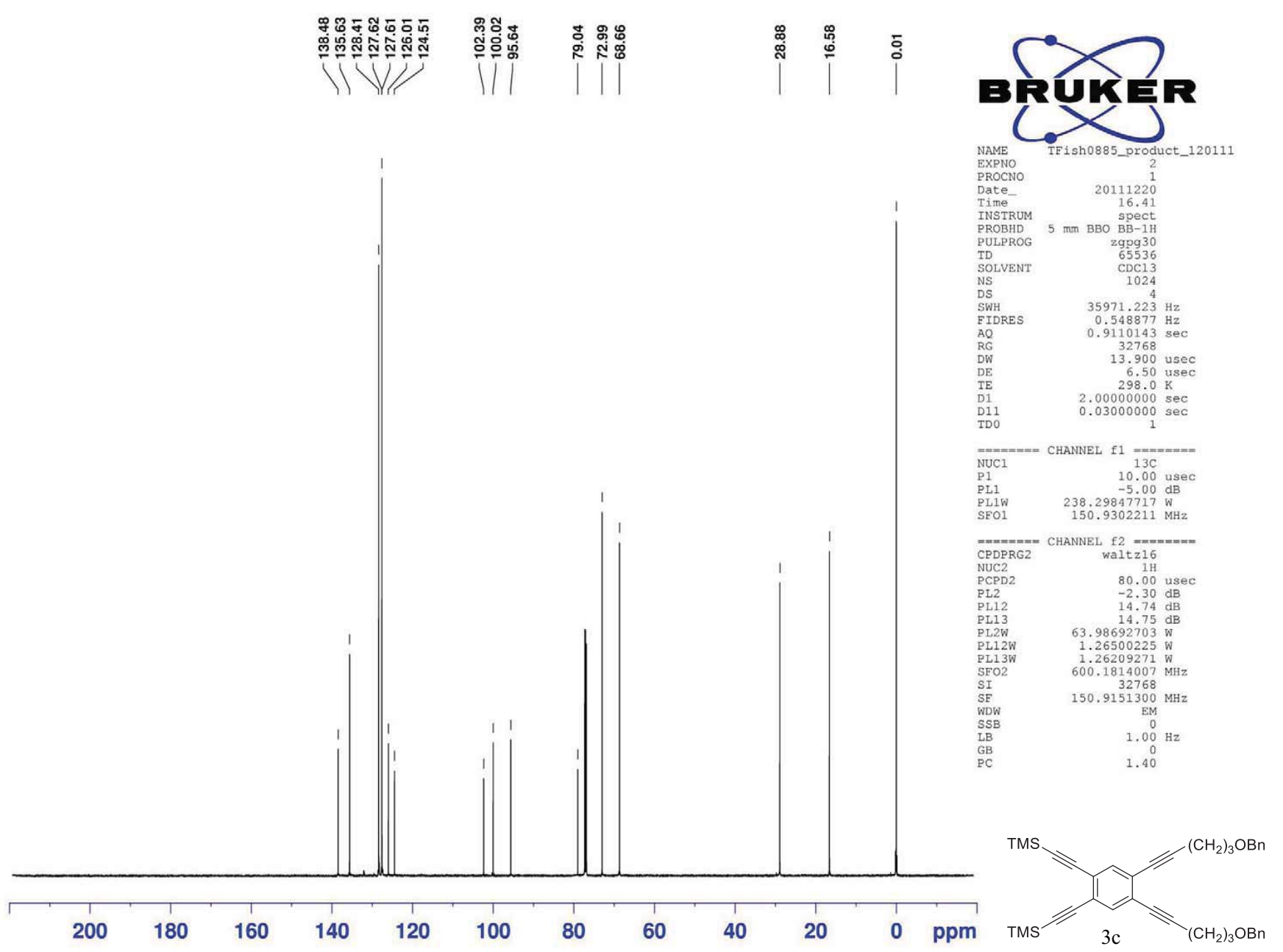




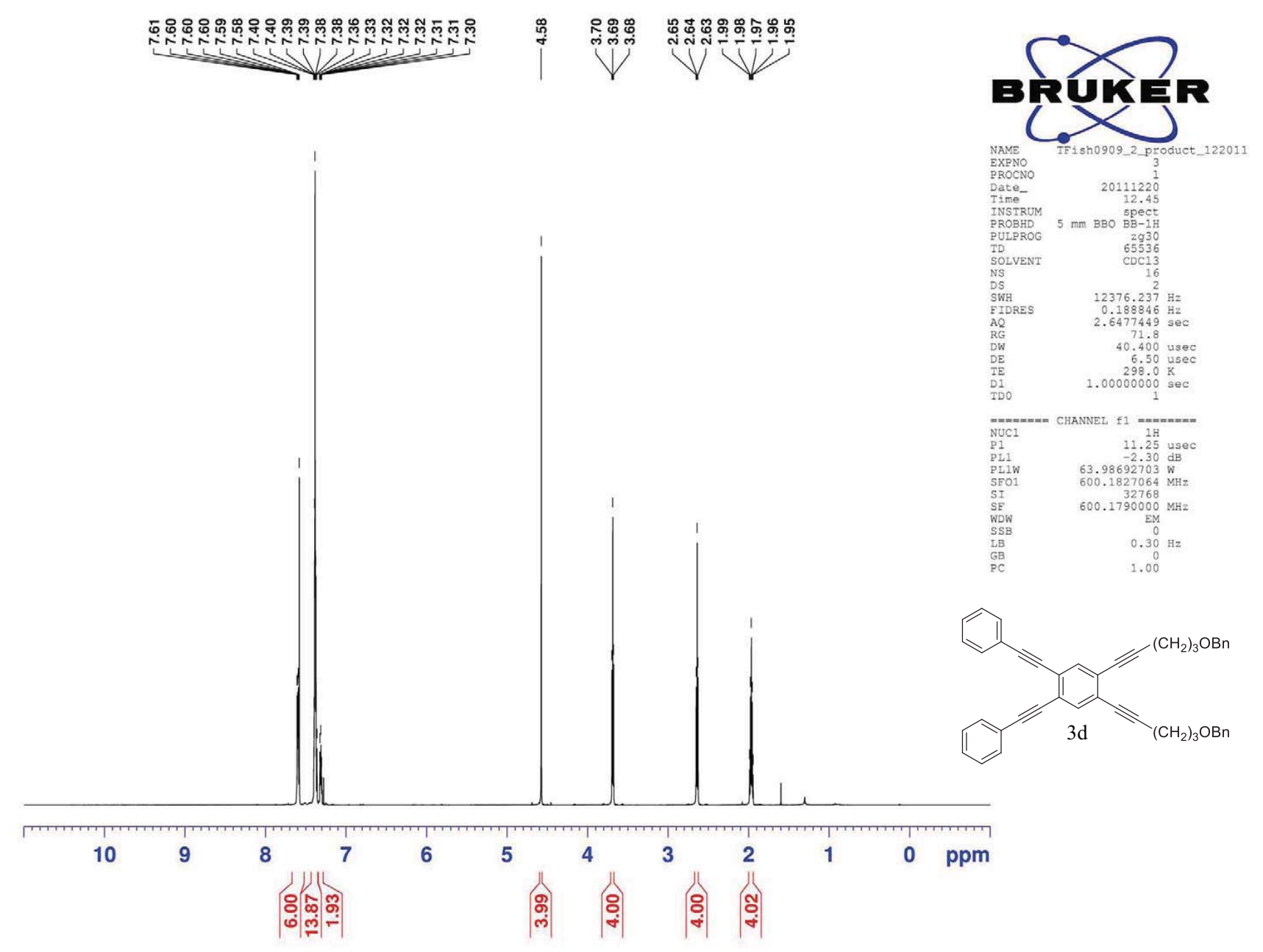




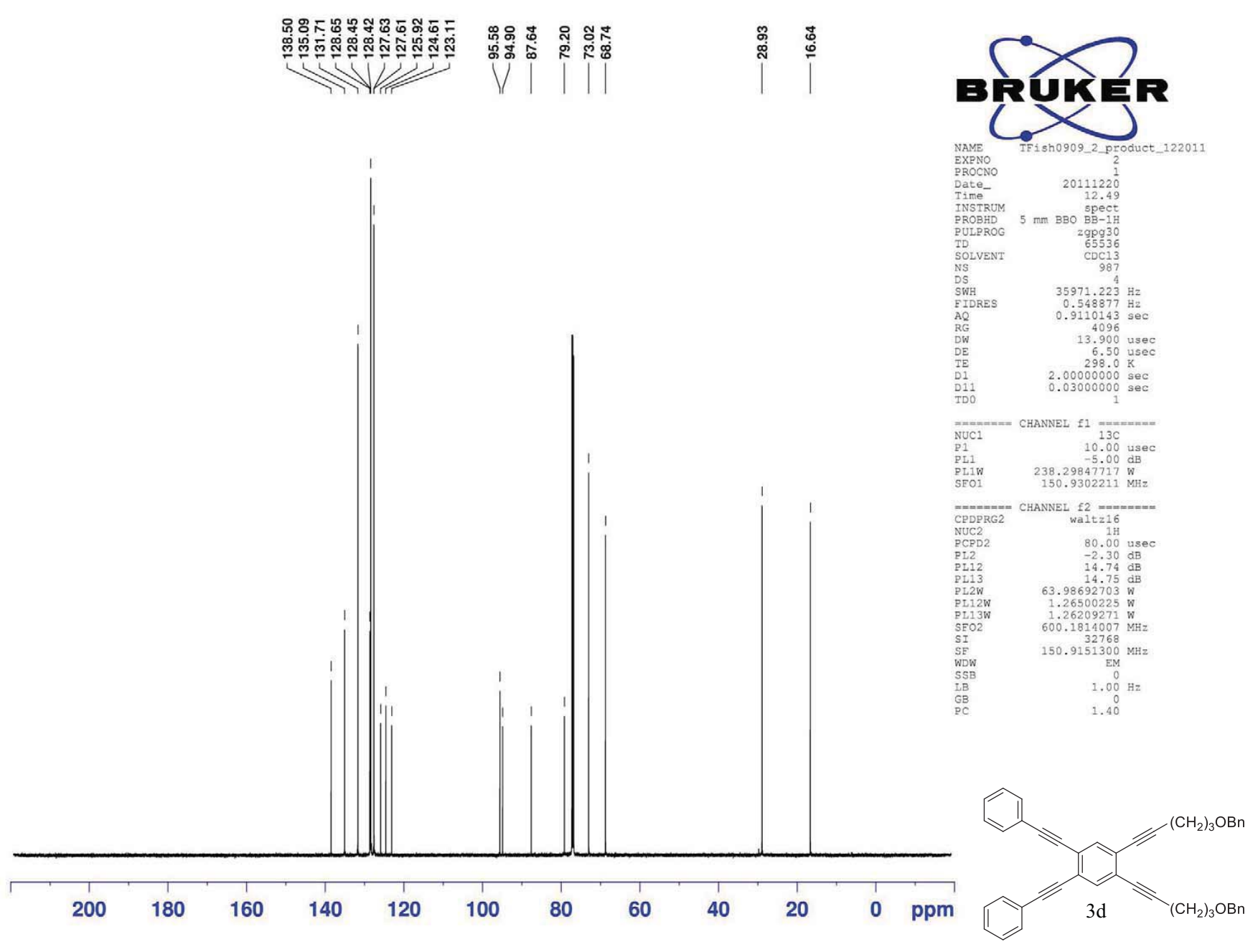




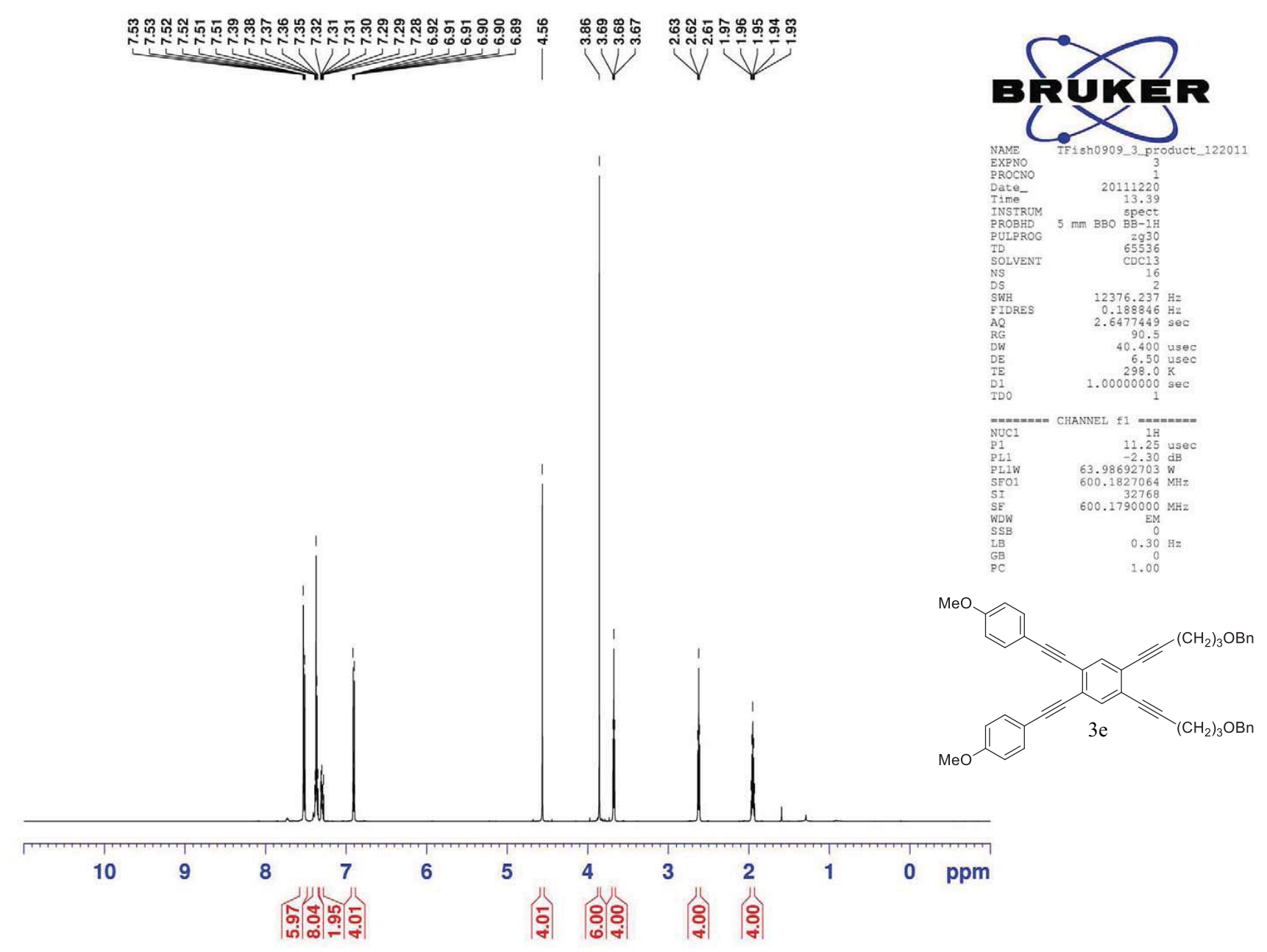




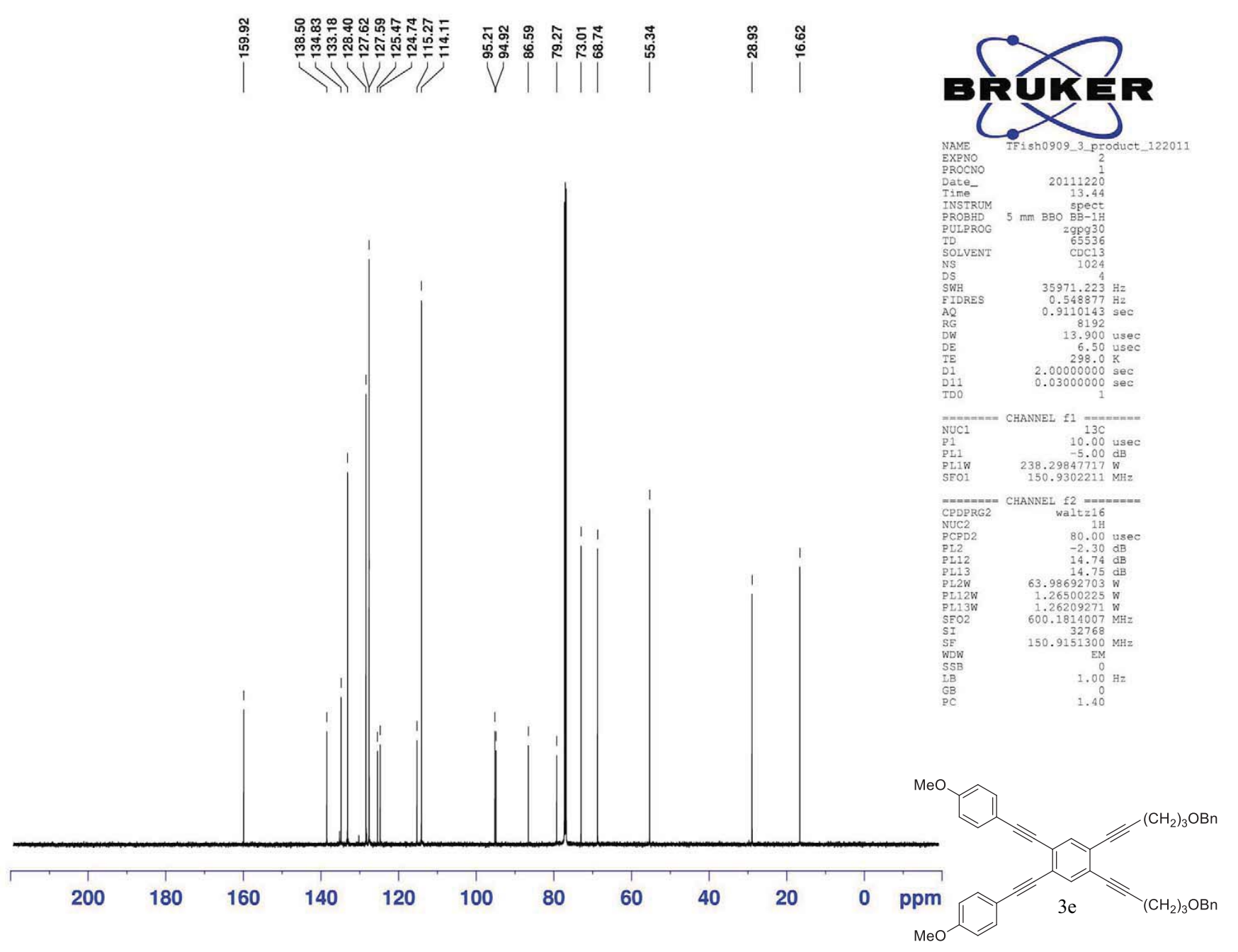




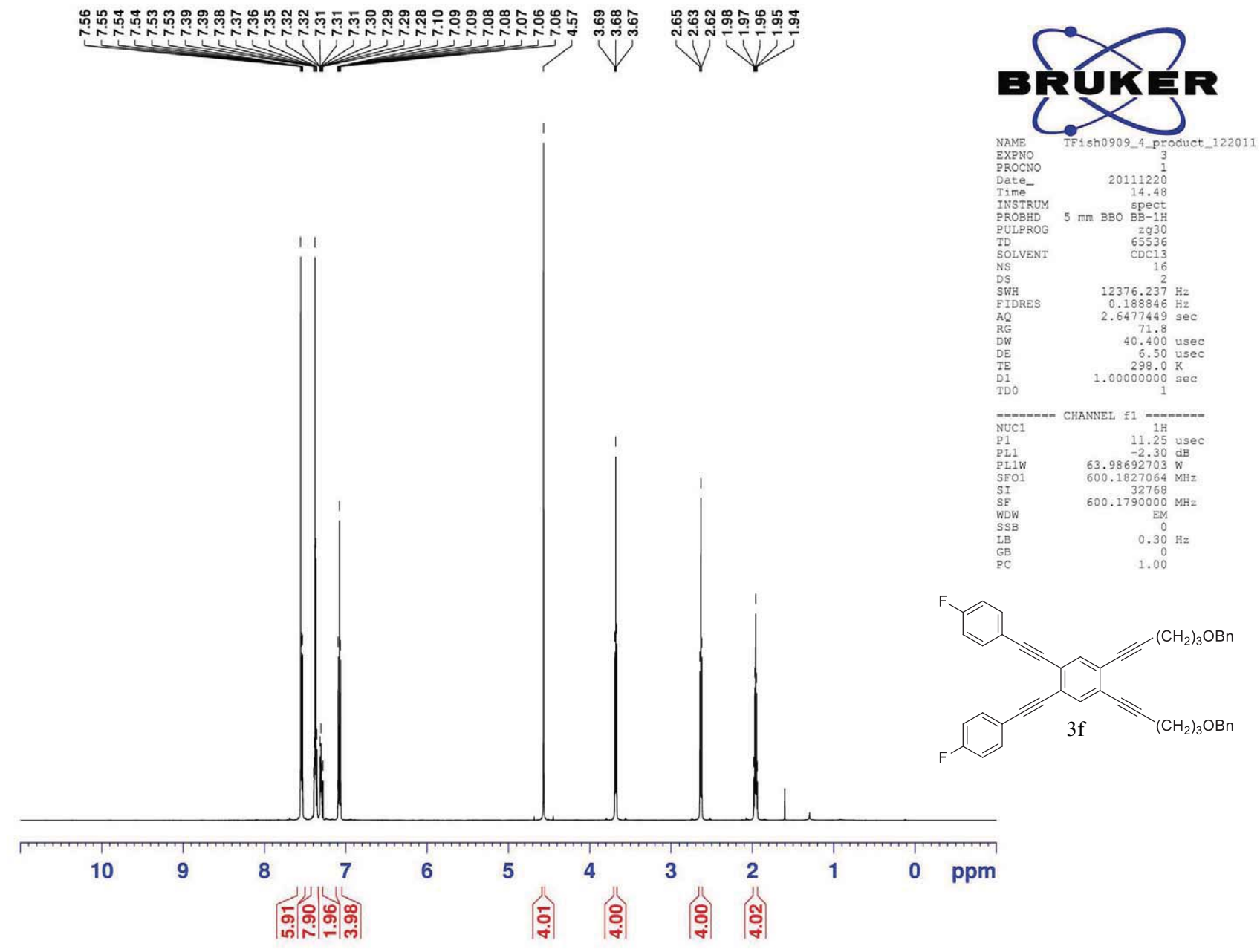




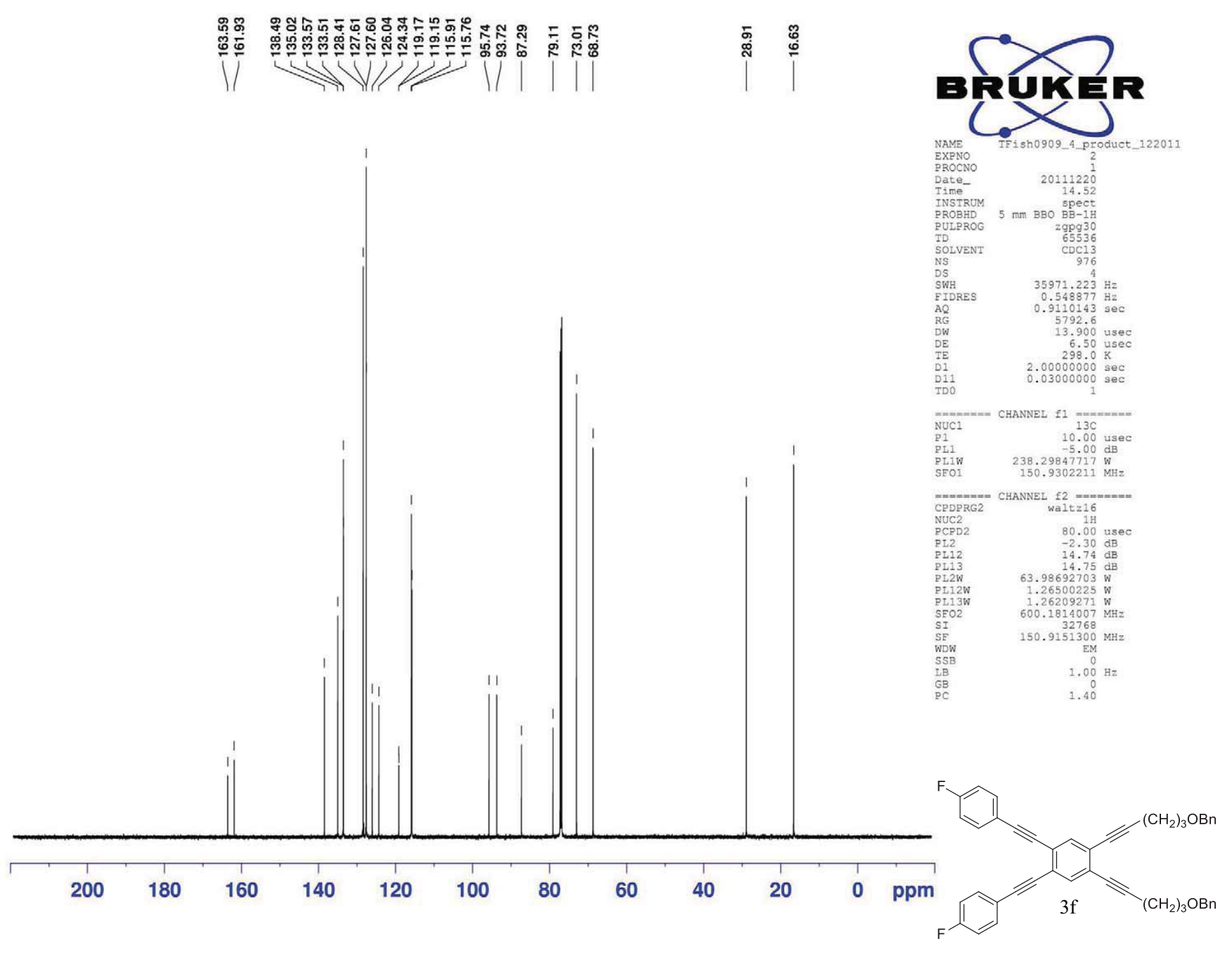




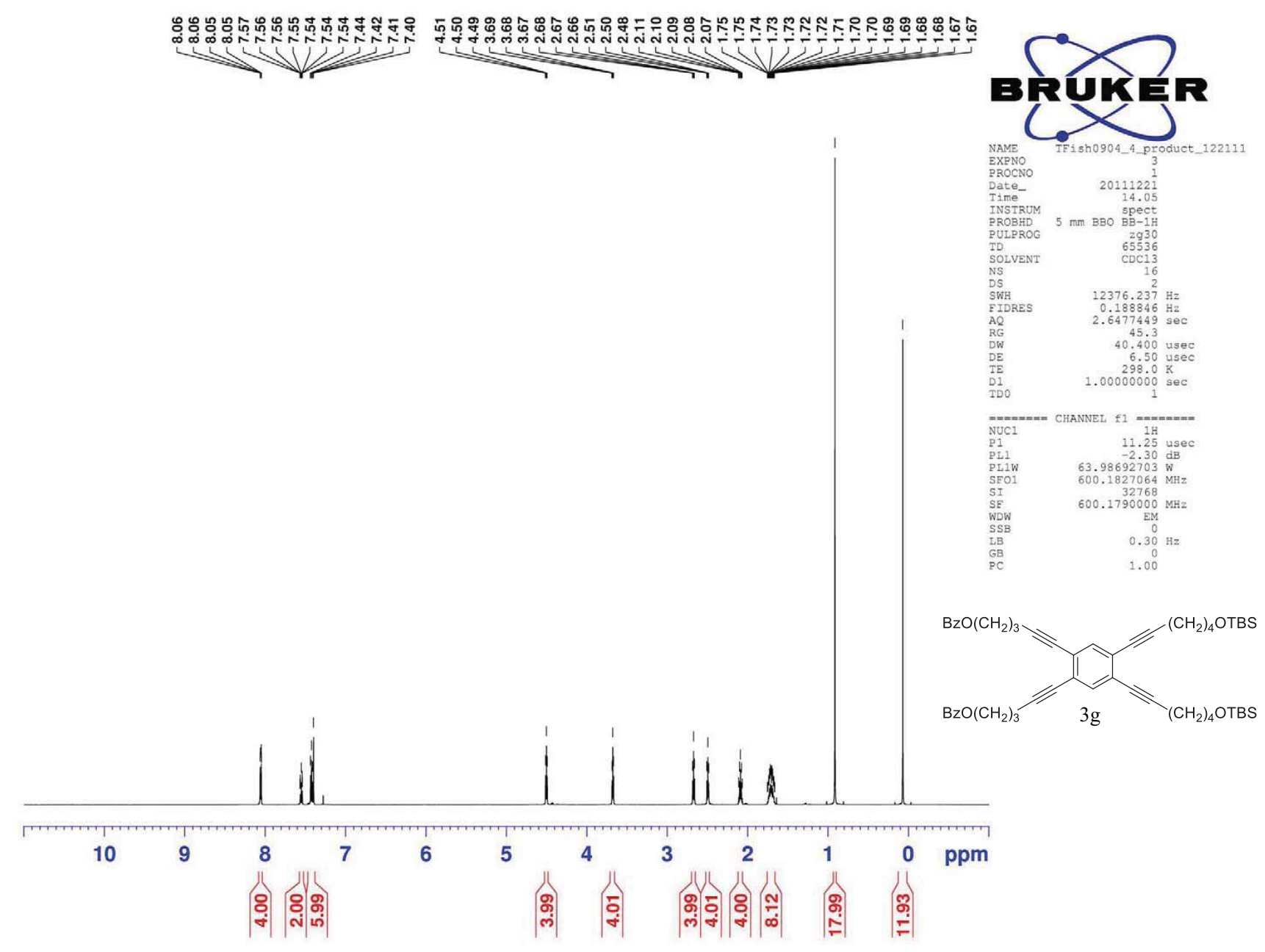




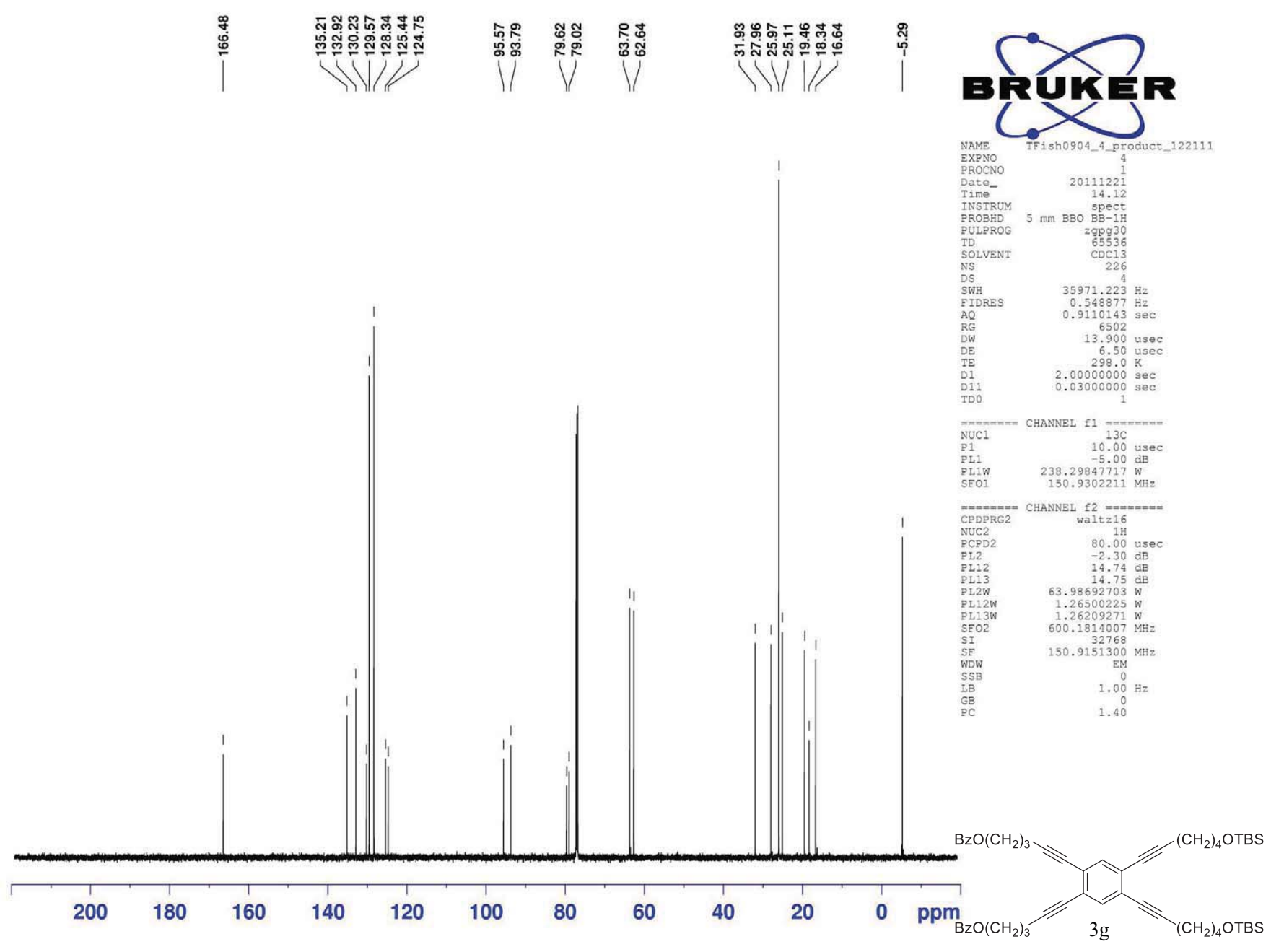




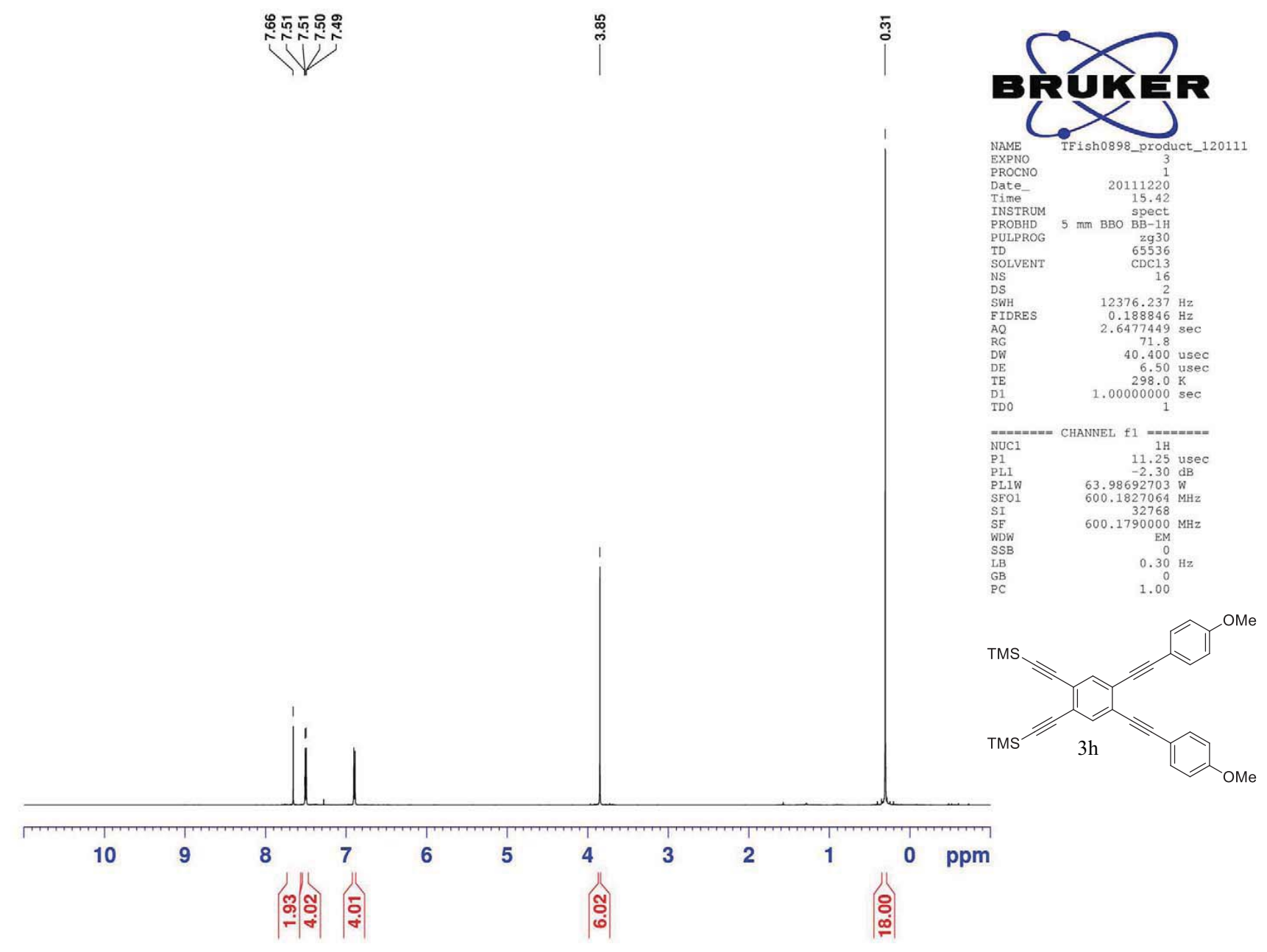




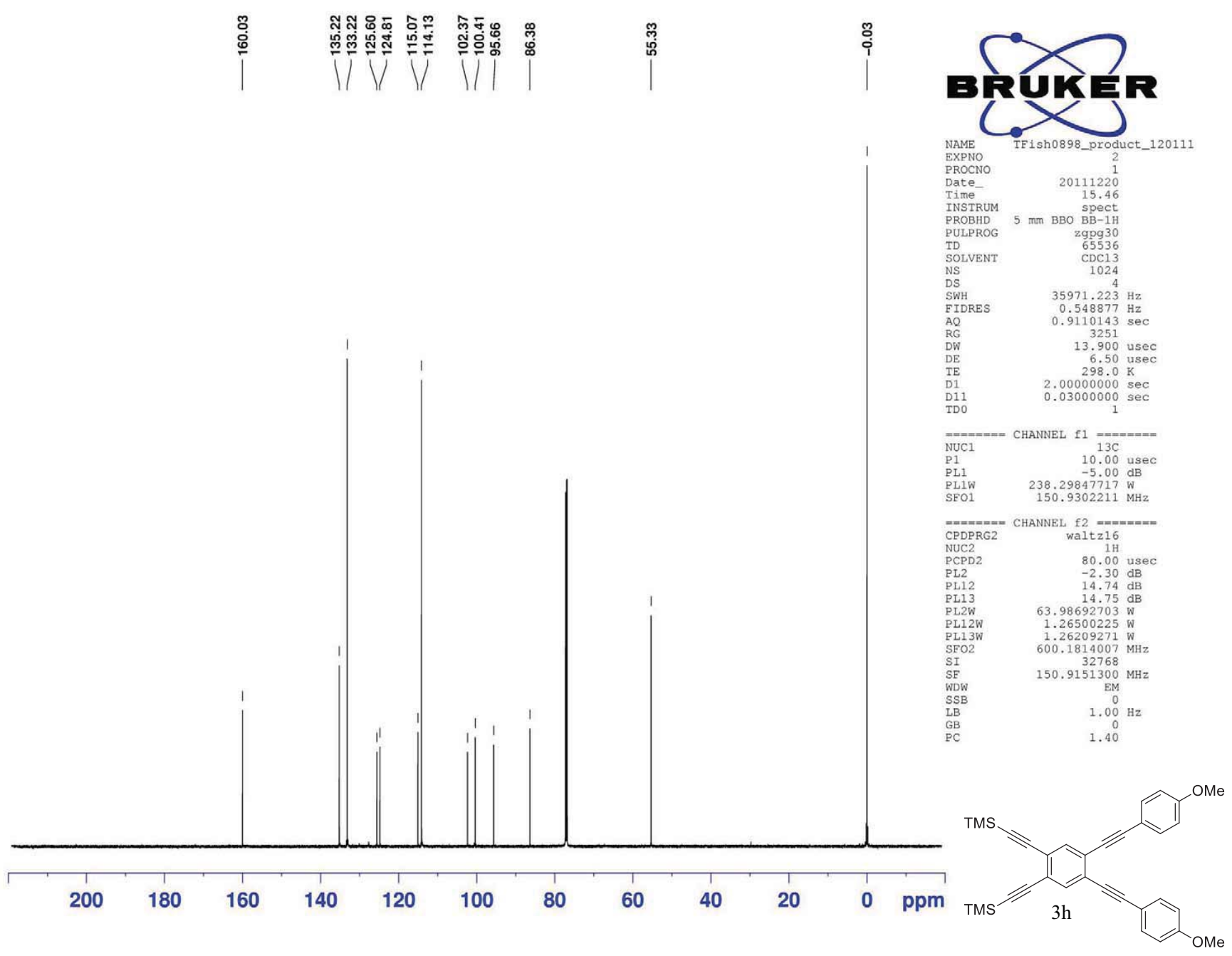




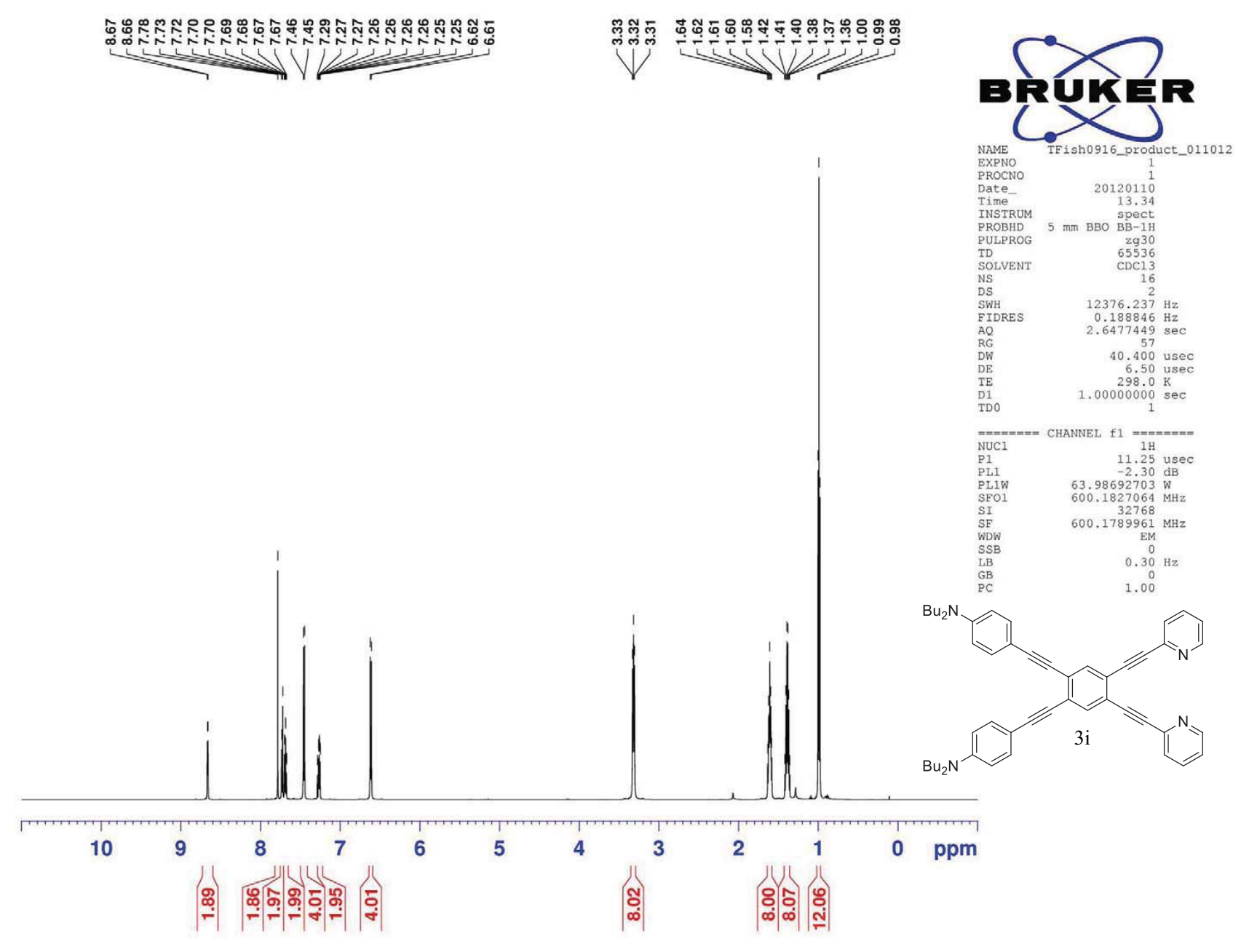




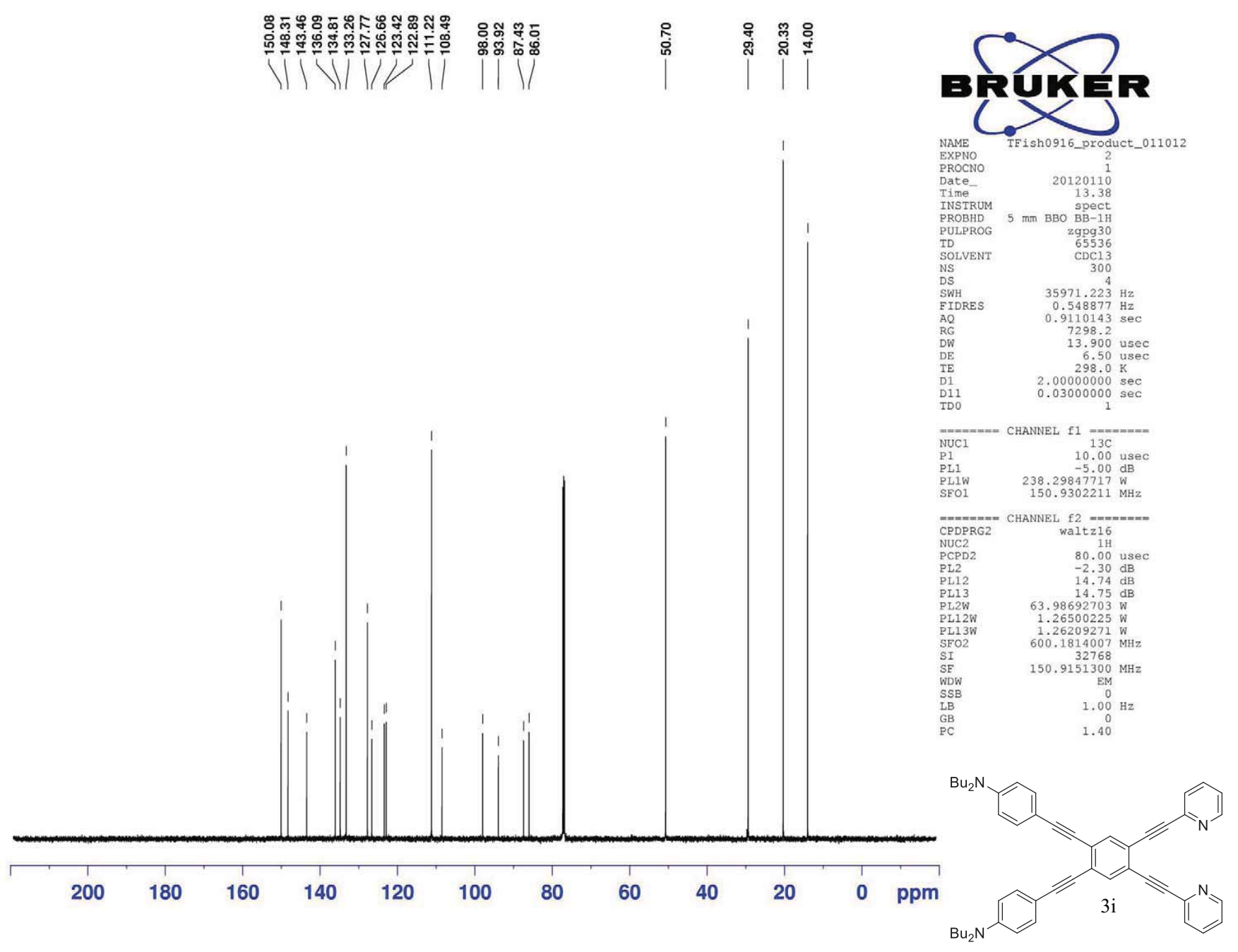

